\title{
Connection "Stripes" in the Primate Insula
}

\author{
Krockenberger M. ${ }^{1,2}$, Saleh T.O. ${ }^{1,2}$, Logothetis N.K. ${ }^{1,3}$ and Evrard H.C. ${ }^{1,2,4}$ \\ ${ }^{1}$ Werner Reichardt Center for Integrative Neuroscience, Karl Eberhard University of Tübingen, Tübingen, Germany \\ ${ }^{2}$ Max Planck Institute for Biological Cybernetics, Tübingen, Germany \\ ${ }^{3}$ Centre for Imaging Sciences, Biomedical Imaging Institute, University of Manchester, Manchester, UK \\ ${ }^{4}$ Translational Neuroscience Laboratories Division, Center for Biomedical Imaging and Neuromodulation (C-BIN), \\ Nathan Kline Institute for Psychiatric Research, Orangeburg, NY, USA
}

\section{Corresponding author:}

Henry C. Evrard

Translational Neuroscience Laboratories Division, Center for Biomedical Imaging and Neuromodulation (C-BIN), Nathan Kline Institute for Psychiatric Research, 140 Old Orangeburg Road, Orangeburg, NY 10962, USA, henry.evrard@nki.rfmh.org

Department Physiology of Cognitive Processes, Max Planck Institute for Biological Cybernetics, MaxPlanck-Ring 11, 72076, Tübingen, Germany, henry.evrard@ tuebingen.mpg.de

Functional and Comparative Neuroanatomy Group, Werner Reichardt Center for Integrative Neuroscience, Otfried-Müller-Strasse 25, 72076, Tübingen, Germany, henry.evrard@ cin.uni-tuebingen.de

\section{Running title:}

Primate insular stripes 


\begin{abstract}
The insula has been classically divided into vast granular, dysgranular and agranular sectors. Over the years, several distinct studies proposed subdivisions of these sectors, with however no consensus. We recently proposed a cyto- and myelo-architectonic partition in which each sector contained sharply delimited areas (Evrard et al. 2014 J Comp Neurol 522: 64-97). Some of these areas were further divided into distinct subareas with obvious functional implications. Here, we examined the spatial relationship between architectonic boundaries and tract-tracing labeling in the insula in the macaque monkey. Injections of neuronal tracers in distinct areas of the prefrontal or anterior cingulate cortices produced heterogeneous and discontinuous patterns of anterograde and retrograde labeling in the insula. These patterns were made of sharply delimited patches forming anteroposterior stripes across consecutive coronal sections. While the overall pattern of labeling varied with the injection site, the patches systematically coincided with specific architectonic subareas, particularly in the dysgranular insula. This unequivocally validates our prior architectonic partition and strongly supports the idea of a refined modular Bauplan of the primate insula. This modular organization may underlie a serial stream of integration of interoception with 'self-agency' and 'social' activities across distinct insulo-prefrontal processing units that need to be explored.
\end{abstract}




\section{Introduction}

The insular cortex constitutes the fifth lobe of the cerebral cortex in anthropoid primate (Reil 1809). Long perceived as a rather primitive and poorly organized limbic cortex, it recently reemerged as having a crucial role in representing the physiological state of the organs and tissues of the body (Craig 2002), and in contributing to emotional embodiment, with a role in shaping cognitive processes including perhaps subjective perceptual awareness (Craig 2009; Gu et al. 2013; Salomon et al. 2016). These functions are of paramount importance in human mental health and both the insula is, together with the cingulate cortex, one of the major target common to several distinct neuropsychiatric disorders (Goodkind et al. 2015). Thus, understanding how the insula is anatomically and functional organized is critically needed.

Beyond the basic macro-morphological of gyri and sulci (Reil 1809; Naidich et al. 2004), the primary approach to assess the organization of a brain region is to examine its histological architecture (Brodmann 1909; Vogt and Vogt 1919). The insular cortex has been classically divided into vast cytoarchitectonic sectors with, over the years, various proposals for further partitioning (Fig. 1; Brodmann 1909; Vogt 1911; Rose 1928; Brockhaus 1940; Mesulam and Mufson 1982; Kurth, Eickhoff, et al. 2010; Gallay et al. 2012; Evrard et al. 2014). Brodmann (1909) divided the insula into a large anterior agranular sector and posterior granular sector (Fig. 1A). He however stated that further examination should be carried on. Recognizing a different orthogonal orientation, the Vogt's (1911) recognized a dorsal granular sector and a ventral agranular sector that they further divided into myelo-architectonic areas aligned in some cases with the localization of the sulci (Fig. 1B). Rose (1928) and Brockhaus (1940) proposed much finer parcellations into multiple areas with rather abrupt cytoarchitectonic borders. They also added an intermediate dysgranular (or "mesocortical" with Brockhaus, and "propeagranularis" with Rose) sector in between the agranular and granular sectors already reported by Brodmann (Fig. 1C and D). Although Rose used only one human insula and did not represent the position of the sulci, taken together with the six insulae used by Brockhaus, their work suggested a parcellation into numerous (26 for Brockhaus to 31 for Rose) relatively sharply defined areas that readily suggested a compartmented functional organization. Later in the century, after a resurgence of interest for the insular cortex driven by new tract-tracing, lesion and recording studies primarily in non-human primates, a series of authors opted for a simpler parcellation recognizing only the three original sectors (Roberts and Akert 1963; Sanides 1968; Burton and Jones 1976; Mesulam and Mufson 
bioRxiv preprint doi: https://doi.org/10.1101/2020.11.03.361055; this version posted November 4, 2020. The copyright holder for this preprint

(which was not certified by peer review) is the author/funder. All rights reserved. No reuse allowed without permission.

1982) (e.g., Fig. 1F). In their seminal tract-tracing studies in the macaque monkey, Mesulam and

A

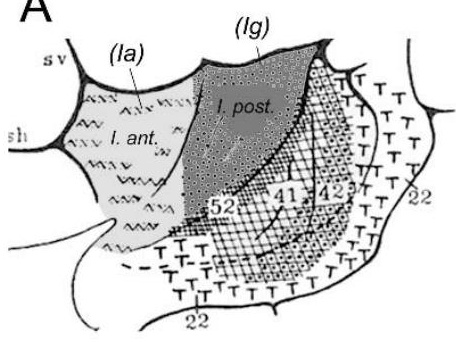

B
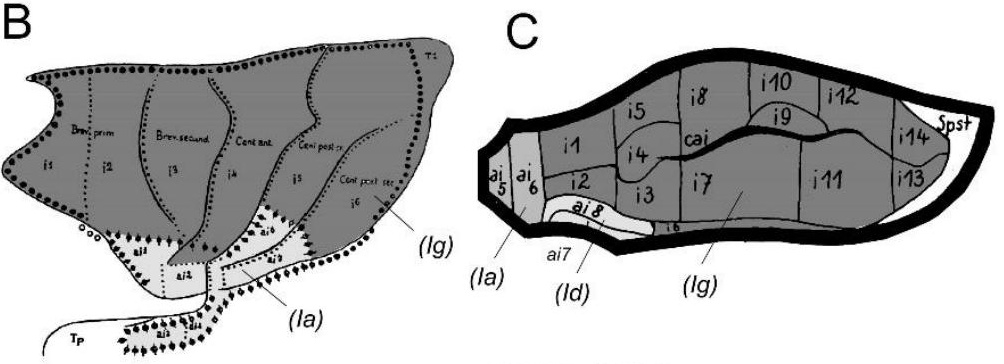

E

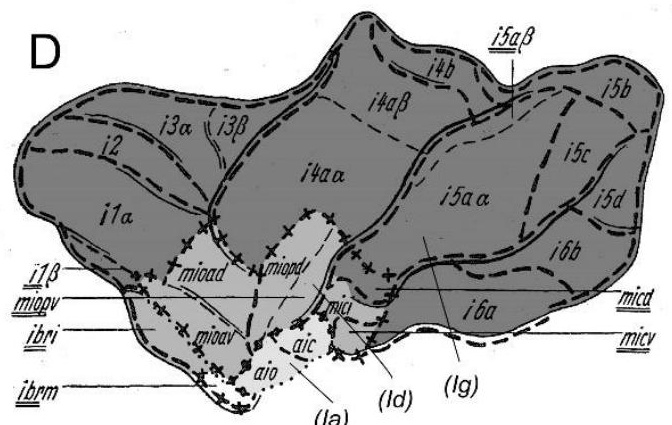

(la)

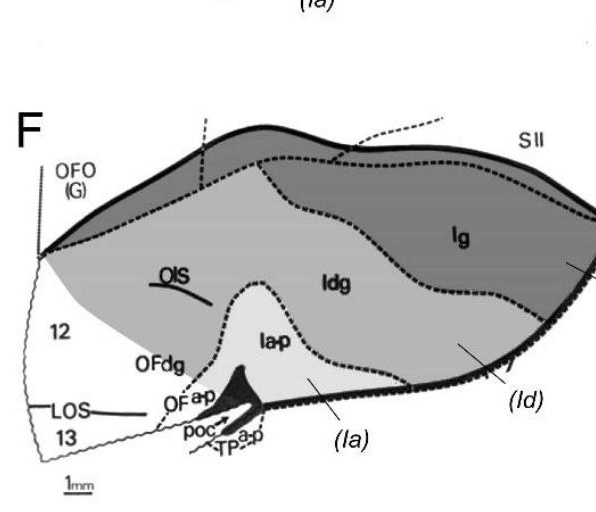

(d)

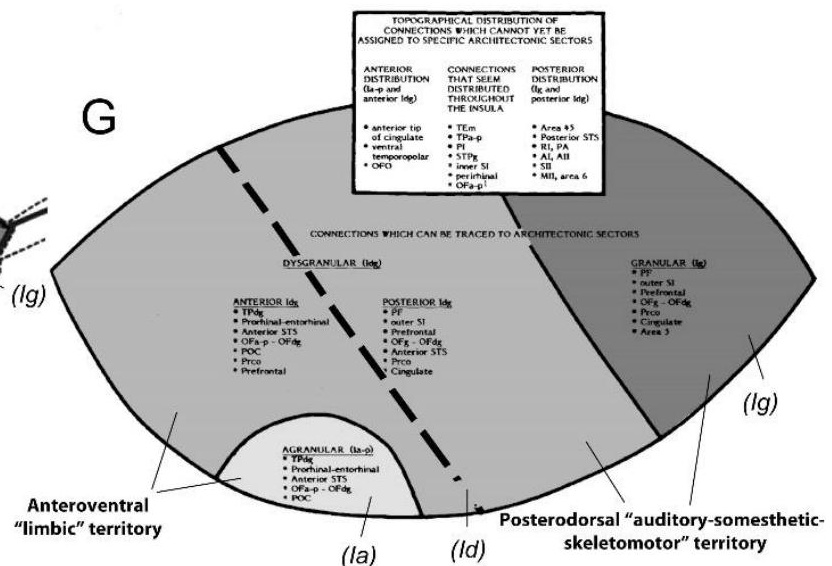

$\mathrm{H}$
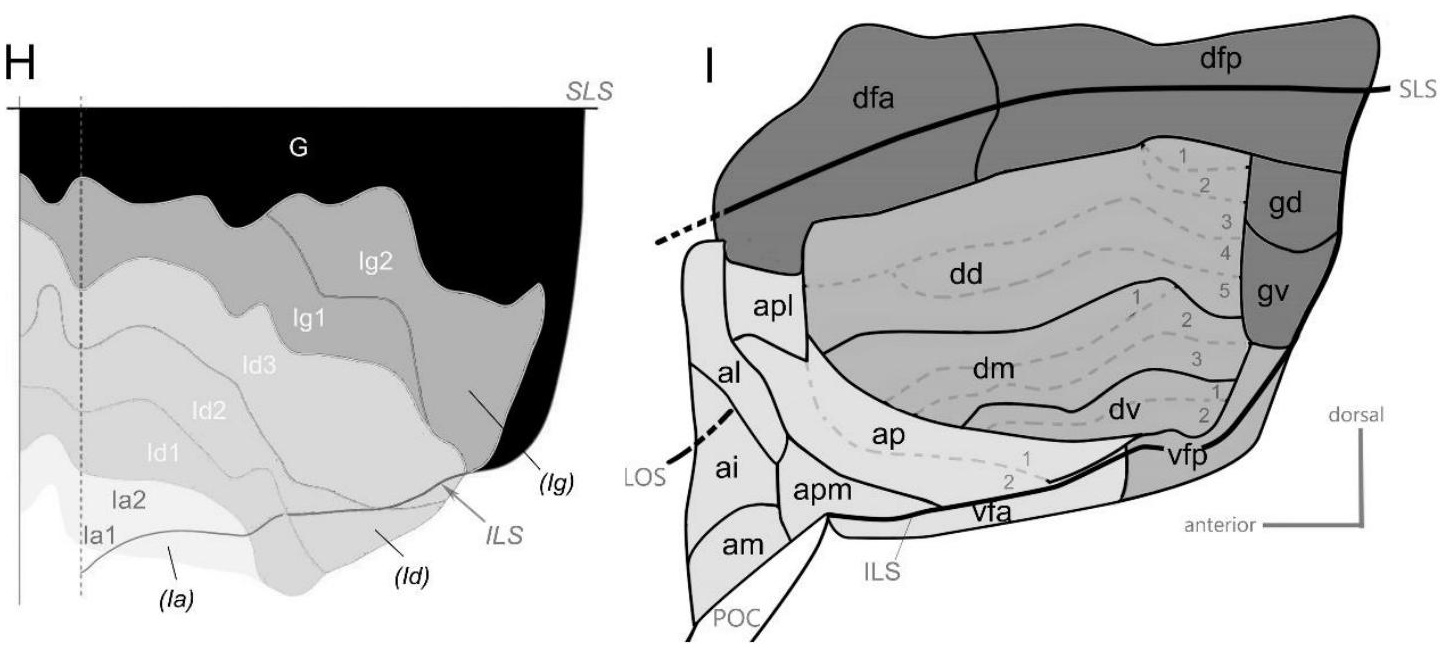
Figure 1. Flat maps drawings of the insular cortex showing the delineation of distinct sectors, areas or sub-areas in seven different cyto- and/or myelo-architectonic studies in humans or cercopithecine monkeys. A. Cytoarchitectonic division of the human insula into one anterior agranular (I. ant.; Ia) and one posterior granular (I. post.; Ig) sector (adapted with permission from Brodmann 1909). B. Myeloarchitectonic division of the human insula in which six myeloarchitectonic fields (i1-i6) were also cytoarchitectonically isocortical (or granular; Ig) and seven others (ail-i7) allocortical (or agranular; Ia) (adapted with permission from Vogt 1911). C. Cytoarchitectonic division of the hamadryas baboon (Papio hamadryas) insula into two agranular (ai7 and ai8; Ia), two dysgranular (ai5 and ai6; Id), and fourteen granular areas (i1-14; Ig) (adapted from Rose 1928). Rose's agranular and dysgranular sectors contain two (ail and ai10) and seven (ai2-6, ai9) additional agranular and dysgranular areas, respectively, that are not shown in this figure. D. Cyto-and myelo-architectonic division of the human insula into two allocortical (agranular; Ia), eight mesocortical (dysgranular; Id) and 16 isocortical (granular; Ig) areas (adapted from Brockhaus 1940). E. Cytoarchitectonic division of the human insula posterior to central sulcus, with a subdivision of the granular and dysgranular insula into two and three areas, respectively (adapted with permission from Kurth, Eickhoff, et al. 2010). F. Cytoarchitectonic division of the rhesus macaque (Macaca mulata) insula into one agranular (Ia), one dysgranular (Id) and one granular (Ig) sector (adapted from Mesulam and Mufson 1982). G. A similar view of the cytoarchitectonic map shown in panel E, overlaid with a hodological division into one anteroventral "limbic" territory and one posterodorsal "auditory-somesthetic-skeletomotor" territory (adapted with permission from Mesulam and Mufson 1982). The separation between the two territories crosses the middle of the dysgranular sector (thick dashed line in Id). H. Cytoarchitectonic division of the macaque insula into one vast "hypergranular" sector $(G)$, two granular areas (Ig1 and 2), three dysgranular areas (Id1, 2 and 3) and two agranular areas (Ial and 2) (adapted with permission from Gallay et al. 2012). Note that the parcellation of the insula in the same subject (Mk4) but using other histological or immunohistochemical stains did not coincide with the cytoarchitectonic areas and with each other (see Gallay et al. 2012). I. Cyto- and myelo-architectonic subdivision of the three classical sectors of the macaque insula into four granular areas (Idfa, Idfp, Igd, Igv), four dysgranular areas (Idd, Idm, Idv, Ivfp), and seven agranular areas (Ial, Iai, Iam, Iapl, Iap, Iapm, Ivfa) (adapted from Evrard et al. 2014). Three of the dysgranular areas (Idd, Idm and Idv) and one of the agranular areas (Iap) were further subdivided in 2 to 5 subareas. In all panels, top is dorsal and left is anterior. The different degrees of cytoarchitectonic granularity are represented with different tones of gray (black, "hypergranular"; dark gray, granular; middle gray, dysgranular; light gray, agranular). For the abbreviations in A to $G$, see original publications. For the abbreviations in $H$, see the present abbreviations list. (adapted with permission from Evrard et al., 2014).

Mufson divided the insula into two vast territories, with a vague gradual separation crossing the middle of the dysgranular sector, with no recognition of a finer internal organization (Fig. 1G) (Mesulam and Mufson 1982; Mufson and Mesulam 1982; Mesulam and Mufson 1985; Mufson et al. 1997). The interest for the insula sparked again with new findings in functional imaging in humans and with the proposal of a crucial role of the insula interoception (Craig 2002). Using perikaryal stain with a well-established "observer-independent" and probabilistic parcellation method (Amunts et al. 2002), Kurth and colleagues map the human insula posterior to its central sulcus (Kurth, Eickhoff, et al. 2010). They proposed a consistent division of the granular and dysgranular sectors into two and three sharply-delimited areas, respectively (Fig. 1E). Using multiple histological and immunohistochemical stains in macaque monkeys and humans, Morel, Gallay and colleagues also recognized a finer division of the classical sectors (e.g., Fig. 1H) (Gallay et al. 2012; Morel et al. 2013). However, they did not observe any consistent coincidence between the borders defined by the different stains. Finally, using cyto- and myelo-architectonics in 20 cynomolgus macaque monkeys, Evrard and collaborators reported the parcellation of the classical granular, dysgranular and granular sectors into respectively 4, 4 and 7 sharply delimited 
and highly consistent areas (Evrard et al. 2014). Three dysgranular areas and one agranular area were further subdivided into subtle sub-areas with optimal coincidence of the boundaries defined by the cyto- and myelo-architectures. This new map was relatively consistent with the map of the posterior insula by Kurth et al. (2010) and with map of the anterior agranular insula, anterior to the limen, by Carmichael and Price (1994) where cross-stain coincidence was also reported. (For an exhaustive comparison of these different maps, see the Discussion in Evrard et al., 2014.)

Cortical areas can be hard to define and identify, and their exact number in any species is uncertain (Kaas 2012). As expressed in our prior report, architectonic parcellation is biased by the subjective judgment of the observer and complicated by the inter-individual variability typically encountered across macaque brains (Evrard et al. 2014). In our architectonic examination, we consistently observed a thinner parcellation of the large dysgranular areas into narrow longitudinal sub-areas (Fig. 1H). However, these sub-areas were difficult to demonstrate to others in photomicrographs and were thus presented as "suggestions". Neuronal tract-tracing adds an unbiased criterion for the parcellation of the cerebral cortex. The demonstration of an overlap between our cyto-/myelo-architectonic sub-areas and patterns of anterograde and/or retrograde labeling would provide an ideal demonstration of the validity of our insular parcellation. Therefore, in the present study, we examined the distribution of the labeling produced in the insula of the macaque monkey with injections of tracers in the prefrontal and cingulate cortices. We compared the distribution of the labeling with the architectonic parcellation defined in our prior study. We observed an optimal coincidence of architectonic and hodological boundaries.

\section{Material and Methods}

\section{Tracer injection and histological processing}

The present data was obtained from five adult rhesus macaque monkeys (Macaca mulata) of either sex. These monkeys were injected with neuronal tracers in the context of separate studies of the connectivity of the prefrontal cortex in the laboratory of Prof. Dr. Joseph L. Price at the Washington University of Saint-Louis, Missouri, USA. All animal protocols were approved by the Animal Studies Committee of Washington University and they complied with the guidelines of the NIH for the care and use of laboratory animals.

Detailed descriptions of the procedures for the microinjection of tracers, tissue fixation and histological processing have been previously published (e.g., Carmichael and Price 1994; Ferry et 
al. 2000). Briefly, the tracer injections were made under deep general anesthesia and aseptic surgical conditions using glass micropipettes positioned through craniotomies at specific stereotaxic coordinates to reach selected cortical targets. The anesthesia was induced with ketamine (10 mg/kg, i.m.) and xyalzine $(0.67 \mathrm{mg} / \mathrm{kg}$, i.m.) and maintained with a gaseous inhalation of a mixture of oxygen, nitrous oxide, and halothane. A long-lasting analgesic (buprenorphine; $0.1 \mathrm{mg} / \mathrm{kg}$, i.m.) was injected after the surgery as the animal recovered from the anesthesia. The tracers included biotinylated dextran amine (BDA), lucifer yellow (LY) and fluororuby (FR), at $5 \%$ or $10 \%$ in a $0.001 \mathrm{M}$ phosphate buffered saline (pH 7.4) (PBS). The volumes injected varied between 0.3 and $1.2 \mu \mathrm{L}$.

After a survival period of $\sim 14$ days, the animals were sedated with ketamine (10 mg/kg, i.m.), deeply anesthetized with sodium pentobarbital (30 mg/kg, i.p. or i.m.) and transcardially perfused with PBS, followed by a sequence of $4 \%$ paraformaldehyde fixative phosphate buffers (1 liter of $4 \%$ paraformaldehyde, $\mathrm{pH} 6.5,2$ liters of $4 \%$ paraformaldehyde and $0.05 \%$ glutaraldehyde, $\mathrm{pH}$ 9.5, and finally 1 liter of the same solution with $10 \%$ sucrose). The brains were removed, blocked in the coronal plane and post-fixed in a $30 \%$ sucrose PBS at $4^{\circ} \mathrm{C}$. After 2 to 6 days, the blocks of the brain were frozen and alternating 1-in-10 series of 50- $\mu$ m-thick coronal sections were made using a sliding microtome, collected in PBS, and processed for histology or immunohistochemistry. BDA, LY and FR were revealed in separate series using the avidin-biotinperoxidase staining method (Carmichael and Price 1995). One additional series was stained with the Nissl method for neuronal cell bodies and, in two cases, one series was stained with the Gallyas method for myelin (Carmichael and Price 1994).

\section{Data analysis}

In a first analysis session, the location of the injection site in the prefrontal or cingulate cortex, and of the anterograde and/or retrograde labeling in all serial sections of the insular cortex ipsilateral to the injection site were examined at $5 \mathrm{x}, 10 \mathrm{x}, 20 \mathrm{x}$ and/or $40 \mathrm{x}$ using an upright brightfield microscope (Olympus $\mathrm{BH} 2$ ) and manually plotted using optical encoders attached to the microscope stage and interfaced with a personal computer (Minnesota Datametrics, St. Paul, MN, USA). This plotting system allowed a precise mapping of the location of retrogradely labeled cell bodies and an approximate, general representation of the location and density of the anterograde labeling. Composite of consecutive coronal maps of the labeling overlaid on maps of 
structural landmarks (cortical gray matter, delineation of cortical granular layer 4, claustrum...) were assembled in Adobe Photoshop (San Jose, CA, USA) and used to generate flat map representations (see below) of the overall distribution of the anterograde and retrograde labeling in the insula.

In a second analysis session, one to four representative series of coronal sections of the insula were selected in each case for a more precise mapping of the anterograde (and retrograde) labeling. These sections were photomicrographed at high resolution $(0.2 \times 0.2 \times 1$ microns, $21 \mathrm{z}$-stack levels $)$ using the 10x and 20x planapochromatic objectives of a motorized microscope slide scanner (AxioScan.Z1, Carl Zeiss, Göttingen, Germany). The virtual sections were uploaded in a custommade anatomical drawing software. The contour of the insula and its main anatomical landmarks (grey/white matter edge, layer 4, and claustrum) were drawn and the exact position of each retrogradely labeled neurons and anterogradely labeled axon terminal was plotted at high magnification while scrolling through the twenty-one 1- $\mu$ m-tick focal points of the $\mathrm{z}$-stack of the virtual sections to confirm the identity of the objects (i.e., stained cell body or axon terminal) (see Results).

In a third and independent analysis session, the cytoarchitecture and, when available, myeloarchitecture of the insula (Nissl- and Gallyas-stained sections) was examined by at least 2 independent examiners (MK, HCS and/or TOS) using both a stereomicroscope and the virtual sections. The areal and modular boundaries were drawn using architectonic criteria defined in prior studies (Carmichael and Price 1994; Evrard et al. 2014) (Fig. 1H). The plots of labeling and the corresponding architectonic maps were aligned together and figures were prepared in Adobe Photoshop (San Jose, CA, USA) without alteration other than to reach matching levels of brightness in all the photomicrographs. Finally, a lateral flat map view of the insula comparing the architecture and distribution of the anterograde/retrograde labeling was prepared for each case, using a procedure described in detail elsewhere (Evrard et al. 2014; see also Results).

\section{Results}

\section{General observations}


Krockenberger et al.: Primate insular stripes

Figures 2A-E show drawings of the tracer injection sites in coronal sections from the five cases reported in this contribution (from A to E: OM85, OM42, OM27, OM30 and OM69). With the exception of OM85, these injection sites were described in previous articles (Ferry et al. 2000). All injections produced a dense core of tracer accumulation (black shapes in Fig. 2) and, in some cases, a diffuse halo of tracer diffusion surrounding the core (dark gray shapes in Fig. 2). The injection of LY in OM85
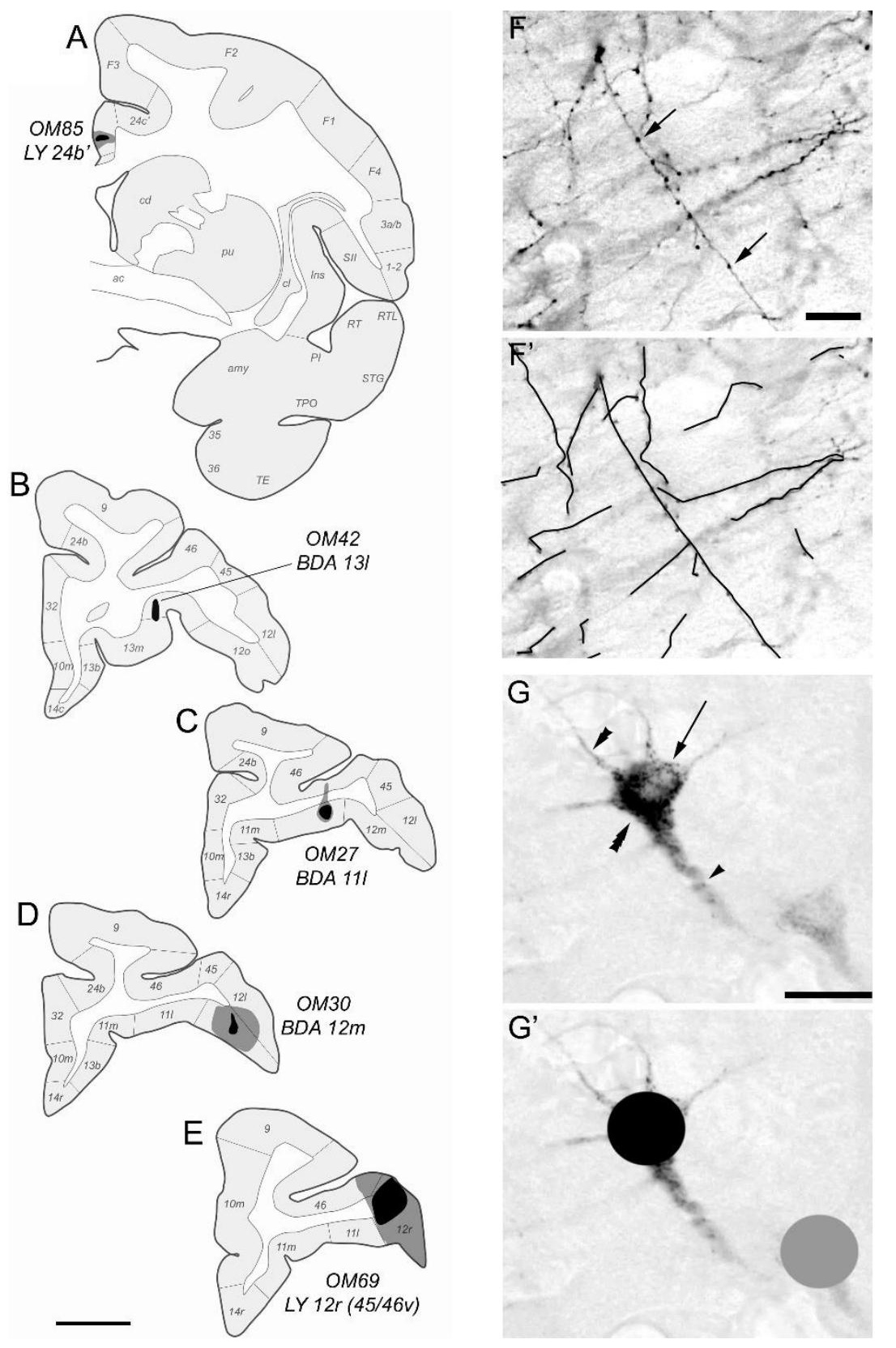

Figure 2. A-E. Drawings of the sites of injection of neuronal tracers in coronal sections of the right hemisphere of the cerebral cortex in five rhesus macaque monkeys. Each injection site is represented by a black core surrounded, in some cases, by a gray halo of diffusion. The five different cases are laid out from the most posterior (OM85) to the most anterior (OM69) injection. A. Injection site of lucifer yellow $(L Y)$ in area 24b' in OM85. B. Injection site of biotin dextran amine (BDA) in area $13 l$ in OM42. C. Injection site of BDA in area 111 in case OM27. D. Injection site of BDA in area $12 \mathrm{~m}$ in case OM30. E. Injection of $L Y$ in area12r (and 45/46v, not shown) in OM69. Medial is left; dorsal is top. Scale bar $=5 \mathrm{~mm}$. See the abbreviation list. $\boldsymbol{F}$. Photomicrograph of representative retrogradely labeled pyramidal neurons in the insular cortex in case OM85 with an injection of lucifer yellow in area 24b'. The triple, double and single arrowheads point to the labeled perikarya, one of the basal dendrites and the apical dendrite, respectively. The arrow points to the unlabeled cell nucleus. $\boldsymbol{G}$. Photomicrograph of representative anterogradely labeled axon terminals in the insular cortex in case OM85. The arrows point to examples of labeled varicosities localized along the axon fiber. $\boldsymbol{F}^{\prime}-\boldsymbol{G}^{\prime}$. Illustration of the labeling charting procedure. Scale bar $=10 \mu \mathrm{m}$. 
spread across all cortical layers within a small portion of the cingulate area 24b' (Fig. 2A). The injection of BDA in OM42 reached the medial portion of the orbital prefrontal area 131 with a negligible spread to area 13m (Fig. 2B) (Ferry et al. 2000). The injection of BDA in OM27 was confined to a small portion of the orbital prefrontal area 111 with a thin halo spreading along the pipette track in area 46 (Fig. 2C) (An et al. 1998). The injection of BDA in OM30 filled a rather large portion of the orbital prefrontal area $12 \mathrm{~m}$ with a small spread of the halo to area 121 (Fig. 2D) (An et al. 1998). The injection of LY in OM69 filled a rather large portion of the lateral prefrontal area 12r (Fig. 2E) and spread posteriorly in small portions of the dorsolateral prefrontal areas 45 and 46 (not shown) (Saleem et al. 2008).

All five injections produced anterograde and/or retrograde labeling in the insula. Figures $2 \mathrm{~F}$ and $\mathrm{G}$ show high magnification photomicrographs of representative anterograde and retrograde labeling, respectively. Anterogradely labeled axon terminals were recognized by darkly stained cortico-cortical axonal fibers bearing varicosities (presumably synaptic boutons), identified as small beaded structures along the stained fibers (Fig. 2H). Each clearly distinct individual fiber bearing varicosities was charted using a continuous line (Fig. 2H'). The retrograde labeling occurred in typical cortico-cortical projection pyramidal neurons. It was identified by the staining of intracellular granules (presumably endocytoplasmic vesicles) filling the triangular soma and the proximal portion of the apical and basal dendrites while leaving the cell nucleus unstained (Fig. 2G). Intensely and weakly labeled perikarya (see the two neurons labeled in Fig. 2G) were charted in maps of the insula using black and gray symbols, respectively (Fig. 2G').

Figures $3 \mathrm{D}$ to $\mathrm{H}$ show the flat map representations of the overall distribution of anterograde and/or retrograde labeling in the five cases. (Figures 3A-C illustrate the construction of these flat maps from series of consecutive coronal section drawings, using one section from OM85 as an example. See also Evrard et al. 2014.) The tracer injections made in distinct prefrontal or cingulate areas produced broadly distributed or rather restricted labeling, regardless of the size of the injection site. For example, the small injection of LY in area 24b' in OM85 produced much more insular labeling (Fig. 3D) than the larger injection of BDA in area 12m in OM30 (Fig. 3G) or LY in area 12r in OM69 (Fig. 3H). In all cases but one (i.e., OM30; Fig. 3G), the labeling occurred contiguously across several adjacent areas and sub-areas. For instance, in OM85, labeling was present in at least 11 consecutive architectonic entities throughout the dorso-ventral extent of the insula, with no 'gap' being entirely free of labeling (Fig. 3D). Yet, as illustrated in the figure and 
as will be demonstrated in the next section, the density (and to some extent layer distribution) of the labeling markedly and rather sharply varied throughout the insula, with a clear coincidence with the architectonic changes differentiating one cytoarchitectonic area or sub-area from the next.
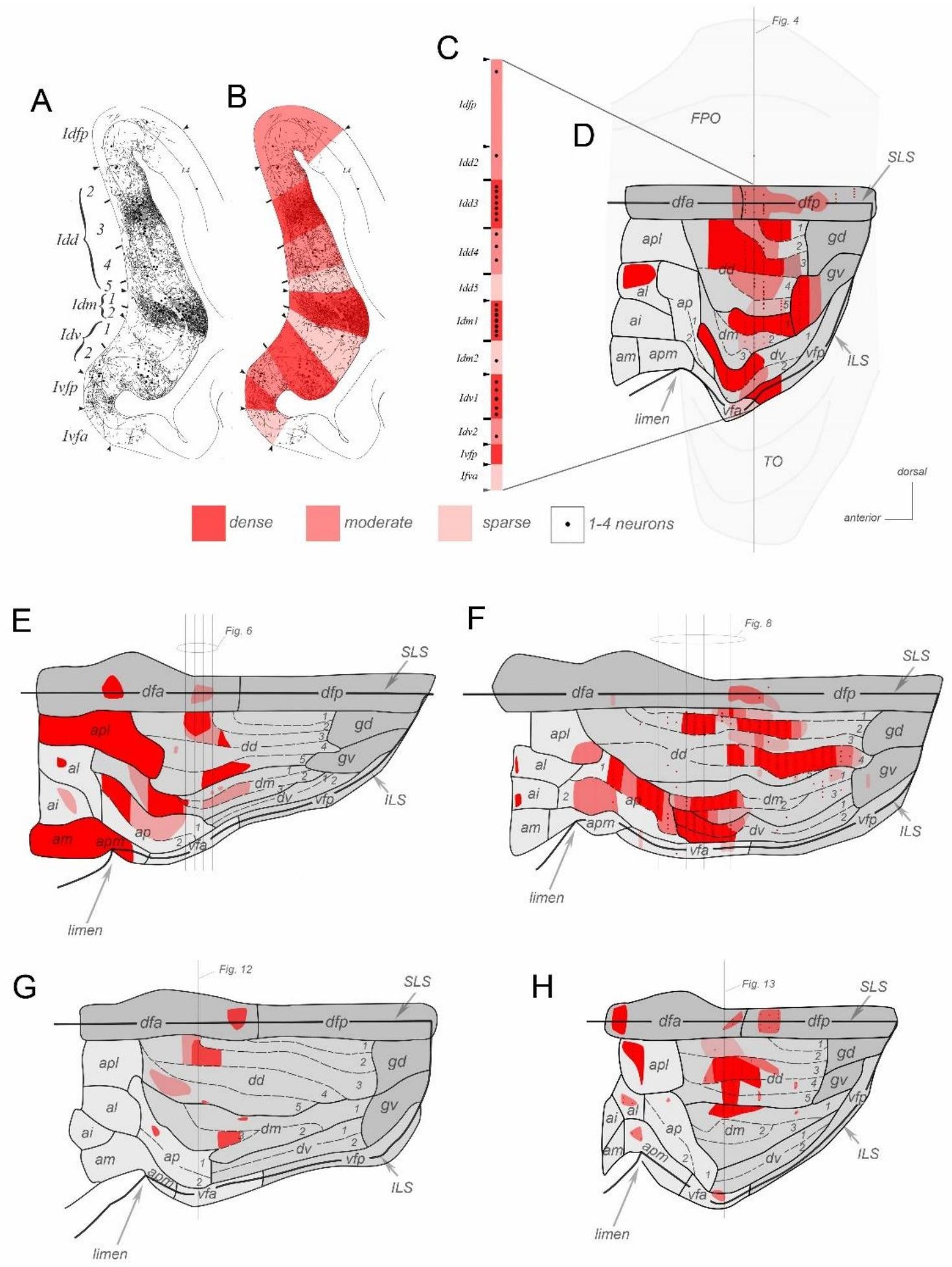
Figure 3. Flat map representations of the distribution of anterograde (shades of red) and retrograde (dark dots) labeling across the distinct architectonic areas and subareas of the insular cortex in five rhesus macaque monkeys with injections of tracers in different prefrontal or cingulate cortical areas. Panels A to $C$ illustrate the method used to re-create the flat map of the insula shown in panel $D$ for case OM85. The same principle was applied for all flat maps (Evrard et al. 2014). A. Original drawing of anterograde (thin lines) and retrograde (dots) labeling in one coronal section of the insular cortex in case OM85. B. Red-shades color-coding of three different densities of anterogradely labeled terminals (weak, moderate, dense). C. Transfer of the colorcoding and overall number of retrogradely labeled neurons onto a flattened representation of the areas and labeling of the section. $\mathbf{D}-\boldsymbol{H}$. Flat map representations of the distribution of anterograde and/or retrograde labeling in the insula in cases OM85 (D), OM42 (E), OM27 (F), OM30 (G) and OM69 (H). In panel D, the frontoparietal (FPO) and temporal (TO) opercula are represented with a pale gray. In panels $E-H$, the representation of FPO and TO was omitted to avoid cluttering the figure. In all flat maps, the thick lines represent the superior and inferior limiting sulci (SLS and ILS). In all cases, the coronal sections were aligned using SLS, which is therefore represented by the straight line. The thinner plain contours delineate architectonic areas. The dashed contours delineate subareas within an area. The vertical lines crossing the maps indicate the anteroposterior level of the coronal sections shown in Figures 4 to 13 to illustrate the overlap of architectonic and hodological entities (see text).

\section{Architecto-hodological overlap}

Figures 4 to 13 illustrate the overlap of tract-tracing labeling pattern variation with architectonic boundaries in representative coronal sections from each of the five cases. Figures 4, 6, 8, 12A-C and 13A-C show general low magnification views whereas Figures 5, 7, 9-11, 12D-E and 13E-F show corresponding high magnification demonstrations of the cyto- or myelo-architectonic boundaries. In all five cases, the pattern of anterograde and/or retrograde labeling was heterogeneous. The heterogeneity was characterized by isolated or multiple contiguous sharply delimited 'patches' of labeled cells and/or terminals. The metric and sharp delimitations of these patches were directly reminiscent of the architectonic sub-areas (or "modules") defined in our prior contribution (Evrard et al. 2014). In fact, as detailed below, each patch of labeling overlapped indeed with a distinct architectonic entity. In general, the cyto- and myelo-architectonic features of the sub-areas described below for each case were comparable to the generic features reported in our earlier report (Evrard et al. 2014). 
OM85. Labeling in OM85 occurred in a large middle portion of the insula, from Idfp to Ivfa, as well as in a small region within Ial (Fig. 3D). Figure 4A shows a representative low magnification photomicrograph of a coronal section from the middle portion of the insula, immunoreacted to visualize the anterograde and retrograde labeling produced by the injection of LY in area 24b'. Two conspicuous and sharply delimited patches of labeling are readily visible at this low magnification. Figure 4B shows the corresponding plot of individual retrogradely labeled perikarya and anterogradely labeled terminals. Figures $4 \mathrm{C}$ and 5 show the localization of cytoarchitectonic borders on an adjacent Nissl-stained section, at low and high magnifications, respectively. The same borders were transposed on the plot of anterograde and retrograde labeling (Fig. 4B).
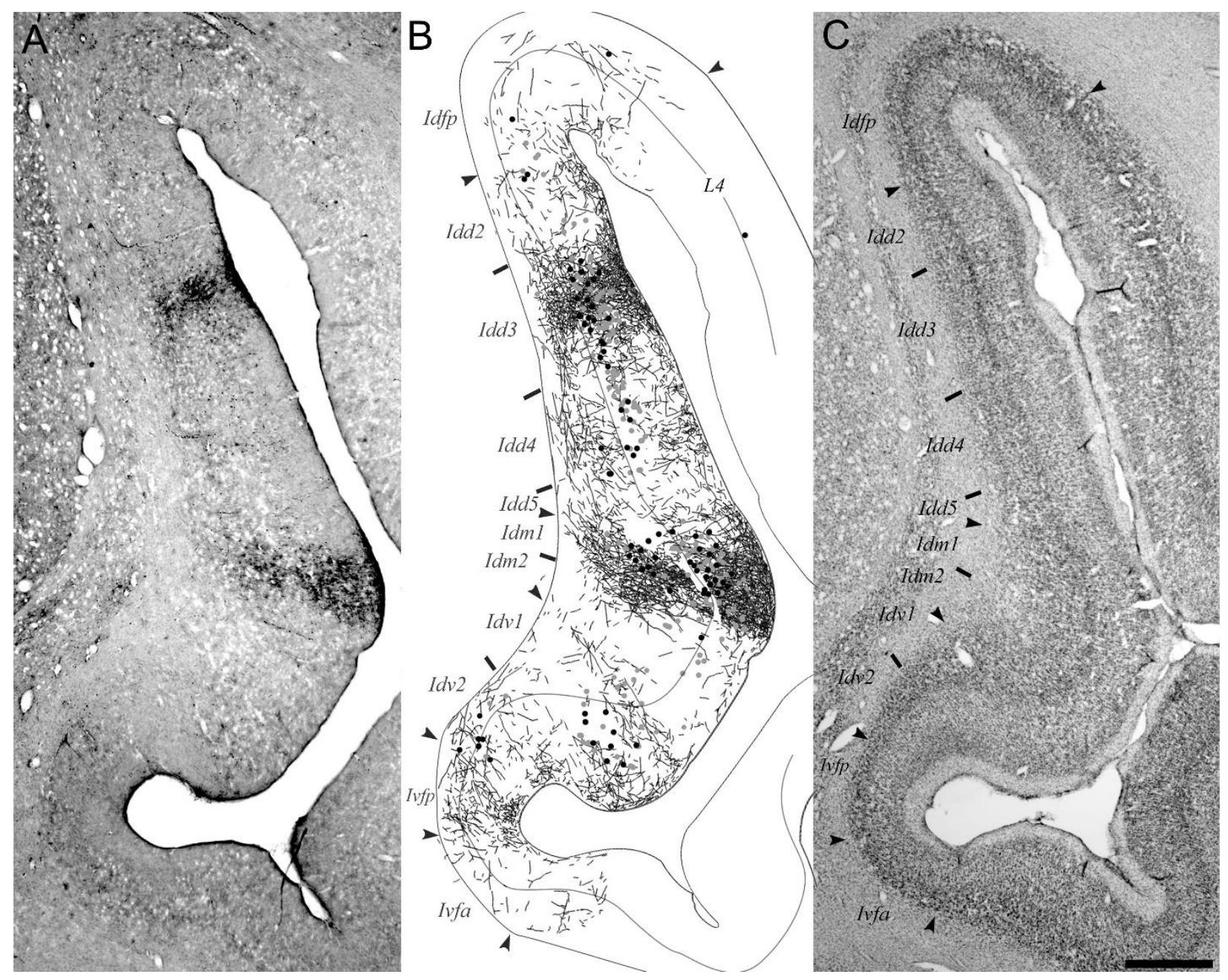
Figure 4. Composite showing the spatial overlap of patches of anterograde and retrograde labeling with specific cytoarchitectonic subareas of the insular cortex in case OM85 with an injection of lucifer yellow (LY) in cingulate area 24b'. A. Low magnification photomicrograph of a coronal section of the insula immunoreacted to visualize LY. Two patches of labeling are readily visible at low magnification. B. Plot of individual anterograde labeled terminals and retrogradely labeled perikarya made at high magnification in the same section as shown in panel A. Darkly and moderately stained perikarya are represented with black and gray dots, respectively. C. Low magnification photomicrograph of an adjacent Nissl-stained section showing the cytoarchitectonic boundaries between areas (arrowheads) and subareas (ticks). The same boundaries were transposed to the photomicrograph in panel B. Top is dorsal; left is medial. Scale $=1 \mathrm{~mm}$.

From dorsal to ventral, we observed two regions with moderate anterograde labeling that corresponded to Idfp and Idd2, with clearly more labeling in Idd2 than in Idfp (Fig. 4B). The architectonic border between Idfp and Idd 2 was characterized by a distinct vertical shift of the position of the slightly less discernable layer 4, a thickening and more collimated organization of layer 3 with barely discernible sub-layers $3 \mathrm{a}$ and $\mathrm{b}$, and a broader layer 5 in Idd 2 compared to Idfp (Fig. 4C and 5A). The next region of labeling corresponded to Idd 3 and presented one of the most obvious and edifying cases of overlap between a tract-tracing labeling 'patch' and an architectonic sub-area (Fig. 4A-C and 5A). Idd3 contained a large group of darkly and moderately retrogradely labeled neurons in layer $3 \mathrm{~b}$ and a dense mesh of anterogradely labeled fibers in layers 1 to 5. Architectonically, Idd3 was characterized by the presence of a thin but densely populated ('darker' in appearance) and very distinct layer 4 with particularly sharp delimitations (already distinct at low magnification) at the borders with Idd2 and Idd4, in which layer 4 was broader and less discernable (Fig. 4C and Fig. 5A). Idd3 had clearly separate and equally thick layers 3a and 3b, populated in this case with much 'darker' neurons than in Idd2. Layer 5 was slightly more compact and populated with smaller cells than in Idd2 and thinner than in Idd4. In contrast to Idd3, the moderate labeling in Idd4 was characterized by the presence of a thin 'band' of retrogradely labeled cells (contained within an equally thinner layer $3 \mathrm{~b}$ ), and distinctly less anterograde labeling, occurring mainly in outer and inner layers but not much in middle layers (Fig. 4B). Architectonically, in comparison to Idd3, Idd4 had a broader but less distinct layer 4, a broader layer 3 with a thin but poorly discernable layer $3 \mathrm{~b}$ and larger layer 3a, and broader layers 5-6 (Fig. 4C and 5A). Idd5 had sparse anterograde labeling and no retrograde labeling (Fig. 4B). It was architectonically characterized by a thinner and less populated (sparser) layer 3 and larger cells in layer 5 (Fig. 4C and Fig. 5B). 

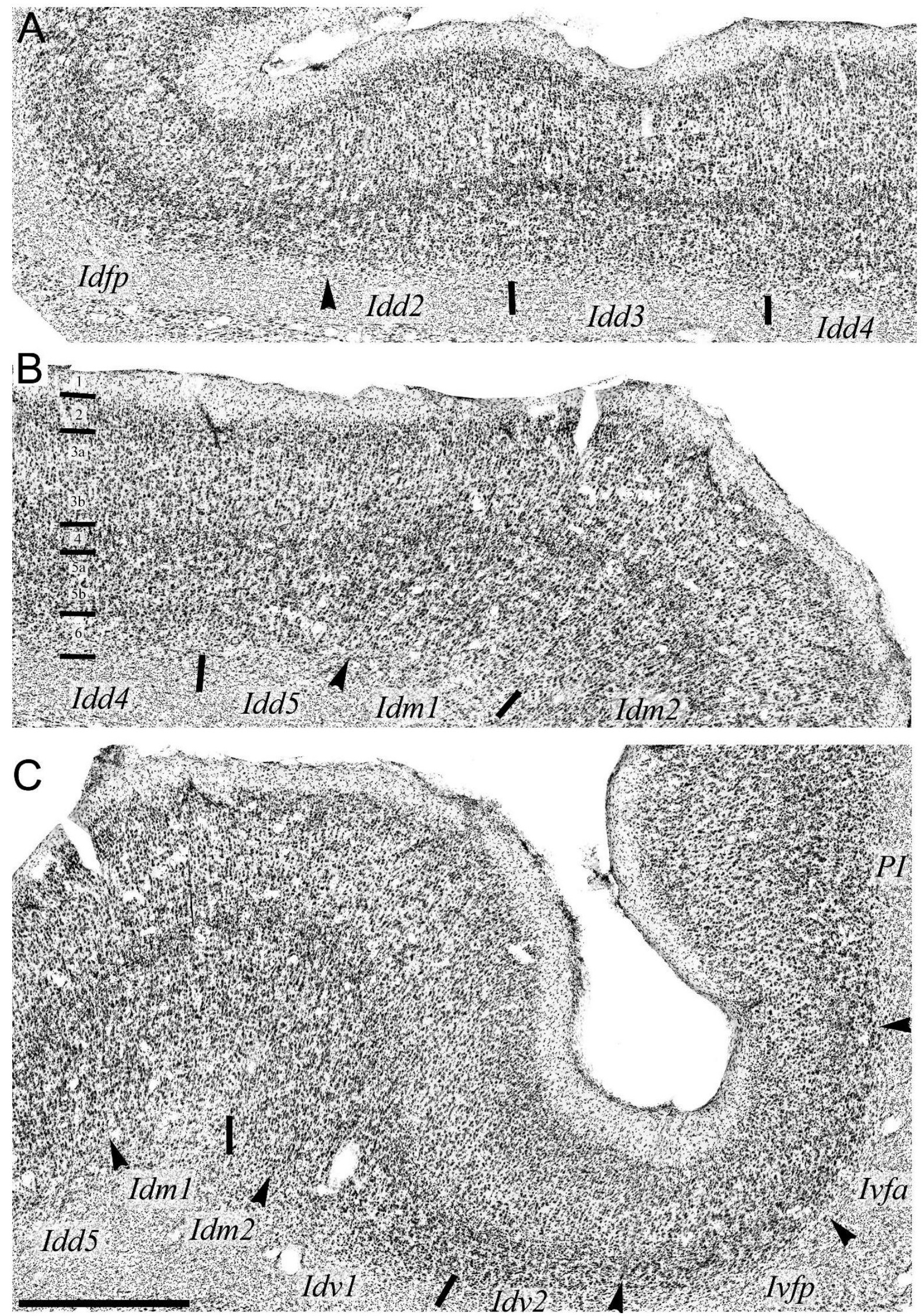
Krockenberger et al.: Primate insular stripes

Figure 5. High magnification photomicrographs of the (A) dorsal, (B) middle and (C) ventral parts of the Nissl-stained section of the insula shown in panel C of Figure 4 in case OM85. The arrowheads and ticks mark the cytoarchitectonic boundaries between areas and subareas, respectively. The horizontal tick marks in panel B mark the separation of the cortical layers. Top is medial and left is dorsal. Scale bar $=1 \mathrm{~mm}$.

Idm1 contained again a large population of retrogradely labeled neurons located this time both in layers 3 and 5, which differed from Idd3 in which labeled neurons occurred only in layer 3 (Fig. 4B). Anterogradely labeled fibers occurred across all layers. Idm1 was architectonically characterized by an outward shift in layers 3 to 5, a more collimated layer 3, and a broader layer 5 populated by smaller and more lanceolate neurons than in Idd5 (Fig. 4B and C). The ventral delimitation of the labeling in Idm1 coincided precisely with the architectonic border with Idm2 in which there was only sparse labeling (Fig. 4B). Idm2 differed from Idm1 by a thinner, sparser and less organized layer 3 and a distinct outward shift of layer 4 (Fig. 5C).

Ventral to Idm2, Idv1 and Idv2 contained dense and moderate anterograde and retrograde labeling, respectively (Fig. 4B). The anterograde labeling occurred in layers 1-3a and the labeled somas were in layer $3 \mathrm{a}$ and $\mathrm{b}$ in Idv1. The anterograde labeling in Idv2 was concentrated in layers $1,3 \mathrm{~b}$ and 5 , and the cell bodies in layer 5, which contrasted with the layer distribution in Idv1. Architectonically, Idv1 had a thinner layer 5-6 and more homogeneous layer 3 (Fig. 5C). Idv2 had even thinner layers 5-6 than in Idv1, and was distinguished from Ivfp by a larger and small-celled layer 3 (Fig. 5C). Ivfp and Ivfa had dense and sparse anterograde labeling and no retrograde labeling. Ivfp had a thin, compact layer 5, thin layer but discernable layer 4, and thin layer 3 made of larger cells than in Idv2. Ivfa overall smaller cells than in Ivfp and no clearly discernable layer 4.

OM42. Anterograde labeling in OM42 occurred in a few dysgranular sub-areas in the middle portion of the insula, and more anteriorly in the rostral portion of Idm3, in Iap1, Ial, Iai, Iapm and Iam (Fig. 3E). The labeling filled the entire extent of Iapl and Iam, and a small fraction of Ial and Iai. Figures 6A-D show the plot of anterogradely labeled terminals overlaid on low magnification photomicrographs of the corresponding BDA-stained sections of the insula, at 4 levels passing through the Idd2 labeling patch $(2500,2000,1500$ and $1000 \mathrm{~mm}$ from the anterior commissure; 0.5-mm interval). Figures 6A'-D' and 7 show the localization of cytoarchitectonic borders on, respectively, low and high magnification photomicrographs of Nissl-stained sections of the insula adjacent to the BDA-stained sections. (Figure 7 focuses on Idd2 and neighboring sub-areas.) The 

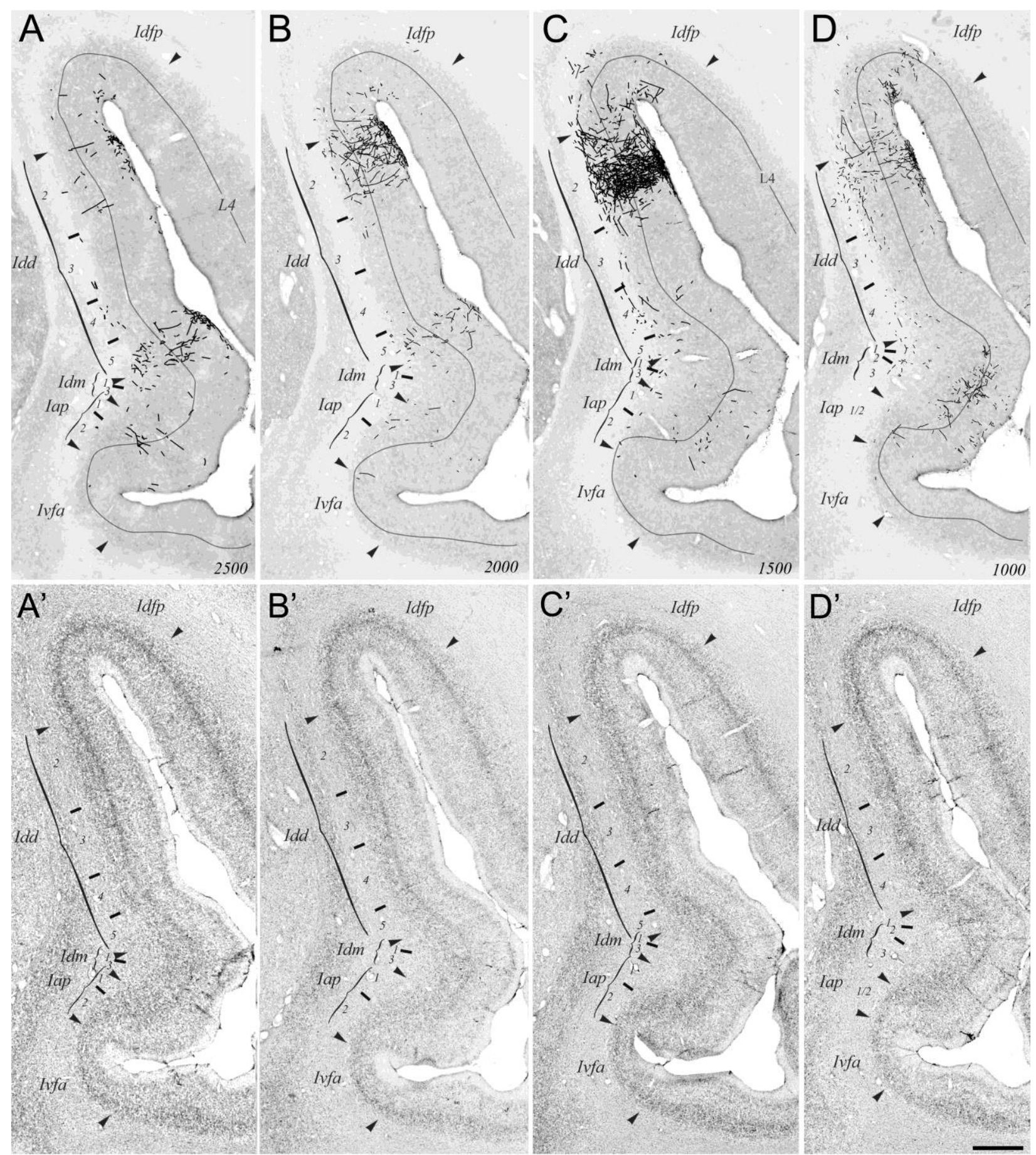

same borders were transposed on the BDA photomicrographs and plots in Figure 6A-D. Most noticeably, a heterogeneous but continuous patch of anterograde labeling formed an anteroposterior strip in Idd2 and, to some extent within Idfp. In addition to the patch in Idd2, anterograde labeling occurred in Idd5 across two consecutive sections (Fig. 6A and B), as well as in Idm1-3 in one section (Fig. 6D). The cytoarchitectonic boundary between Idfp and Idd2 was marked by a thickening layers 3 and 5, and a more diffuse layer 4 in Idd2. The outward shift of 
layer 4 observed in OM85 was not as pronounced in OM42 but still visible for instance in Fig. 7B and C. Conversely, Idd3 had equally thick layers $3 \mathrm{a}$ and $3 \mathrm{~b}$ with sparser, larger and often darker neurons in layer 3b. Layer 5 was thinner and had smaller cells than in Idd2. Idd4 had a more diffuse layer 4 than in Idd3, with a less distinct separation from layers 3 and 5. As in OM85, layer 3a and b were also less separable in Idd4 than in Idd3.

Figure 6. Composite showing the spatial overlap of patches of anterograde labeling with specific cytoarchitectonic subareas in the insular cortex in a case OM42 with an injection of biotin dextran amine (BDA) in the area 111 of the orbital prefrontal cortex (OPFC). A-D. Overlay of the charts of BDA-positive axon terminals on low-magnification photomicrographs of the corresponding BDA-stained sections of the insula across four coronal levels. The localization of the cytoarchitectonic boundaries between areas (arrowheads) and subareas (ticks) is shown for the subareas where anterograde labeling occurred. The distance of each coronal section from the level of the anterior commissure is noted in millimeters, in the bottom right corner of each panel (4500 mm in A to $0 \mathrm{~mm}$ in D). A'-D'. Overlay of the demarcation of the cytoarchitectonic boundaries of the insula on low-magnification photomicrographs of Nissl-stained sections directly adjacent to the BDA-stained sections shown in panels A-D. All areas and subareas are noted. (See abbreviations list.) High-magnification photomicrographs of selected subareas are shown in Figures 9, 10 and 11, allowing clear recognition of the cytoarchitectonic boundaries. (See text.) The asterisks mark the position of blood vessels used as landmarks across adjacent BDA- and Nissl-stained sections. Scale $=1 \mathrm{~mm}$.

OM27. The injection of BDA in area 111 in OM27 produced anterograde labeling in ta dorsalposterior region and in a ventral-anterior region (Fig. 3F). The dorsal posterior labeling included labeling in Idfp, Idd1, 2, 3 and 4. The ventral anterior labeling included Idm2 and 3, Idv1 and 2, Iap1 and 2, and small portions of Iapl, Ial and Iai. Most labeled dysgranular sub-areas contained both anterograde and retrograde labeling. Agranular areas contained mainly anterograde labeling. Figures 8 to 11 focus on sections at four different ap levels $(4500,3000,1500$ and $0 \mathrm{~mm}$ from the anterior commissure; $1.5-\mathrm{mm}$ interval) with labeling in both regions. Figures 8A-D show the plots of BDA-positive terminals overlaid on the low magnification photomicrographs of the corresponding BDA-reacted sections of the insula. Figures 8A'-D' show the cytoarchitectonic boundaries on low magnification photomicrographs of adjacent Nissl-stained sections. Figures 911 show high magnification photomicrographs demonstrating the architectonic features of the subareas containing labeling. 
bioRxiv preprint doi: https://doi.org/10.1101/2020.11.03.361055; this version posted November 4, 2020. The copyright holder for this preprint (which was not certified by peer review) is the author/funder. All rights reserved. No reuse allowed without permission.

Krockenberger et al.: Primate insular stripes

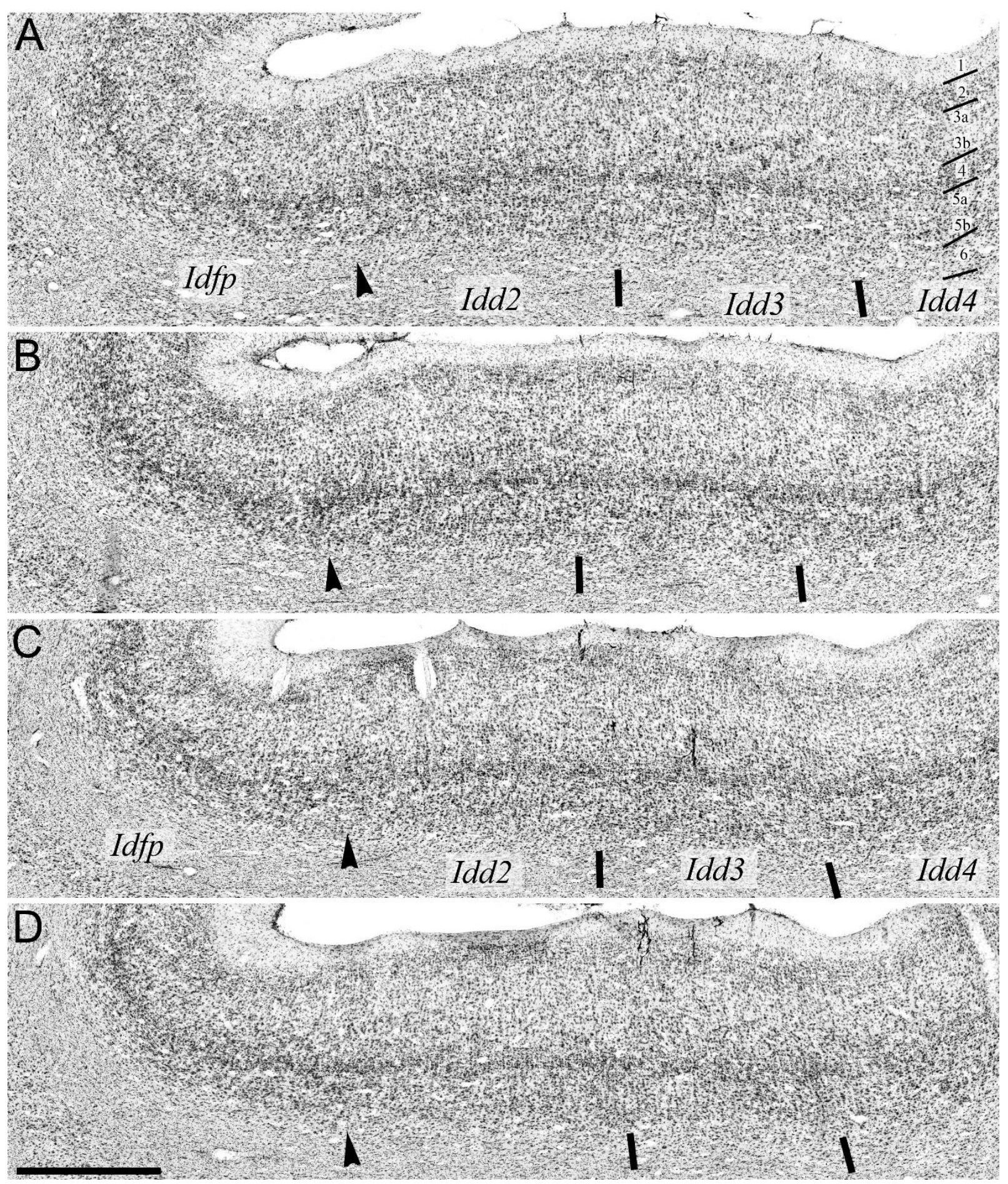

Figure 7. Overlay of the demarcation of cytoarchitectonic boundaries on high-magnification photomicrographs of four consecutive Nissl-stained sections, shown at low-magnification in Figure 6A'-D' in case OM42. The emphasis is on Idfp, Idd2 and Idd3 where anterograde labeling occurred. Areas are separated by an arrowhead. Subareas are separated by tick marks. (See text and abbreviation list.) Scale $=1 \mathrm{~mm}$. 
bioRxiv preprint doi: https://doi.org/10.1101/2020.11.03.361055; this version posted November 4, 2020. The copyright holder for this preprint (which was not certified by peer review) is the author/funder. All rights reserved. No reuse allowed without permission.

Krockenberger et al.: Primate insular stripes
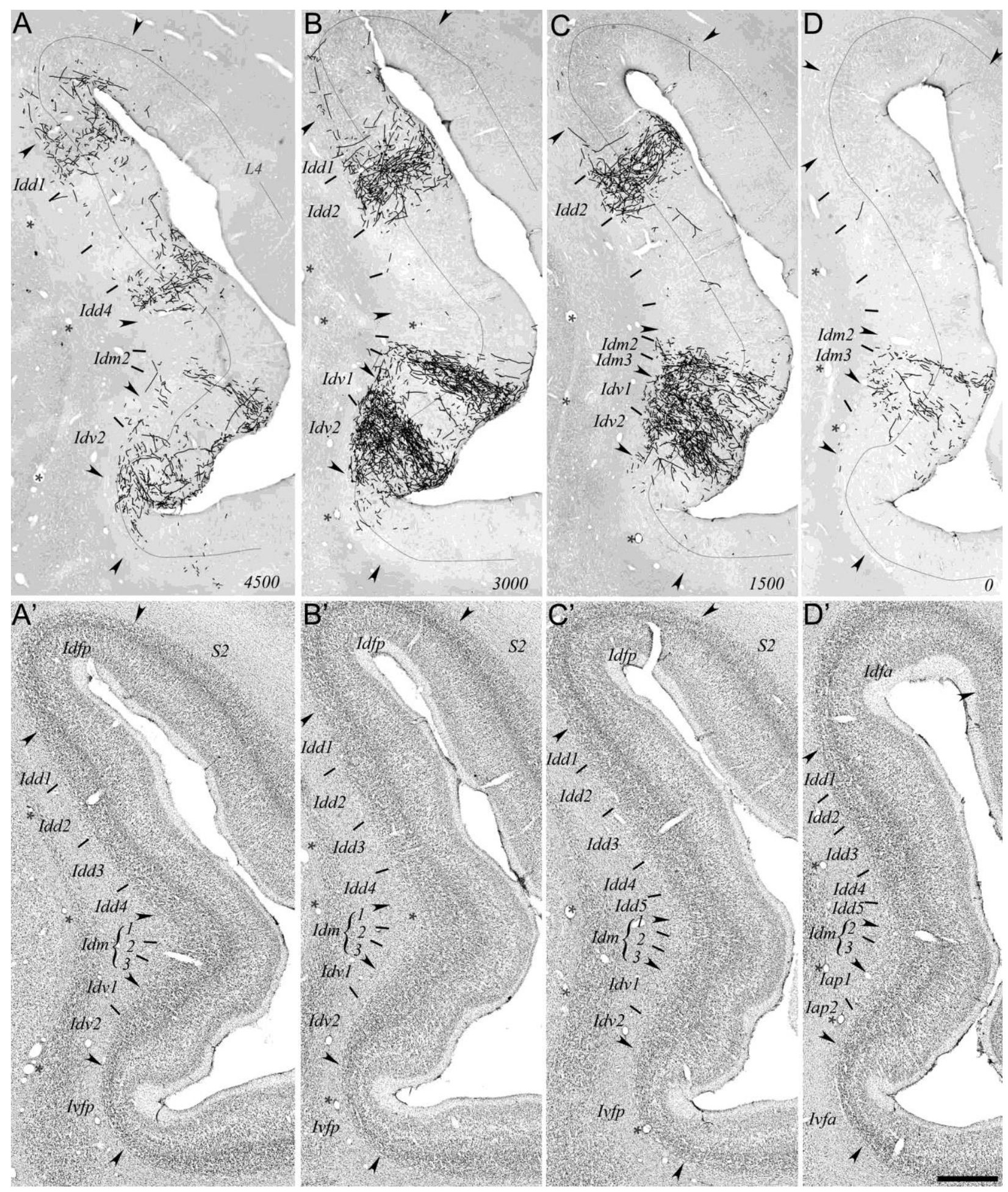

Figure 8. Composite showing the spatial overlap of patches of anterograde labeling with specific cytoarchitectonic subareas in the insular cortex in a macaque monkey (OM27) with an injection of biotin dextran amine (BDA) in the area 111 of the orbital prefrontal cortex (OPFC). A-D. Overlay of the charts of BDA-positive axon terminals on low-magnification photomicrographs of the corresponding BDA-stained sections of the insula across four coronal levels. The localization of the cytoarchitectonic boundaries between areas (arrowheads) and subareas (ticks) is shown for the subareas where anterograde labeling occurred. The distance of 
Krockenberger et al.: Primate insular stripes

each coronal section from the level of the anterior commissure is noted in millimeters, in the bottom right corner of each panel (4500 $\mathrm{mm}$ in A to $0 \mathrm{~mm}$ in D). A'-D'. Overlay of the demarcation of the cytoarchitectonic boundaries of the insula on lowmagnification photomicrographs of Nissl-stained sections directly adjacent to the BDA-stained sections shown in panels A-D. All areas and subareas are noted. (See abbreviations list.) High-magnification photomicrographs of selected subareas are shown in Figures 9, 10 and 11, allowing clear recognition of the cytoarchitectonic boundaries. (See text.) The asterisks mark the position of blood vessels used as landmarks across adjacent BDA- and Nissl-stained sections. Scale = $1 \mathrm{~mm}$.

Idfp contained a small patch of weak to moderate labeling, at the same anteroposterior levels where Idd 1 contained moderate to dense labeling (Fig. 3F and 8A). Anterograde labeling occurred throughout a long segment within the rostrocaudal extent of Idd2, with however a noticeable gap, approximately in the middle of the segment, which coincided with an increase in labeling density in Idd1 (Fig. 3F and 8A-C). This local variation illustrates the complexity or non-triviality of the modular organization of the prefronto-insular connections. Figures 9A-C show high magnification photomicrographs of the dorsal portion of the Nissl-stained sections shown in Figure 8A'-C'. In all three sections, Idd 1 differed from Idd 2 by an overall thinner, denser and less columnar layer 3 with smaller cells than in Idd2, as well as a thinner and less populated layer 5. Idd2 showed sparser but larger cells with a slightly more collimated organization in its broader layer 3 . The border between Idd 2 and Idd 3 was equally abrupt. Layer 3 in Idd3 was thinner with sparser but larger cells and a better distinction between layers $3 \mathrm{a}$ and $3 \mathrm{~b}$. The columnar organization seen in Idd 2 was replaced with a more random organization in Idd3.

Figure 10 shows the cytoarchitectonic borders of Idd4, which contained a patch of anterograde labeling throughout its caudal two thirds (Fig. 8F). In comparison to Idd3, Idd4 had a thinner and more homogeneous layer 3 made of darkly stained pyramidal somas and a less distinct layer 4. The border between Idd4 and Idm1 was rather sharp, with a denser layer 3 made of smaller and more collimated cells in Idm1, an outward shift in layer and a markedly thicker layer 5 populated with smaller neurons, compared to Idd4.

Figure 9. [next page] Overlay of the demarcation of cytoarchitectonic boundaries on high-magnification photomicrographs of three Nissl-stained sections, shown at low-magnification in Figure 8A'-C' in case OM27. The emphasis is on subareas Idd1 and Idd2. Areas Idfp and Idd are separated by an arrowhead. Subareas 1, 2, 3 and 4 of Idd are separated by tick marks. (See text and abbreviation list.) Scale $=1 \mathrm{~mm}$. 

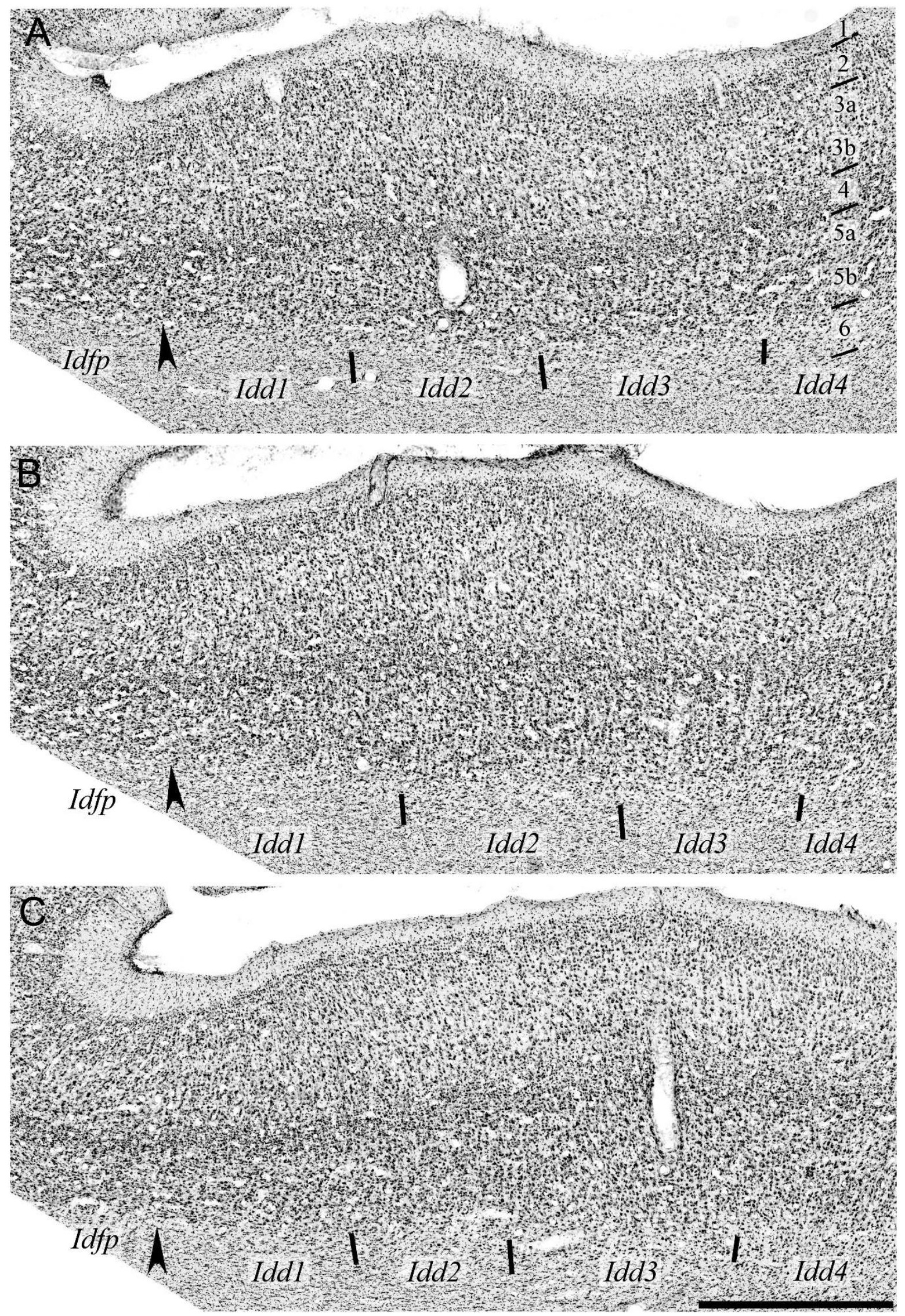


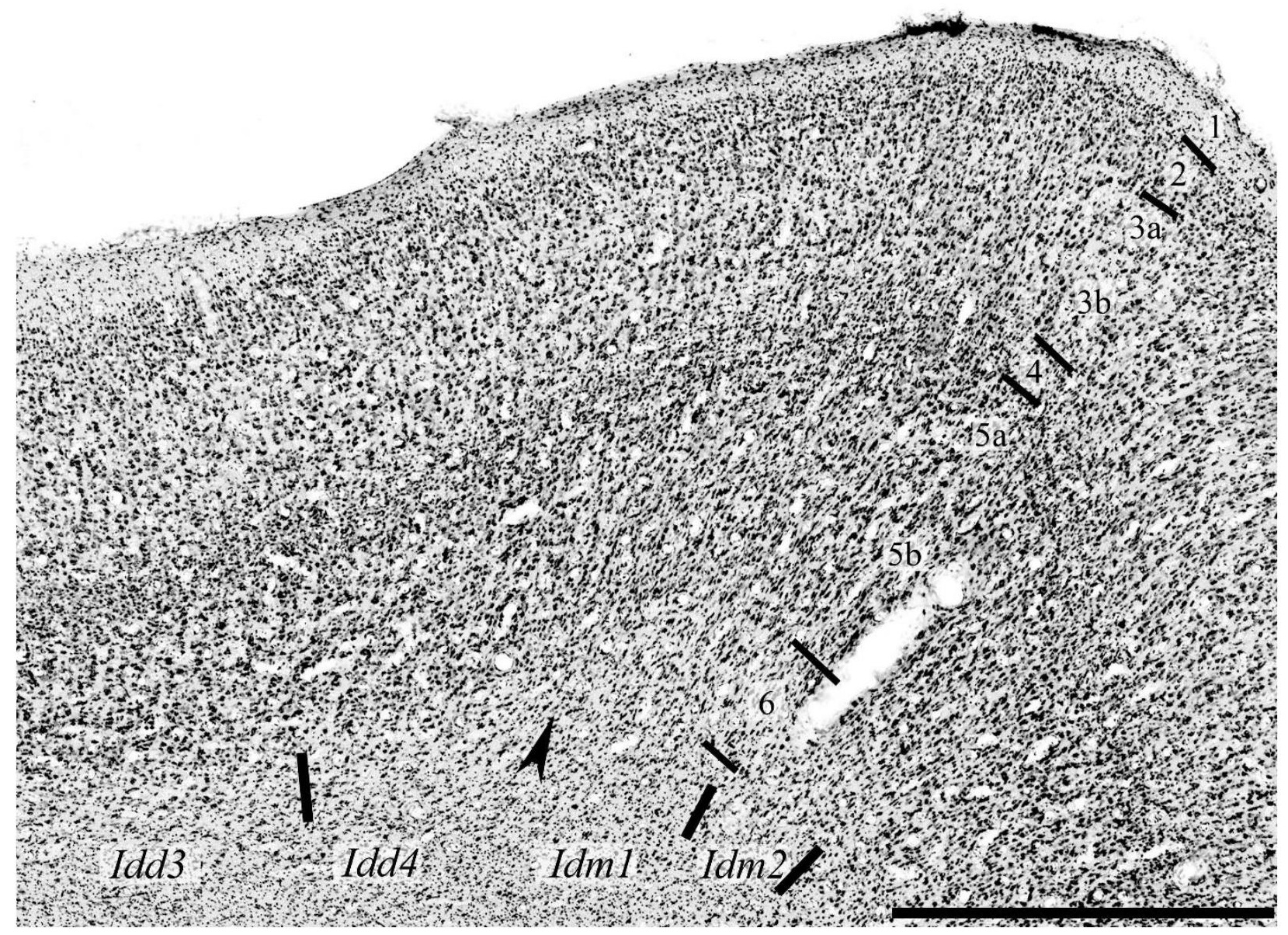

Figure 10. Overlay of the demarcation of cytoarchitectonic boundaries on high-magnification photomicrographs of one Nisslstained section, shown at low-magnification in Figure 8A' in case OM27. The emphasis is in on subareas Idd4. Subareas 3 and 4 of Idd are separated by tick marks. Areas Idd and Idm are separated by an arrowhead. (See text and abbreviation list.) Scale $=1$ $\mathrm{mm}$.

In the mound area, Idm1 was completely devoid of labeling, conversely to $\operatorname{Idm} 2$, which contained dense labeling, with an abrupt dorsal delimitation that perfectly coincided with the cytoarchitectonic boundary between Idm1 and Idm2 (Fig. 8A-C). The boundary was marked by a subtle but definite fault line in the layer organization. The small and lanceolate cells in layers 2 and 3 in Idm1 were replaced with sparser, larger and rounder cells in Idm2. The discrete columnar organization was replaced with a random organization, in both layers 3 and 5. Idm3 had a varying density of anterograde labeling, from weak to moderate. However, the labeling was always distinct from the labeling in Idm2 and Idv1. The boundary with Idm2 was marked by a thinner layer 3 with even sparser neurons and a thinner homogeneous layer 5. Idv, as a whole, contained moderate to dense labeling with no clear distinction between Idv1 and Idv2, except perhaps for a progressive 
bioRxiv preprint doi: https://doi.org/10.1101/2020.11.03.361055; this version posted November 4, 2020. The copyright holder for this preprint

(which was not certified by peer review) is the author/funder. All rights reserved. No reuse allowed without permission.

increase in density from Idv2, posteriorly, to Idv1, more anteriorly (Fig. 2F). The boundary between Idm3 and Idm1 was marked by a thickening of layer 3, with smaller and lanceolate cells, and less discernible layers 2 and 4 . The boundary between Idv1 and Idv2 was marked by a sharp reduction in the thickness of layers 2, 3 and 5 (Fig. 11A-C). Finally, Ivfp had only sparse labeling at the border with Idv2 in a few sections. The architectonic border was marked by further thinning of all layers, and a sparser layer 3.

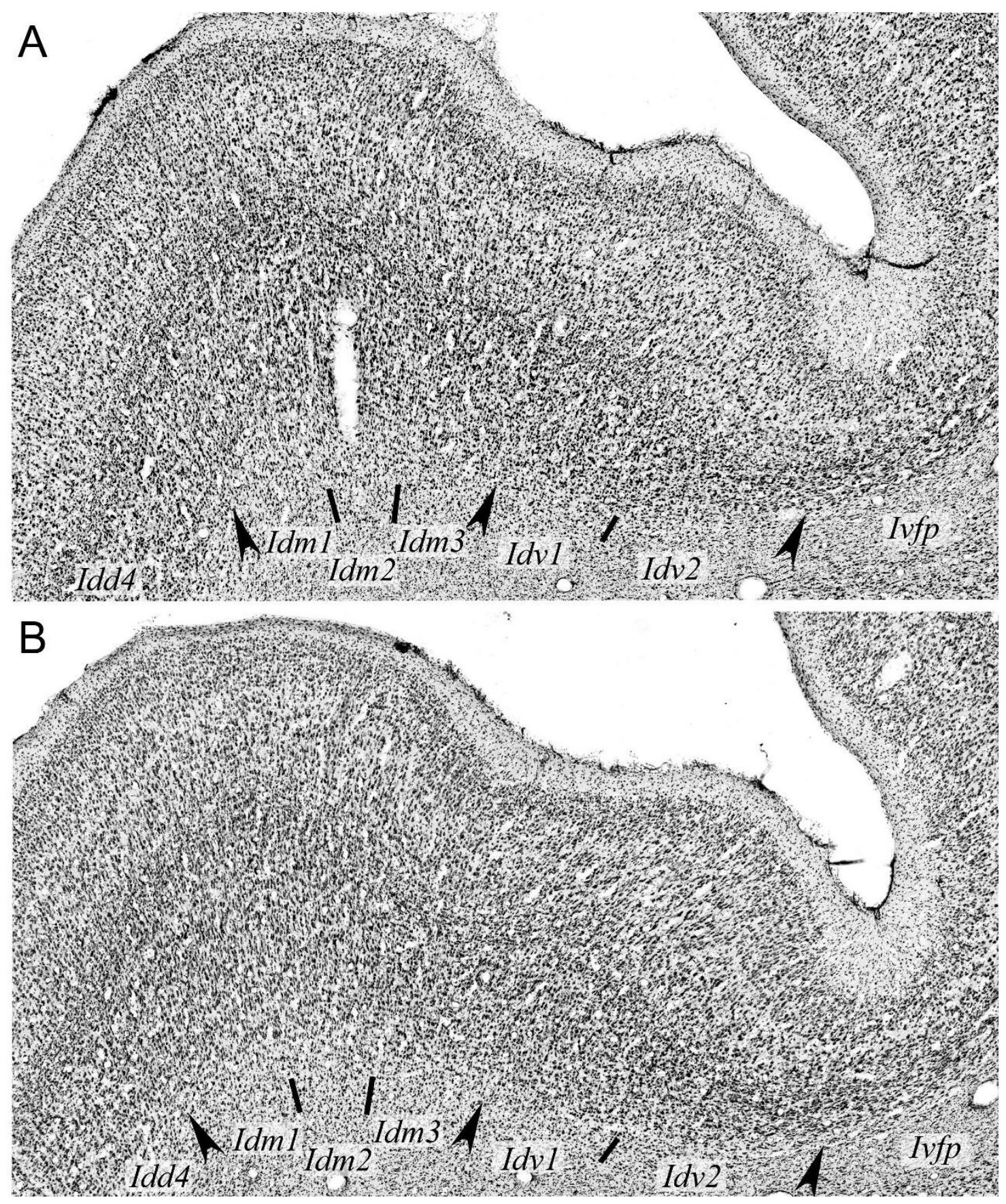


Figure 11. Overlay of the demarcation of cytoarchitectonic boundaries on high-magnification photomicrographs of four Nisslstained sections, shown at low-magnification in Figure 8A'-D' in case OM27. The emphasis is in on the subareas of areas Idm and Idv. Subareas are separated by tick marks. Areas are separated by arrowheads. (See text and abbreviation list.) Scale $=1 \mathrm{~mm}$.

OM30. The anterograde labeling produced by the injection of BDA in area 121 was limited to a few patches (Fig. 3G). Figure 12 illustrates the sharp delimitation of one of these patches and its overlap with cyto- and myelo-architectonic boundaries of Idd3. Idd3 was characterized by a larger layer 3 than in Idfp, with distinct and equally thick layers 3a and b. Idd4 had a more homogenous layer 3 and a broader and also rather homogenous layer 5 (Fig. 12B and D). (Idd5 had a markedly sparser layer 3, compared to Idd4. Idm1 had broader layers 3 and 5 made of small, lanceolate cells arranged in narrow columns.) In the Gallyas stain, Idd3 had longer and coarser radial/vertical fibers than in Idfp and a diffuse outer band of Baillarger. Idd4 had even longer and coarser radial fibers and its outer band of Baillarger was sifted outwardly. (As in prior cases, the myelin stain density increased in Idd5 and the outer band of Baillarger was shifted inward and less distinct than in Idd4. Idm1 had very long, distinct and thin radial fibers.)

OM69. The injection of LY in OM69 produced anterograde and retrograde labeling in Idfp and Idfa, over a large region in the dorsal and to a lesser extent mound dysgranular areas, in Ivfa, Iapl, Iai and Iapm (Fig. 3H). Figure 13 illustrates the overlap of several adjacent patches of anterograde (Fig. 13A) and retrograde (Fig. 13B) labeling with cyto- and myelo-architectonic dorsal and mound dysgranular subareas. Indeed, a close comparison with adjacent Nissl- and Gallyas-stained sections revealed that the labeling was distributed across four distinct adjacent dysgranular subareas (Idd3, Idd4, Idd5 and Idm1) with a distinct pattern of anterograde and retrograde labeling in each subarea. From dorsal to ventral, Idd2 contained only sparse anterogradely labeled terminals, with a shape delimitation with Idd3 which contained conspicuous anterograde and retrograde labeling (Fig. 13A and B). The anterograde labeling in Idd3 was located mainly in layer $6,5 \mathrm{~b}$ and $3 \mathrm{~b}$, with sparser labeling in the other layers. The retrograde labeling was of moderate intensity (see Fig. 2 for reference) and located in both layer $5 b$ and 3. Idd 4 also contained anterograde and retrograde labeling. However, the anterograde labeling in Idd4 was slightly sparser than in Idd 3 and the labeled somas occurred exclusively in layers 5a and 6. The anterograde labeling in Idd5 was similar to Idd4, but the retrograde labeling was located in layers 3, 5 and 6, and it was of stronger intensity than in Idd3. Finally, the labeling in Idm1 was characterized by a thin and almost continuous band of labeled somas from layer 6 to $3 \mathrm{~b}$, and a bundle of terminals 
bioRxiv preprint doi: https://doi.org/10.1101/2020.11.03.361055; this version posted November 4, 2020. The copyright holder for this preprint

(which was not certified by peer review) is the author/funder. All rights reserved. No reuse allowed without permission.

26

Krockenberger et al.: Primate insular stripes

spanning across all layers with the exception of layer 3a. Conversely, Idm2 contained only very sparse anterograde labeling and no retrograde labeling.

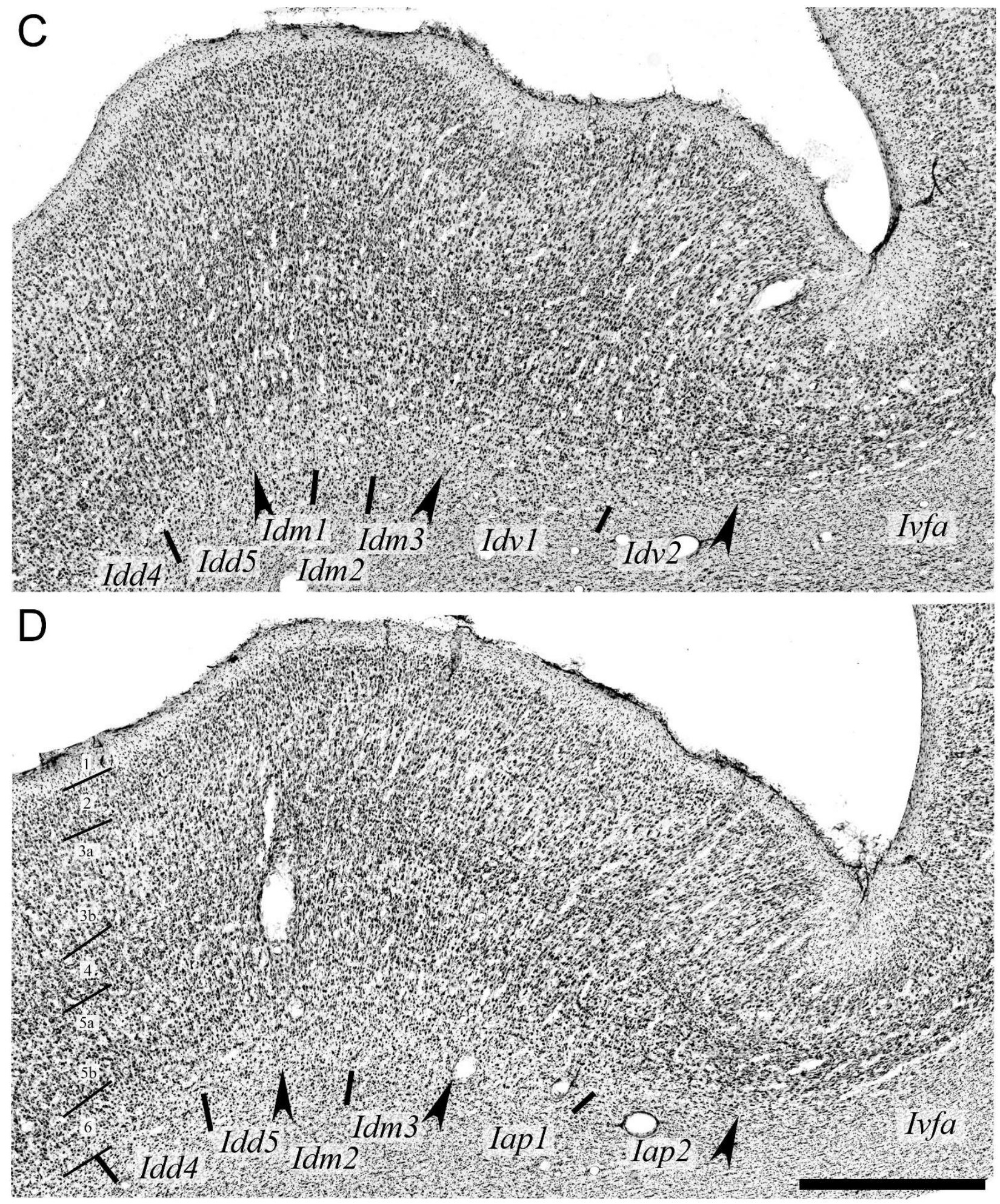

Figure 11 (continued) 
bioRxiv preprint doi: https://doi.org/10.1101/2020.11.03.361055; this version posted November 4, 2020. The copyright holder for this preprint

(which was not certified by peer review) is the author/funder. All rights reserved. No reuse allowed without permission.

Krockenberger et al.: Primate insular stripes

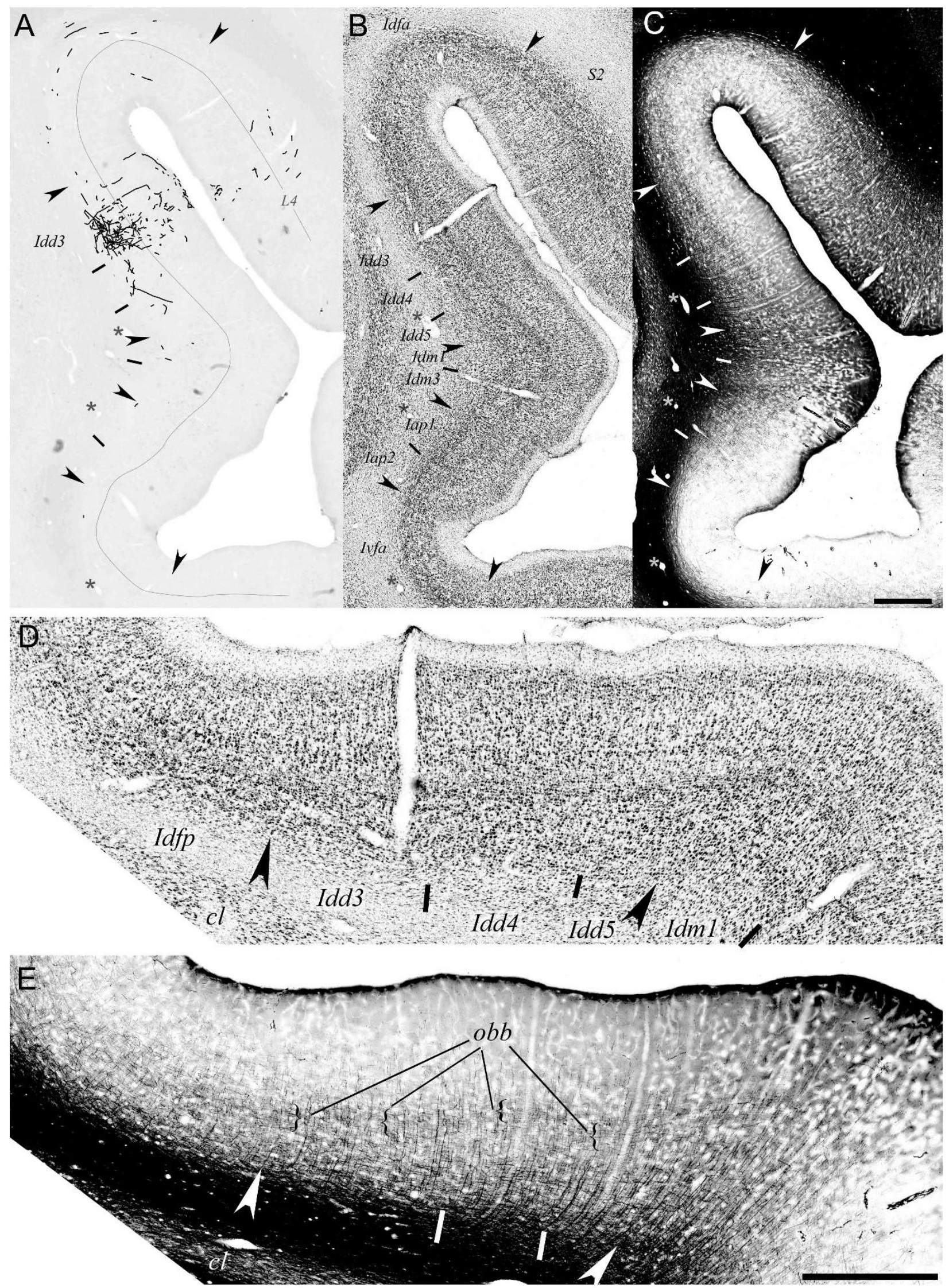


Krockenberger et al.: Primate insular stripes

Figure 12. Composite showing the spatial overlap of patches of anterograde labeling with specific cyto-and myelo-architectonic subareas in the insular cortex in a macaque monkey (OM30) with an injection of biotin dextran amine (BDA) in the area $12 \mathrm{~m}$ of the orbital prefrontal cortex (OPFC). A. Overlay of the charts of BDA-positive axon terminals on low-magnification photomicrographs of the corresponding BDA-stained sections of the insula. $\boldsymbol{B}$ and $\boldsymbol{C}$. Overlay of the demarcation of, respectively, the cyto- and myelo-architectonic boundaries of the insula on low-magnification photomicrographs of Nissl-and Gallyas-stained sections directly adjacent to the BDA-stained section shown in panel A. All areas and subareas are noted. (See abbreviations list.) $\boldsymbol{D}$ and $\boldsymbol{E}$. High-magnification view of the cyto- and myelo-architecture and boundaries in the dorsal half of the insula from panels $B$ and $C$. In panel $E$, the outer band of Baillarger is indicated $(o b b)$. Scale $=1 \mathrm{~mm}$.

The cytoarchitecture in OM69 was not as obvious as in the other cases due to noise created by a contamination of the tissue with stained blood cells that did not wash optimally during the perfusion. Nevertheless, the main modular cytoarchitectonic features could be distinguished (Fig. 12C and E) and matched with the myeloarchitectonic features (Fig. 13D and F). The borders between the distinct dorsal dysgranular subareas were marked by changes in the thickness and demarcation of granular layer 4, as well as some variations in the cellular organization of layer 3 and to some extent 5. Like in the other cases, layer 4 was thin in Idd2, thicker and more distinct in Idd3, very diffuse in Idd4, and broader and slightly more recognizable in Idd5. The border between Idd 2 and Idd 3 was also marked with a sudden decrease is cell density in layer 3 as well as a thinner but large-celled layer 5 in Idd3, compared to Idd2. In addition of the diffuse layer 4, Idd4 had a thinner and more homogeneous layer 3 than in Idd3. The border between Idd4 and Idd5 was subtly marked by an outward shift in layer 5, a thinner layer 3 and broader layer 5 with smaller cells than in Idd4. Idm1 had overall smaller, more lanceolate and collimated cells, with a broader layer 5, similar to other cases. In parallel with the changes in the cellular appearance of layer 4, the outer band of Baillarger was very distinct in Idd2, weak in Idd3 and Idd4, and not distinct in Idd5 (Fig. 13F). Idd 2 had a coarse mesh of stained fibers in layers 5 and 6 which differed from the coarse and more radial (vertical) fibers in Idd3. The difference between Idd 3 and Idd4 was tenuous with only slightly less horizontal fibers crossing the radial fibers in Idd4. Idd5 had longer and straighter radial fibers than in Idd4. Idm1 was very distinct, with long radial fibers crossing the weak band of Baillarger into layer 3. 
bioRxiv preprint doi: https://doi.org/10.1101/2020.11.03.361055; this version posted November 4, 2020. The copyright holder for this preprint (which was not certified by peer review) is the author/funder. All rights reserved. No reuse allowed without permission.

Krockenberger et al.: Primate insular stripes
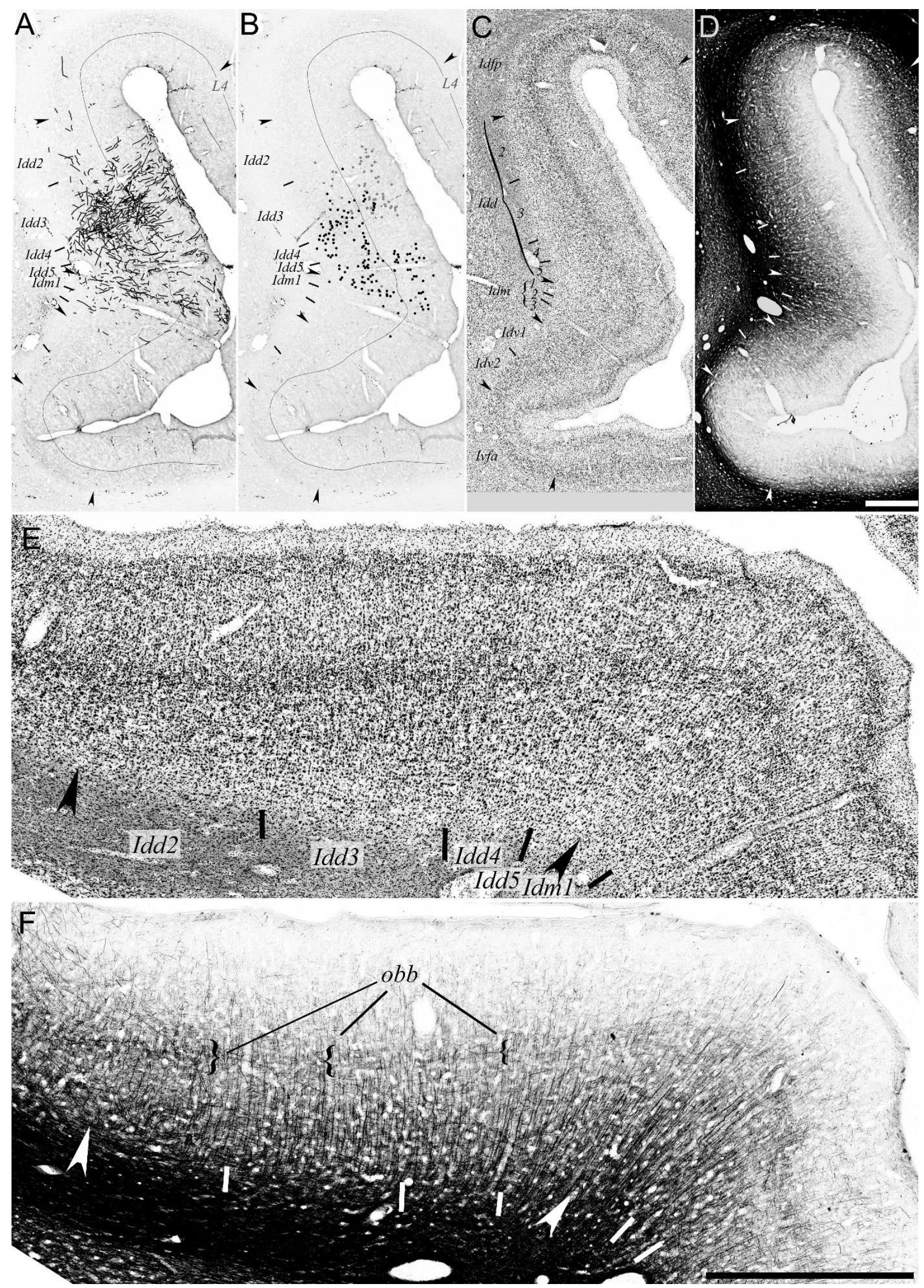
Krockenberger et al.: Primate insular stripes

Figure 13. Composite showing the spatial overlap of patches of anterograde labeling with specific cyto- and myelo-architectonic subareas in the insular cortex in a macaque monkey (OM69) with an injection of lucifer yellow (LY) in the area 12r of the orbital prefrontal cortex (OPFC). A-B. Overlay of the charts of LY-positive axon terminals (A) and cell bodies (B) on low-magnification photomicrographs of the corresponding LY-stained sections of the insula. C-D. Overlay of the demarcation of the cyto- (C) and myelo- (D) architectonic boundaries of the insula on low-magnification photomicrographs of Nissl- and Gallyas-stained sections directly adjacent to the BDA-stained section shown in panel A. E-F. High-magnification view of the cyto- and myelo-architecture and boundaries in the middle of the insula from panels $\mathrm{C}$ and D. In panel F, the outer band of Baillarger is indicated (obb). (See abbreviations list.) Scale $=1 \mathrm{~mm}$.

\section{Discussion}

The present neuroanatomical report demonstrates the close spatial relationship between the localization of architectonic boundaries and the delimitation of discrete regions of anterograde and/or retrograde labeling in the insular cortex in the macaque monkey. The architecto-hodological overlap validates the recent architectonic parcellation of the classical granular, dysgranular and agranular sectors of the macaque insula into smaller and rather sharply delimited areas and subareas (Evrard et al. 2014), which is consistent with modern reports demonstrating a similar overlap in other cortical lobes (e.g., Lewis and Van Essen 2000; Kaas 2002; Price 2007; Qi et al. 2008). Conversely, the overlap definitely refutes a former conclusion that "connectivity patterns" have "negligible" value for the determination of cytoarchitectonic borders in the primate insula (Gallay et al. 2012).

Although quite straightforward in appearance, the present finding represents a major progress and novelty in the examination of the primate insula. It will become of paramount importance for models of insular processing. Even if the 'overlap' may not be the rule for all projections to and from the insula (see below), it provides for the first time a clear structural support for the notion that the primate insulo-insular processing occurs within a refined modular Bauplan, in which each dysgranular subarea likely integrates interoception with functionally distinct polymodal and limbic afferent activities (Evrard and Craig 2015; Evrard 2018). 
Krockenberger et al.: Primate insular stripes
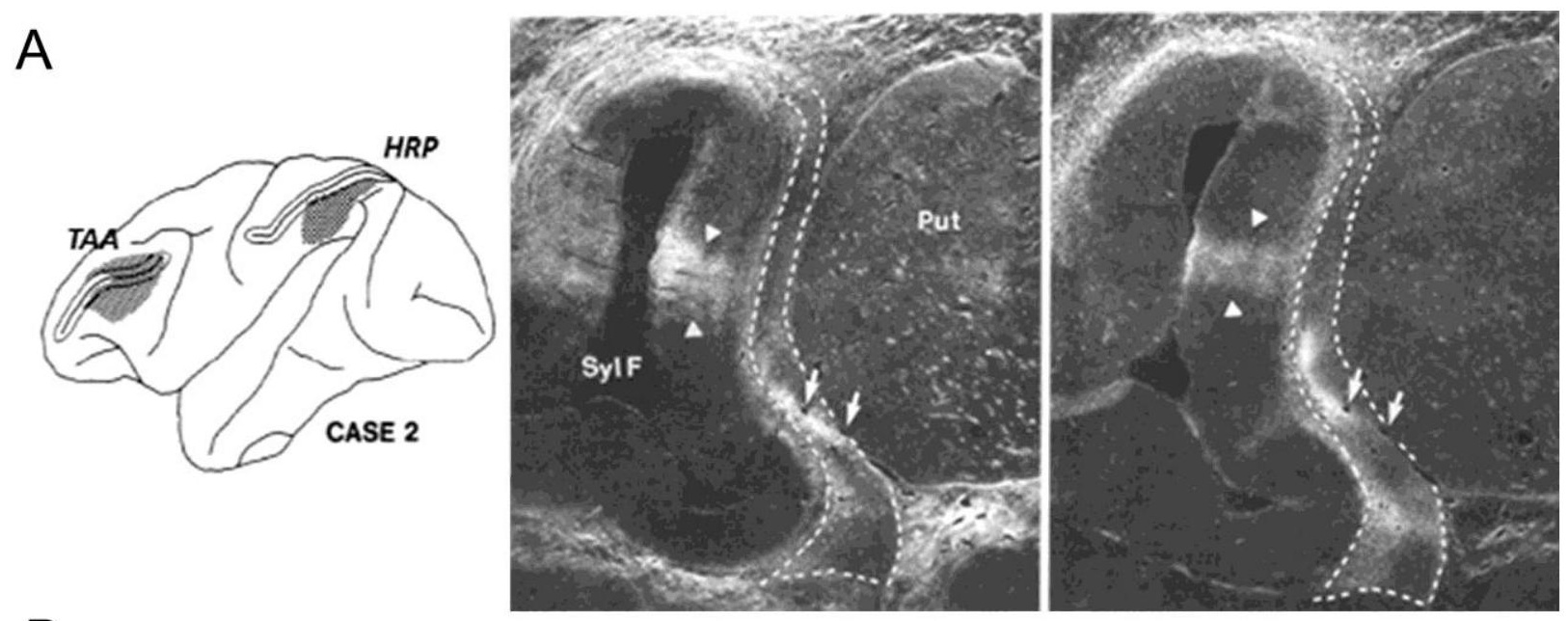

B

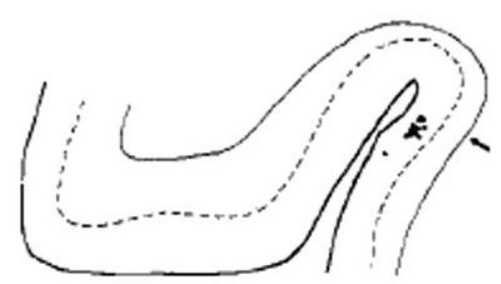

6800

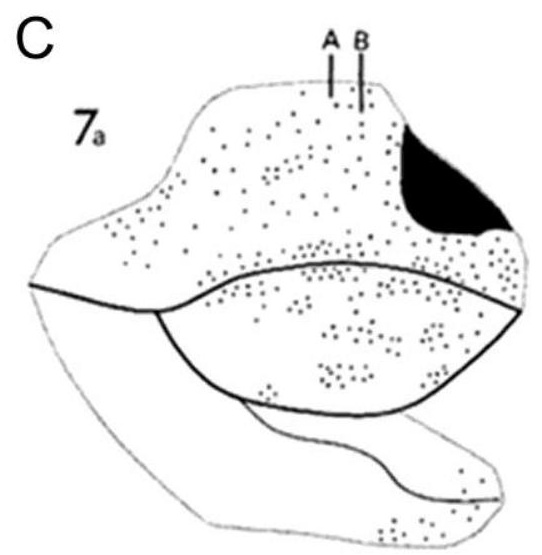

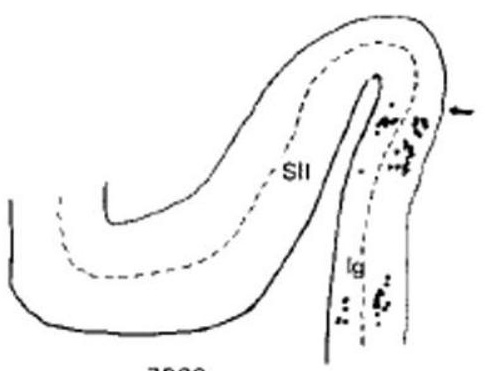

7200

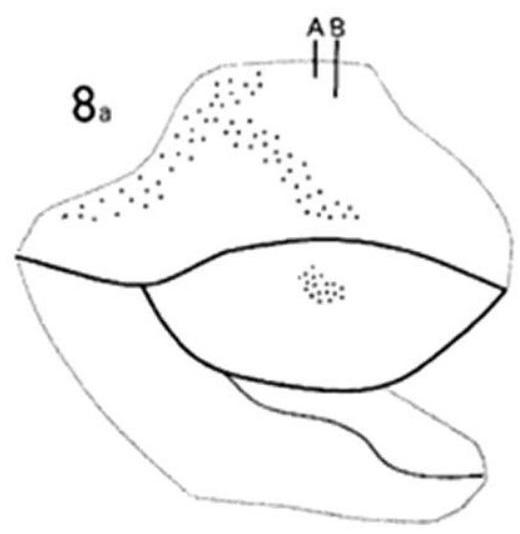

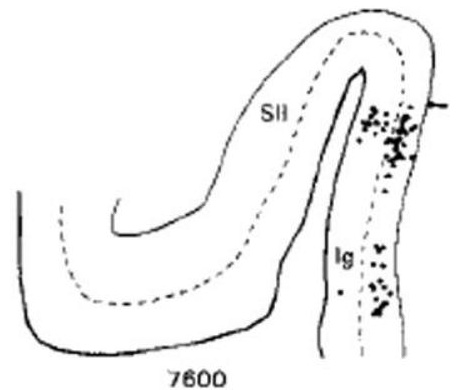

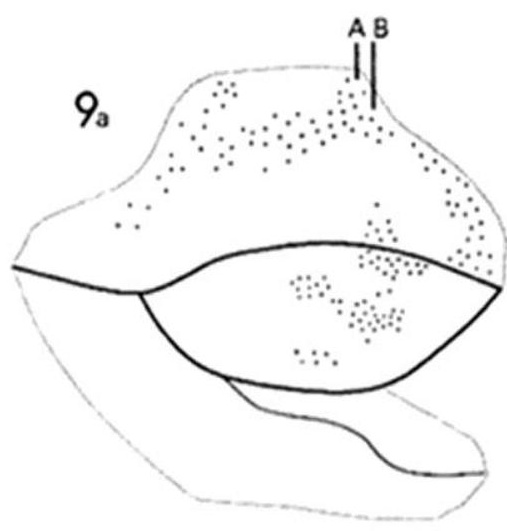

Figure 14. A. Coronal photomicrographs showing patches of tritiated amino acid (TAA, left) and horseradish peroxidase (HRP, right) anterograde labeling in the insula with injections in the prefrontal and posterior parietal cortices (Adapted with permission from Selemon \& Goldman-Rakic, 1988). B. Distribution of DY-labeled cells on selected coronal sections through the dorsal dysgranular insula after injections in the distal toe region of area $3 \mathrm{~b}$. Each dot represents the location of one retrogradely labeled cell (Adapted with permission from Burton et al., 1995). C. Flat map representation of "stripes" of retrograde labeling in the insula in 3 monkeys with injection of HRP in distinct parts of the brain. The injections were made in the frontoparietal operculum (7a), posterior parietal cortex (8a) and lateral prefrontal cortex (9a) (Adapted with permission from Mesulam and Mufson, 1982). 


\section{Comparison with prior tract-tracing studies in macaques}

Numerous prior tract-tracing studies reported labeling in the insula (e.g., Burton and Jones 1976; Jones and Burton 1976; Pandya et al. 1981; Mesulam and Mufson 1982; Mufson and Mesulam 1982; Cavada et al. 1984; Matelli et al. 1986; Selemon and Goldman-Rakic 1988; Preuss and Goldman-Rakic 1989; Deacon 1992; Carmichael and Price 1995; Chikama et al. 1997; Cipolloni and Pandya 1999; Stefanacci and Amaral 2000; Lavenex et al. 2002; Fudge et al. 2005; Borra et al. 2008). The labeling never entirely 'filled' one of the three classical cytoarchitectonic sectors. It was instead heterogeneous and a close examination of figures from these studies readily reveals a more complex organization that often included 'patches' and 'stripes' fitting with the present finding.

One of the most edifying examples comes from a study by Selemon and Goldman-Rakic (1988). In this study, large injections of horseradish peroxidase in prefrontal cortex and tritiated amino acid (TAA) in posterior parietal cortex produced sharply delimited and overlapping patches of anterograde labeling in an insular region that could correspond to one of the architectonic subareas of our dorsal dysgranular area (Fig. 14A). In another example, Burton and colleagues (1995) showed "stripes" of retrograde labeling that were continuous across almost several mm. They located these stripes in the granular insula ("Ig" in Fig. 14B) but they could very well correspond to the first and third (Idd1 and 3) or second and fourth (Idd2 and 4) subareas of our dorsal dysgranular area. This example directly supports the continuous anteroposterior patches of labeling reported in the present study. In a final example, large injections of TAA in the frontal operculum, posterior parietal cortex or prefrontal cortex also produced rather heterogeneous anterograde labeling in the insula (Mufson and Mesulam 1982). The flat map illustrations of this labeling (Fig. 14C) clearly denoted small anteroposterior stripes that were similar to the stripes reported in the present contribution (Fig. 3).

In their seminal tract-tracing studies of the cortico-cortical connections of the macaque insula, Mesulam and Mufson (1982b) divided the insula into two vast hodological territories (Fig. 1F). They proposed an anteroventral 'limbic' territory including the agranular insula and the rostral half of the dysgranular insula separated by a gradual transition from a posterodorsal 'auditorysomesthetic-skeletomotor' territory including roughly the caudal half of the dysgranular insula and the granular insula. While this general anteroposterior progression will likely remain valid and valuable (Cerliani et al. 2010; Cerliani et al. 2012), the present finding recommends a thorough 
reexamination of all existing and viable tract-tracing material with labeling in the insula. The examples above, and in particular the case of dual anterograde labeling in one single discrete region with two very distinct injections (Fig. 14A), readily announce that such reexamination would definitely enlighten our understanding of both the anatomical and functional organization of the primate insula.

\section{Comparison with other species}

We already reviewed in our prior contribution the major organizational and topographical differences between the monkey, cat and rat insulae, including for instance the absence of a distinct interoceptive spinothalamocortical pathway (see Discussion in Evrard et al. 2014). Tract-tracing studies of the rat insula demonstrated optimal overlaps between architectonics and hodology (e.g., Allen et al. 1991; Shi and Cassell 1998; Jasmin et al. 2004). However, this overlap was limited to the three-five classical architectonic areas of the rat insula and did not reveal thinner 'modular' parcellation. In addition, the projections from the putative analog of the monkey prefrontal cortex targeted mainly the agranular area of the insula (Allen et al. 1991; Gabbott et al. 2003), whereas in macaque the prefrontal cortex projects in a very heterogeneous manner chiefly to the dysgranular areas and to a lesser extent to the agranular areas. Although an overall involvement in homeostasis can still offer points of comparison across species and lead to the enunciation of general analog principles, the modular and hodological organization of the macaque insula is, by far, more elaborate than that of the rodent, and justifies using caution when comparing this structure between primate and non-primate species. The addition of areas and subareas in the macaque insula likely reflects major evolutionary adaptations and additions of new functions, as already proposed for other systems (Kaas 2002; Kaas 2004).

The existence of multiple sharply-delimited insular areas is common to our work and these of Rose (1928), Brockhaus (1942), and Kurth et al. (2010) in humans (Fig. 1) (See Discussion in Evrard et al. 2014). Additional architectonic examinations of the human insula are now needed to analyze (1) whether the three dysgranular areas posterior to the central sulcus (Kurth, Eickhoff, et al. 2010) contain finer subdivisions, (2) whether these subdivisions continue passed the central sulcus into the small gyri (which have not yet been mapped) or are limited to the posterior long gyri, (3) whether these putative posterior subdivisions are aligned parallel to the dorsal fundus or to the axis of the long gyri, (4) whether the anterior small gyri and the 'frontoinsula' are architectonically divided into more areas than in macaques as suggested by their recent 
disproportional expansion of the anterior insula in humans (Bauernfeind et al. 2013), and, finally, (5) whether the von Economo neurons which seem to occur in only one architectonic area in macaques (Horn et al. 2017) do indeed occur in more than one area in humans, as suggested by our preliminary study (Horn and Evrard 2018; Horn and Evrard, in preparation). Recent functional studies in humans suggested the presence of several distinct anatomical and functional domains within the human insula (Kurth, Zilles, et al. 2010; Deen et al. 2011; Kelly et al. 2012). Continuous efforts to obtain more accurate functional maps of the insula using high-resolution fMRI and to compare these with maps obtained in the macaque monkey may help decipher fixed homologies from species-specific innovations.

\section{Cortical patterns and insular map}

The cerebral cortex contains a series of maps that reflect the topography and superposition or separation of modalities within distinct sensory systems. These maps tend to represent peripheral changes and discontinuities in 'primary' sensory cortices and evolve towards more 'abstract' polymodal maps when progressing towards higher association areas. The organization of these maps, regardless of the level of integration, varies greatly, depending on the sensory modality, underlying connection patterns, species, etc. (Swindale 1990; Horton and Adams 2005; Kaas 2012). In the present study, the fine dysgranular sub-areas in the insular map do not refer to highly regular and periodic columnar patterns such as these observed in the visual or somatosensory cortex in some species (Mountcastle et al. 1957; Hendrickson 1985; Purves et al. 1992; Mountcastle 1997). Although we previously defined our dysgranular subareas as "modules" (Evrard et al., 2014), our subareas are unlikely to represent receptive field discontinuity such as these observed in the "modules" put forward by Kaas in sensory areas across modalities and species (Kaas 2012). The insular "patches" and "stripes" in the present study bear resemblance with prior patterns of periodic intrinsic cortico-cortical projections (Levitt et al. 1993; Lund et al. 1993) and interdigitated extrinsic projections to the frontal association cortex (Goldman-Rakic and Schwartz 1982). However, these patterns occurred within single areas without evidence of overlap with finer sub-areal architectonic parcellations. While repeatability and complementary interdigitating are not excluded, one of the large prefrontal injections labeled only one small region within a subarea without any evidence of repetition (OM30), while one of the small injections labeled multiple adjacent patches without leaving much room for an interdigitated pattern (OM27). 
Rather, as detailed in our earlier model, we suggest that the fine horizontal 'stripe-like' partition of the insula proper reflects the occurrence of a serial processing stream where interoception is integrated with multi-modal activities in order to provide a rather unified representation of interoceptive, exteroceptive and teloreceptive activities of the organism (Evrard and Craig 2015; Evrard 2018; Evrard 2019).

The dorsal fundus of the insular cortex is the terminus of a primate-specific spinothalamocortical pathway encoding the physiological state of the body and constitutes the "primary interoceptive cortex" (I1) (Craig 2002). In our working model of the insula, each horizontal dysgranular subarea receives inputs from I1 and integrates them with afferent activities from selective sets of cortical and subcortical regions (Evrard and Craig 2015; Evrard 2018). This dorsoventral homotopic insulo-insular communication is supported by recent tract-tracing in our lab, showing that one small injection of tracer in a restricted portion of I1 produces labeling in equally restricted portions of the dysgranular subareas at the same anteroposterior levels (Welzel and Evrard, unpublished observations; see also ). This trans-areal process is consistent with the idea that the representation of core affect and sentient feelings in the anterior insula derives from the integration of ongoing environmental signals with interoceptive and homeostatic activities in the posterior and middle insula (Evrard and Craig 2015).

\section{Acknowledgments}

This work was supported by the Werner Reichardt Centre for Integrative Neuroscience (CIN) at the Eberhard Karls University of Tübingen (CIN is an Excellence Cluster funded by the Deutsche Forschungsgemeinschaft [DFG] within the framework of the Excellence Initiative EXC 307) and by the Max Planck Society. Thanks to Prof. Dr. Joseph L. Price for allowing us to use his valuable tract-tracing collection and Prof. Dr. Arthur Lowey for providing generous access to his lab and microscopes, both at the Washington University of Saint-Louis, MO, USA. 


\section{Abbreviations}

BDA Biotinylated dextran amine

CTb Cholera toxin subunit B

FR Fluororuby

Iai intermediate agranular area of the insula

Ial lateral agranular area of the insula

Iam medial agranular area of the insula

Iap1-2 posterior agranular area of the insula, subareas 1 and 2

Iapl posterior lateral agranular area of the insula

Iapm posterior medial agranular area of the insula

Idd1-5 dorsal dysgranular area of the insula, subareas 1 to 5

Idfa anterior (granular) area of the dorsal fundus of the insula

Idfp posterior (granular) area of the dorsal fundus of the insula

Idm1-3 mound dysgranular area of the insula, subareas 1 to 3

Idv1-2 ventral dysgranular area of the insula, subareas 1 and 2

ILS Inferior limiting sulcus of the insula

Ivfa anterior (agranular) area of the ventral fundus of the insula

Ivfp posterior (dysgranular) area of the ventral fundus of the insula

LY Lucifer yellow

SLS superior limiting sulcus of the insula 


\section{Figure legends}

Figure 1. Flat maps drawings of the insular cortex showing the delineation of distinct sectors, areas or sub-areas in seven different cyto- and/or myelo-architectonic studies in humans or cercopithecine monkeys. A. Cytoarchitectonic division of the human insula into one anterior agranular (I. ant.; Ia) and one posterior granular (I. post.; Ig) sector (adapted with permission from Brodmann 1909). B. Myeloarchitectonic division of the human insula in which six myeloarchitectonic fields (i1-i6) were also cytoarchitectonically isocortical (or granular; $I g$ ) and seven others (ai1-i7) allocortical (or agranular; Ia) (adapted with permission from Vogt 1911). C. Cytoarchitectonic division of the hamadryas baboon (Papio hamadryas) insula into two agranular (ai7 and ai8; Ia), two dysgranular (ai5 and ai6; Id), and fourteen granular areas (i1-14; Ig) (adapted from Rose 1928). Rose's agranular and dysgranular sectors contain two (ai1 and ai10) and seven (ai2-6, ai9) additional agranular and dysgranular areas, respectively, that are not shown in this figure. D. Cyto- and myelo-architectonic division of the human insula into two allocortical (agranular; $I a$ ), eight mesocortical (dysgranular; $I d$ ) and 16 isocortical (granular; Ig) areas (adapted from Brockhaus 1940). E. Cytoarchitectonic division of the human insula posterior to central sulcus, with a subdivision of the granular and dysgranular insula into two and three areas, respectively (adapted with permission from Kurth, Eickhoff, et al. 2010). F. Cytoarchitectonic division of the rhesus macaque (Macaca mulata) insula into one agranular (Ia), one dysgranular (Id) and one granular (Ig) sector (adapted from Mesulam and Mufson 1982). G. A similar view of the cytoarchitectonic map shown in panel E, overlaid with a hodological division into one anteroventral "limbic" territory and one posterodorsal "auditory-somesthetic-skeletomotor" territory (adapted with permission from Mesulam and Mufson 1982). The separation between the two territories crosses the middle of the dysgranular sector (thick dashed line in Id). $\mathbf{H}$. Cytoarchitectonic division of the macaque insula into one vast "hypergranular" sector (G), two granular areas (Ig1 and 2), three dysgranular areas (Id1, 2 and 3) and two agranular areas (Ia1 and 2) (adapted with permission from Gallay et al. 2012). Note that the parcellation of the insula in the same subject (Mk4) but using other histological or immunohistochemical stains did not coincide with the cytoarchitectonic areas and with each other (see Gallay et al. 2012). I. Cyto- and myeloarchitectonic subdivision of the three classical sectors of the macaque insula into four granular areas (Idfa, Idfp, Igd, Igv), four dysgranular areas (Idd, Idm, Idv, Ivfp), and seven agranular areas (Ial, Iai, Iam, Iapl, Iap, Iapm, Ivfa) (adapted from Evrard et al. 2014). Three of the dysgranular 
areas $(I d d, I d m$ and $I d v)$ and one of the agranular areas (Iap) were further subdivided in 2 to 5 subareas. In all panels, top is dorsal and left is anterior. The different degrees of cytoarchitectonic granularity are represented with different tones of gray (black, "hypergranular"; dark gray, granular; middle gray, dysgranular; light gray, agranular). For the abbreviations in A to G, see original publications. For the abbreviations in $\mathrm{H}$, see the present abbreviations list. (adapted with permission from Evrard et al., 2014).

Figure 2. A-E. Drawings of the sites of injection of neuronal tracers in coronal sections of the right hemisphere of the cerebral cortex in five rhesus macaque monkeys. Each injection site is represented by a black core surrounded, in some cases, by a gray halo of diffusion. The five different cases are laid out from the most posterior (OM85) to the most anterior (OM69) injection. A. Injection site of lucifer yellow (LY) in area 24b' in OM85. B. Injection site of biotin dextran amine (BDA) in area 131 in OM42. C. Injection site of BDA in area 111 in case OM27. D. Injection site of BDA in area 12m in case OM30. E. Injection of LY in area12r (and 45/46v, not shown) in OM69. Medial is left; dorsal is top. Scale bar $=5 \mathrm{~mm}$. See the abbreviation list. F. Photomicrograph of representative retrogradely labeled pyramidal neurons in the insular cortex in case OM85 with an injection of lucifer yellow in area 24b'. The triple, double and single arrowheads point to the labeled perikarya, one of the basal dendrites and the apical dendrite, respectively. The arrow points to the unlabeled cell nucleus. G. Photomicrograph of representative anterogradely labeled axon terminals in the insular cortex in case OM85. The arrows point to examples of labeled varicosities localized along the axon fiber. F'-G'. Illustration of the labeling charting procedure. Scale bar $=10 \mu \mathrm{m}$.

Figure 3. Flat map representations of the distribution of anterograde (shades of red) and retrograde (dark dots) labeling across the distinct architectonic areas and subareas of the insular cortex in five rhesus macaque monkeys with injections of tracers in different prefrontal or cingulate cortical areas. Panels A to $\mathrm{C}$ illustrate the method used to re-create the flat map of the insula shown in panel D for case OM85. The same principle was applied for all flat maps (Evrard et al. 2014). A. Original drawing of anterograde (thin lines) and retrograde (dots) labeling in one coronal section of the insular cortex in case OM85. B. Red-shades color-coding of three different densities of anterogradely labeled terminals (weak, moderate, dense). C. Transfer of the color-coding and 
overall number of retrogradely labeled neurons onto a flattened representation of the areas and labeling of the section. D-H. Flat map representations of the distribution of anterograde and/or retrograde labeling in the insula in cases OM85 (D), OM42 (E), OM27 (F), OM30 (G) and OM69 (H). In panel D, the frontoparietal (FPO) and temporal (TO) opercula are represented with a pale gray. In panels E-H, the representation of FPO and TO was omitted to avoid cluttering the figure. In all flat maps, the thick lines represent the superior and inferior limiting sulci (SLS and ILS). In all cases, the coronal sections were aligned using SLS, which is therefore represented by the straight line. The thinner plain contours delineate architectonic areas. The dashed contours delineate subareas within an area. The vertical lines crossing the maps indicate the anteroposterior level of the coronal sections shown in Figures 4 to 13 to illustrate the overlap of architectonic and hodological entities (see text).

Figure 4. Composite showing the spatial overlap of patches of anterograde and retrograde labeling with specific cytoarchitectonic subareas of the insular cortex in case OM85 with an injection of lucifer yellow (LY) in cingulate area 24b'. A. Low magnification photomicrograph of a coronal section of the insula immunoreacted to visualize LY. Two patches of labeling are readily visible at low magnification. B. Plot of individual anterograde labeled terminals and retrogradely labeled perikarya made at high magnification in the same section as shown in panel A. Darkly and moderately stained perikarya are represented with black and gray dots, respectively. C. Low magnification photomicrograph of an adjacent Nissl-stained section showing the cytoarchitectonic boundaries between areas (arrowheads) and subareas (ticks). The same boundaries were transposed to the photomicrograph in panel B. Top is dorsal; left is medial. Scale $=1 \mathrm{~mm}$.

Figure 5. High magnification photomicrographs of the (A) dorsal, (B) middle and (C) ventral parts of the Nissl-stained section of the insula shown in panel $\mathrm{C}$ of Figure 4 in case OM85. The arrowheads and ticks mark the cytoarchitectonic boundaries between areas and subareas, respectively. The horizontal tick marks in panel B mark the separation of the cortical layers. Top is medial and left is dorsal. Scale bar $=1 \mathrm{~mm}$.

Figure 6. Composite showing the spatial overlap of patches of anterograde labeling with specific cytoarchitectonic subareas in the insular cortex in a case OM42 with an injection of biotin dextran 
amine (BDA) in the area 111 of the orbital prefrontal cortex (OPFC). A-D. Overlay of the charts of BDA-positive axon terminals on low-magnification photomicrographs of the corresponding BDA-stained sections of the insula across four coronal levels. The localization of the cytoarchitectonic boundaries between areas (arrowheads) and subareas (ticks) is shown for the subareas where anterograde labeling occurred. The distance of each coronal section from the level of the anterior commissure is noted in millimeters, in the bottom right corner of each panel (4500 $\mathrm{mm}$ in $\mathrm{A}$ to $0 \mathrm{~mm}$ in $\mathrm{D}$ ). A'-D'. Overlay of the demarcation of the cytoarchitectonic boundaries of the insula on low-magnification photomicrographs of Nissl-stained sections directly adjacent to the BDA-stained sections shown in panels A-D. All areas and subareas are noted. (See abbreviations list.) High-magnification photomicrographs of selected subareas are shown in Figures 9, 10 and 11, allowing clear recognition of the cytoarchitectonic boundaries. (See text.) The asterisks mark the position of blood vessels used as landmarks across adjacent BDA- and Nissl-stained sections. Scale $=1 \mathrm{~mm}$.

Figure 7. Overlay of the demarcation of cytoarchitectonic boundaries on high-magnification photomicrographs of four consecutive Nissl-stained sections, shown at low-magnification in Figure 6A'-D' in case OM42. The emphasis is on Idfp, Idd2 and Idd3 where anterograde labeling occurred. Areas are separated by an arrowhead. Subareas are separated by tick marks. (See text and abbreviation list.) Scale $=1 \mathrm{~mm}$.

Figure 8. Composite showing the spatial overlap of patches of anterograde labeling with specific cytoarchitectonic subareas in the insular cortex in a macaque monkey (OM27) with an injection of biotin dextran amine (BDA) in the area 111 of the orbital prefrontal cortex (OPFC). A-D. Overlay of the charts of BDA-positive axon terminals on low-magnification photomicrographs of the corresponding BDA-stained sections of the insula across four coronal levels. The localization of the cytoarchitectonic boundaries between areas (arrowheads) and subareas (ticks) is shown for the subareas where anterograde labeling occurred. The distance of each coronal section from the level of the anterior commissure is noted in millimeters, in the bottom right corner of each panel (4500 $\mathrm{mm}$ in $\mathrm{A}$ to $0 \mathrm{~mm}$ in $\mathrm{D}$ ). A'-D'. Overlay of the demarcation of the cytoarchitectonic boundaries of the insula on low-magnification photomicrographs of Nissl-stained sections directly adjacent to the BDA-stained sections shown in panels A-D. All areas and subareas are noted. (See 
abbreviations list.) High-magnification photomicrographs of selected subareas are shown in Figures 9, 10 and 11, allowing clear recognition of the cytoarchitectonic boundaries. (See text.) The asterisks mark the position of blood vessels used as landmarks across adjacent BDA- and Nissl-stained sections. Scale $=1 \mathrm{~mm}$.

Figure 9. Overlay of the demarcation of cytoarchitectonic boundaries on high-magnification photomicrographs of three Nissl-stained sections, shown at low-magnification in Figure 8A'-C' in case OM27. The emphasis is on subareas Idd1 and Idd2. Areas Idfp and Idd are separated by an arrowhead. Subareas 1, 2, 3 and 4 of Idd are separated by tick marks. (See text and abbreviation list.) Scale $=1 \mathrm{~mm}$.

Figure 10. Overlay of the demarcation of cytoarchitectonic boundaries on high-magnification photomicrographs of one Nissl-stained section, shown at low-magnification in Figure 8A' in case OM27. The emphasis is in on subareas Idd4. Subareas 3 and 4 of Idd are separated by tick marks. Areas Idd and Idm are separated by an arrowhead. (See text and abbreviation list.) Scale = $1 \mathrm{~mm}$.

Figure 11. Overlay of the demarcation of cytoarchitectonic boundaries on high-magnification photomicrographs of four Nissl-stained sections, shown at low-magnification in Figure 8A'-D' in case OM27. The emphasis is in on the subareas of areas Idm and Idv. Subareas are separated by tick marks. Areas are separated by arrowheads. (See text and abbreviation list.) Scale $=1 \mathrm{~mm}$.

Figure 12. Composite showing the spatial overlap of patches of anterograde labeling with specific cyto- and myelo-architectonic subareas in the insular cortex in a macaque monkey (OM30) with an injection of biotin dextran amine (BDA) in the area $12 \mathrm{~m}$ of the orbital prefrontal cortex (OPFC). A. Overlay of the charts of BDA-positive axon terminals on low-magnification photomicrographs of the corresponding BDA-stained sections of the insula. B and C. Overlay of the demarcation of, respectively, the cyto- and myelo-architectonic boundaries of the insula on low-magnification photomicrographs of Nissl- and Gallyas-stained sections directly adjacent to the BDA-stained section shown in panel A. All areas and subareas are noted. (See abbreviations list.) D and E. High-magnification view of the cyto- and myelo-architecture and boundaries in the dorsal half of 
the insula from panels B and C. In panel E, the outer band of Baillarger is indicated (obb). Scale = $1 \mathrm{~mm}$.

Figure 13. Composite showing the spatial overlap of patches of anterograde labeling with specific cyto- and myelo-architectonic subareas in the insular cortex in a macaque monkey (OM69) with an injection of lucifer yellow (LY) in the area 12r of the orbital prefrontal cortex (OPFC). A-B. Overlay of the charts of LY-positive axon terminals (A) and cell bodies (B) on low-magnification photomicrographs of the corresponding LY-stained sections of the insula. C-D. Overlay of the demarcation of the cyto- (C) and myelo- (D) architectonic boundaries of the insula on lowmagnification photomicrographs of Nissl- and Gallyas-stained sections directly adjacent to the BDA-stained section shown in panel A. E-F. High-magnification view of the cyto- and myeloarchitecture and boundaries in the middle of the insula from panels $\mathrm{C}$ and $\mathrm{D}$. In panel $\mathrm{F}$, the outer band of Baillarger is indicated (obb). (See abbreviations list.) Scale $=1 \mathrm{~mm}$.

Figure 14. A. Coronal photomicrographs showing patches of tritiated amino acid (TAA, left) and horseradish peroxidase (HRP, right) anterograde labeling in the insula with injections in the prefrontal and posterior parietal cortices (Adapted with permission from Selemon \& GoldmanRakic, 1988). B. Distribution of DY-labeled cells on selected coronal sections through the dorsal dysgranular insula after injections in the distal toe region of area $3 \mathrm{~b}$. Each dot represents the location of one retrogradely labeled cell (Adapted with permission from Burton et al., 1995). C. Flat map representation of "stripes" of retrograde labeling in the insula in 3 monkeys with injection of HRP in distinct parts of the brain. The injections were made in the frontoparietal operculum (7a), posterior parietal cortex (8a) and lateral prefrontal cortex (9a) (Adapted with permission from Mesulam and Mufson, 1982). 


\section{References}

Allen GV, Saper CB, Hurley KM, Cechetto DF. 1991. Organization of visceral and limbic connections in the insular cortex of the rat. The Journal of comparative neurology. 311:1-16.

Amunts K, Schleicher A, Zilles K. 2002. Architectonic Mapping of the Human Cerebral Cortex. In: Schuz A, Miller R, editors. Cortical Areas: Unity and Diversity London: Taylor \& Francis p 29-52.

An X, Bandler R, Ongur D, Price JL. 1998. Prefrontal cortical projections to longitudinal columns in the midbrain periaqueductal gray in macaque monkeys. Journal of Comparative Neurology. 401:455-479.

Bauernfeind AL, de Sousa AA, Avasthi T, Dobson SD, Raghanti MA, Lewandowski AH, Zilles K, Semendeferi K, Allman JM, Craig AD, Hof PR, Sherwood CC. 2013. A volumetric comparison of the insular cortex and its subregions in primates. Journal of human evolution. 64:263-279.

Borra E, Belmalih A, Calzavara R, Gerbella M, Murata A, Rozzi S, Luppino G. 2008. Cortical connections of the macaque anterior intraparietal (AIP) area. Cereb Cortex. 18:1094-1111.

Brockhaus H. 1940. Die Cyto- und Myeloarchitektonik des Cortex claustralis und des Claustrum beim Menschen. Journal für Psychologie und Neurologie. 49:249-348.

Brodmann K. 1909. Vergleichende Lokalizationlehre der Grosshirnrinde. Leipzig: Johann Ambrosius Barth.

Burton H, Jones EG. 1976. The posterior thalamic region and its cortical projection in New World and Old World monkeys. Journal of Comparative Neurology. 168:249-301.

Carmichael ST, Price JL. 1994. Architectonic subdivision of the orbital and medial prefrontal cortex in the macaque monkey. Journal of Comparative Neurology. 346:366-402.

Carmichael ST, Price JL. 1995. Sensory and premotor connections of the orbital and medial prefrontal cortex of macaque monkeys. Journal of Comparative Neurology. 363:642-664.

Cavada C, Huisman AM, Kuypers HG. 1984. Retrograde double labeling of neurons: the combined use of horseradish peroxidase and diamidino yellow dihydrochloride (DY X 2HCl) compared with true blue and DY X $2 \mathrm{HCl}$ in rat descending brainstem pathways. Brain research. 308:123136.

Cerliani L, D'Arceuil H, Thomas RM, Jbabdi S, Keysers C. 2010. Gradual variation of anatomical connectivity in the macaque insula revealed by probabilistic tractography. Proc Intl Soc Mag Reson Med. 18:1662.

Cerliani L, Thomas RM, Jbabdi S, Siero JC, Nanetti L, Crippa A, Gazzola V, D'Arceuil H, Keysers C. 2012. Probabilistic tractography recovers a rostrocaudal trajectory of connectivity variability in the human insular cortex. Hum Brain Mapping. 33:2005-2034.

Chikama M, McFarland NR, Amaral DG, Haber SN. 1997. Insular cortical projections to functional regions of the striatum correlate with cortical cytoarchitectonic organization in the primate. Journal of Neuroscience. 17:9686-9705.

Cipolloni PB, Pandya DN. 1999. Cortical connections of the frontoparietal opercular areas in the rhesus monkey. The Journal of comparative neurology. 403:431-458.

Craig AD. 2002. How do you feel? Interoception: the sense of the physiological condition of the body. Nature reviews Neuroscience. 3:655-666.

Craig AD. 2009. How do you feel--now? The anterior insula and human awareness. Nature reviews Neuroscience. 10:59-70. 
Deacon TW. 1992. Cortical connections of the inferior arcuate sulcus cortex in the macaque brain. Brain research. 573:8-26.

Deen B, Pitskel NB, Pelphrey KA. 2011. Three systems of insular functional connectivity identified with cluster analysis. Cerebral Cortex. 21:1498-1506.

Evrard HC. 2018. Von Economo and fork neurons in the monkey insula, implications for evolution of cognition. Current Opinion in Behavioral Sciences. 21:182-190.

Evrard HC, Craig AD. 2015. Insular Cortex. In. Brain Mapping: An Encyclopedic Reference Elsevier p 387-393.

Evrard HC, Logothetis NK, Craig AD. 2014. Modular architectonic organization of the insula in the macaque monkey. Journal of Comparative Neurology. 522:64-97.

Evrard HC. 2019. The Organization of the Primate Insular Cortex. Front Neuroanat.

Ferry AT, Ongur D, An X, Price JL. 2000. Prefrontal cortical projections to the striatum in macaque monkeys: evidence for an organization related to prefrontal networks. The Journal of comparative neurology. 425:447-470.

Fudge JL, Breitbart MA, Danish M, Pannoni V. 2005. Insular and gustatory inputs to the caudal ventral striatum in primates. The Journal of comparative neurology. 490:101-118.

Gabbott PL, Warner TA, Jays PR, Bacon SJ. 2003. Areal and synaptic interconnectivity of prelimbic (area 32), infralimbic (area 25) and insular cortices in the rat. Brain research. 993:5971.

Gallay DS, Gallay MN, Jeanmonod D, Rouiller EM, Morel A. 2012. The insula of Reil revisited: multiarchitectonic organization in macaque monkeys. Cerebral Cortex. 22:175-190.

Goldman-Rakic PS, Schwartz ML. 1982. Interdigitation of contralateral and ipsilateral columnar projections to frontal association cortex in primates. Science. 216:755-757.

Goodkind M, Eickhoff SB, Oathes DJ, Jiang Y, Chang A, Jones-Hagata LB, Ortega BN, Zaiko YV, Roach EL, Korgaonkar MS, Grieve SM, Galatzer-Levy I, Fox PT, Etkin A. 2015. Identification of a common neurobiological substrate for mental illness. JAMA psychiatry. 72:305-315.

Gu X, Liu X, Van Dam NT, Hof PR, Fan J. 2013. Cognition-emotion integration in the anterior insular cortex. Cereb Cortex. 23:20-27.

Hendrickson ae. 1985. Dots, stripes and columns in monkey visual cortex. Trends in Neuroscience. 8:406-410.

Horn FM, Evrard HC editors. Multiple areal distribution of the von Economo and fork neurons in the human anterior insular cortex, 49th Annual Meeting of the Society for Neuroscience; 2018; San Diego.

Horn FM, Hertl M, Evrard HC. submitted. Elemental localization of the von Econmo neuron in the macaque monkey.

Horn FM, Hertl M, Logothetis NK, Evrard HC editors. Elemental localization of the von Economo neuron in the macaque monkey insula. , 12th National Congress of the Belgian Society for Neuroscience; 2017:Front. Neurosci.

Horton JC, Adams DL. 2005. The cortical column: a structure without a function. Philosophical transactions of the Royal Society of London Series B, Biological sciences. 360:837-862.

Jasmin L, Burkey AR, Granato A, Ohara PT. 2004. Rostral agranular insular cortex and pain areas of the central nervous system: a tract-tracing study in the rat. The Journal of comparative neurology. 468:425-440. 
Jones EG, Burton H. 1976. Areal differences in the laminar distribution of thalamic afferents in cortical fields of the insular, parietal and temporal regions of primates. The Journal of comparative neurology. 168:197-247.

Kaas JH. 2002. Cortical Areas and Patterns of Cortico-Cortical Connections. London: Taylor \& Francis.

Kaas JH. 2004. Evolution of somatosensory and motor cortex in primates. Anat Rec A Discov Mol Cell Evol Biol. 281:1148-1156.

Kaas JH. 2012. Evolution of columns, modules, and domains in the neocortex of primates. Proceedings of the National Academy of Sciences of the United States of America. 109 Suppl 1:10655-10660.

Kelly C, Toro R, Di Martino A, Cox CL, Bellec P, Castellanos FX, Milham MP. 2012. A convergent functional architecture of the insula emerges across imaging modalities. NeuroImage.

Kurth F, Eickhoff SB, Schleicher A, Hoemke L, Zilles K, Amunts K. 2010. Cytoarchitecture and probabilistic maps of the human posterior insular cortex. Cerebral Cortex. 20:1448-1461.

Kurth F, Zilles K, Fox PT, Laird AR, Eickhoff SB. 2010. A link between the systems: functional differentiation and integration within the human insula revealed by meta-analysis. Brain, Structure \& Function. 214:519-534.

Lavenex P, Suzuki WA, Amaral DG. 2002. Perirhinal and parahippocampal cortices of the macaque monkey: projections to the neocortex. The Journal of comparative neurology. 447:394-420.

Levitt JB, Lewis DA, Yoshioka T, Lund JS. 1993. Topography of pyramidal neuron intrinsic connections in macaque monkey prefrontal cortex (areas 9 and 46). The Journal of comparative neurology. 338:360-376.

Lewis JW, Van Essen DC. 2000. Mapping of architectonic subdivisions in the macaque monkey, with emphasis on parieto-occipital cortex. The Journal of comparative neurology. 428:79-111.

Lund JS, Yoshioka T, Levitt JB. 1993. Comparison of intrinsic connectivity in different areas of macaque monkey cerebral cortex. Cereb Cortex. 3:148-162.

Matelli M, Camarda R, Glickstein M, Rizzolatti G. 1986. Afferent and efferent projections of the inferior area 6 in the macaque monkey. The Journal of comparative neurology. 251:281-298.

Mesulam MM, Mufson EJ. 1982. Insula of the old world monkey. I. Architectonics in the insuloorbito-temporal component of the paralimbic brain. Journal of Comparative Neurology. 212:122.

Mesulam MM, Mufson EJ. 1982. Insula of the old world monkey. III: Efferent cortical output and comments on function. Journal of Comparative Neurology. 212:38-52.

Mesulam MM, Mufson EJ. 1985. The insula of reil in man and monkey. In: Peters A, Jones EG, editors. Cerebral Cortex New York: Plenum Press p 179-226.

Morel A, Gallay MN, Baechler A, Wyss M, Gallay DS. 2013. The human insula: Architectonic organization and postmortem MRI registration. Neuroscience. 236:117-135.

Mountcastle VB. 1997. The columnar organization of the neocortex. Brain : a journal of neurology. 120 ( Pt 4):701-722.

Mountcastle VB, Davies PW, Berman AL. 1957. Response properties of neurons of cat's somatic sensory cortex to peripheral stimuli. Journal of neurophysiology. 20:374-407.

Mufson EJ, Mesulam MM. 1982. Insula of the old world monkey. II: Afferent cortical input and comments on the claustrum. Journal of Comparative Neurology. 212:23-37. 
Mufson EJ, Sobreviela T, Kordower JH. 1997. Chemical neuroanatomy of the primate insula cortex: relationship to cytoarchiteconics, connectivity, function and neurodegeneration. In: VBloom FE, Bjorklund A, Hokfelt T, editors. Handbook of Chemical Neuroanatomy New York: Elsevier.

Naidich TP, Kang E, Fatterpekar GM, Delman BN, Gultekin SH, Wolfe D, Ortiz O, Yousry I, Weismann M, Yousry TA. 2004. The insula: anatomic study and MR imaging display at $1.5 \mathrm{~T}$. AJNR American journal of neuroradiology. 25:222-232.

Pandya DN, Van Hoesen GW, Mesulam MM. 1981. Efferent connections of the cingulate gyrus in the rhesus monkey. Experimental brain research. 42:319-330.

Preuss TM, Goldman-Rakic PS. 1989. Connections of the ventral granular frontal cortex of macaques with perisylvian premotor and somatosensory areas: anatomical evidence for somatic representation in primate frontal association cortex. The Journal of comparative neurology. 282:293-316.

Price JL. 2007. Definition of the orbital cortex in relation to specific connections with limbic and visceral structures and other cortical regions. Ann N Y Acad Sci. 1121:54-71.

Purves D, Riddle DR, LaMantia AS. 1992. Iterated patterns of brain circuitry (or how the cortex gets its spots). Trends in neurosciences. 15:362-368.

Qi X-H, Preuss TM, Kaas JH. 2008. Somatosensory Areas of the Cerebral Cortex: Architectonic Characteristics and Modular Organization. In: Gardner EP, Kaas JH, editors. The Senses: A Comprehensive Reference London: Elsevier p 143-170.

Reil JC. 1809. Die sylvische Grube. ArchPhysiol(Halle). 9:195-208.

Roberts TS, Akert K. 1963. Insular and opercular cortex and its thalamic projection in Macaca mulatta. Schweizer Archiv fur Neurologie, Neurochirurgie und Psychiatrie = Archives suisses de neurologie, neurochirurgie et de psychiatrie. 92:1-43.

Rose M. 1928. Die Inselrinde des Menschen und der Tiere. Journal of Physchology and Neurology. 37:467-624.

Saleem KS, Kondo H, Price JL. 2008. Complementary circuits connecting the orbital and medial prefrontal networks with the temporal, insular, and opercular cortex in the macaque monkey. Journal of Comparative Neurology. 506:659-693.

Salomon R, Ronchi R, Donz J, Bello-Ruiz J, Herbelin B, Martet R, Faivre N, Schaller K, Blanke O. 2016. The Insula Mediates Access to Awareness of Visual Stimuli Presented Synchronously to the Heartbeat. The Journal of neuroscience : the official journal of the Society for Neuroscience. 36:5115-5127.

Sanides F. 1968. The architecture of the cortical taste nerve areas in squirrel monkey (Saimiri sciureus) and their relationships to insular, sensorimotor and prefrontal regions. Brain research. 8:97-124.

Selemon LD, Goldman-Rakic PS. 1988. Common cortical and subcortical targets of the dorsolateral prefrontal and posterior parietal cortices in the rhesus monkey: evidence for a distributed neural network subserving spatially guided behavior. The Journal of neuroscience : the official journal of the Society for Neuroscience. 8:4049-4068.

Shi CJ, Cassell MD. 1998. Cortical, thalamic, and amygdaloid connections of the anterior and posterior insular cortices. The Journal of comparative neurology. 399:440-468.

Stefanacci L, Amaral DG. 2000. Topographic organization of cortical inputs to the lateral nucleus of the macaque monkey amygdala: a retrograde tracing study. Journal of Comparative Neurology. 421:52-79.

Swindale NV. 1990. Is the cerebral cortex modular? Trends in neurosciences. 13:487-492. 
Vogt C, Vogt O. 1919. Allgemeine Ergebnisse unserer Hirnforschung. Journal of Physchology and Neurology. 25:279-461.

Vogt O. 1911. Nouvelle contribution à l'étude de la myéloarchitecture de l'écorce cérébrale. XXe Congrès des médecins aliénistes et Neurologistes de France et des pays de langue française.311 . 


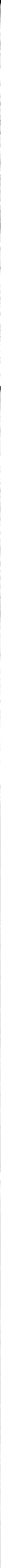


A

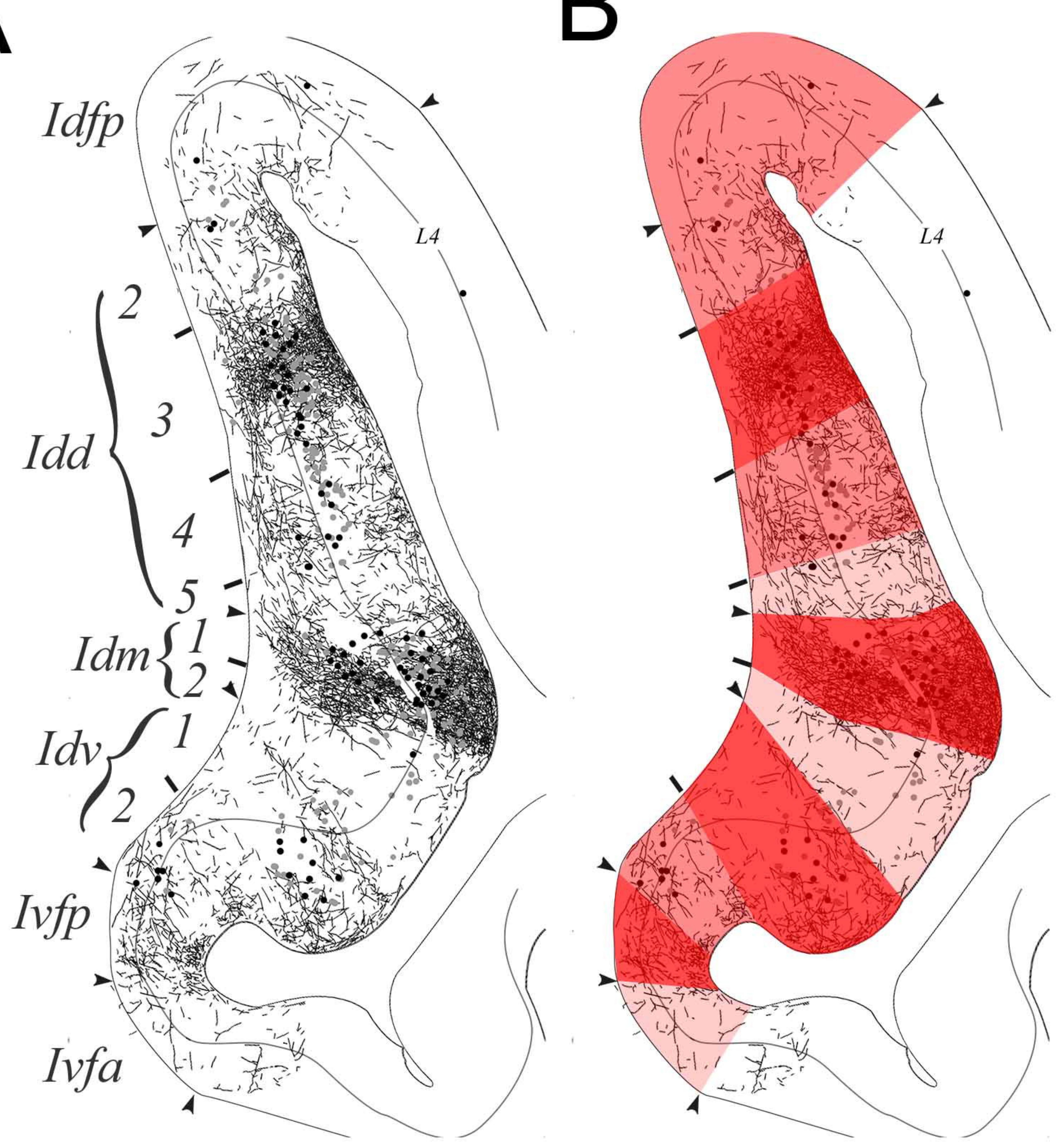

E

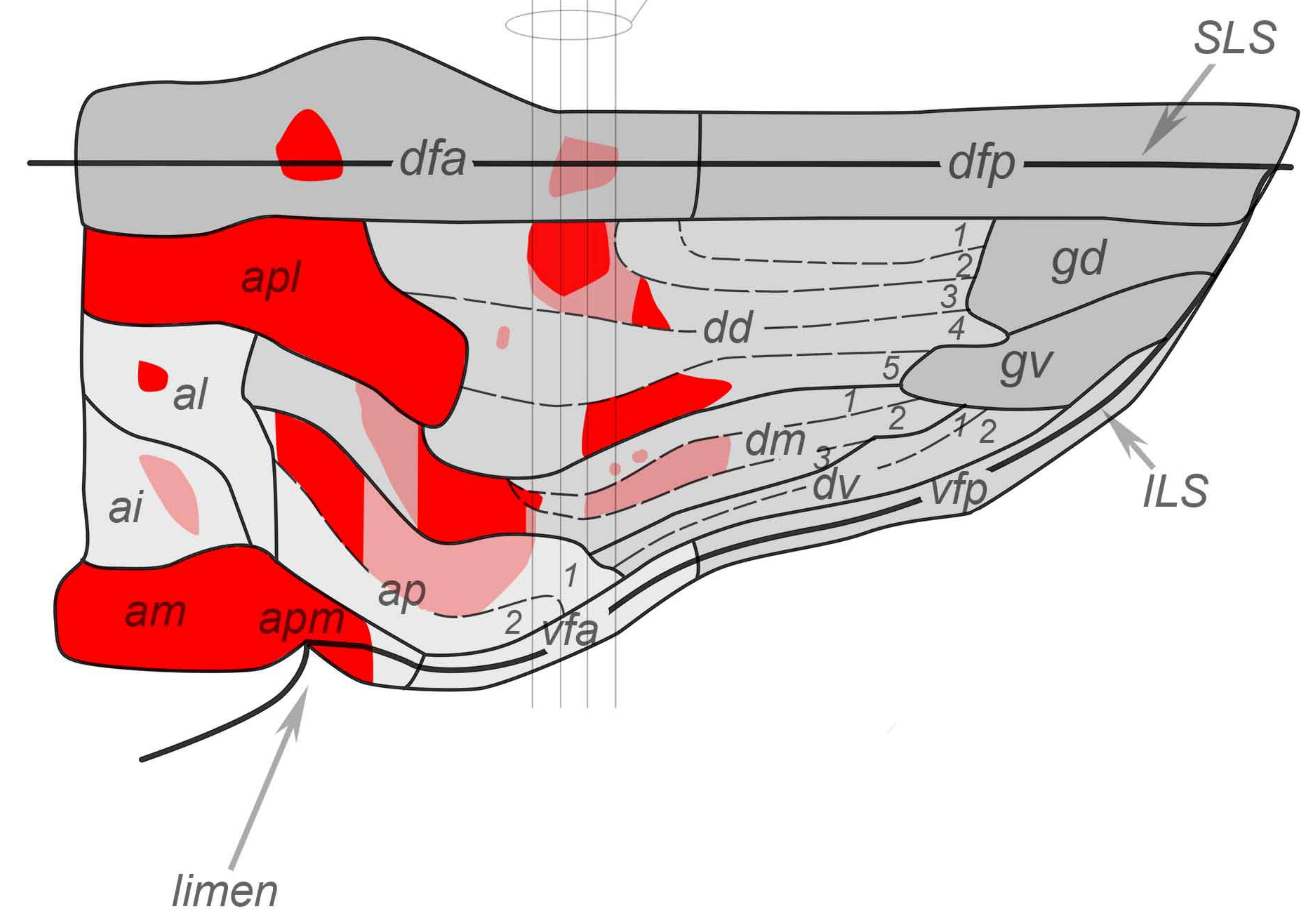

G

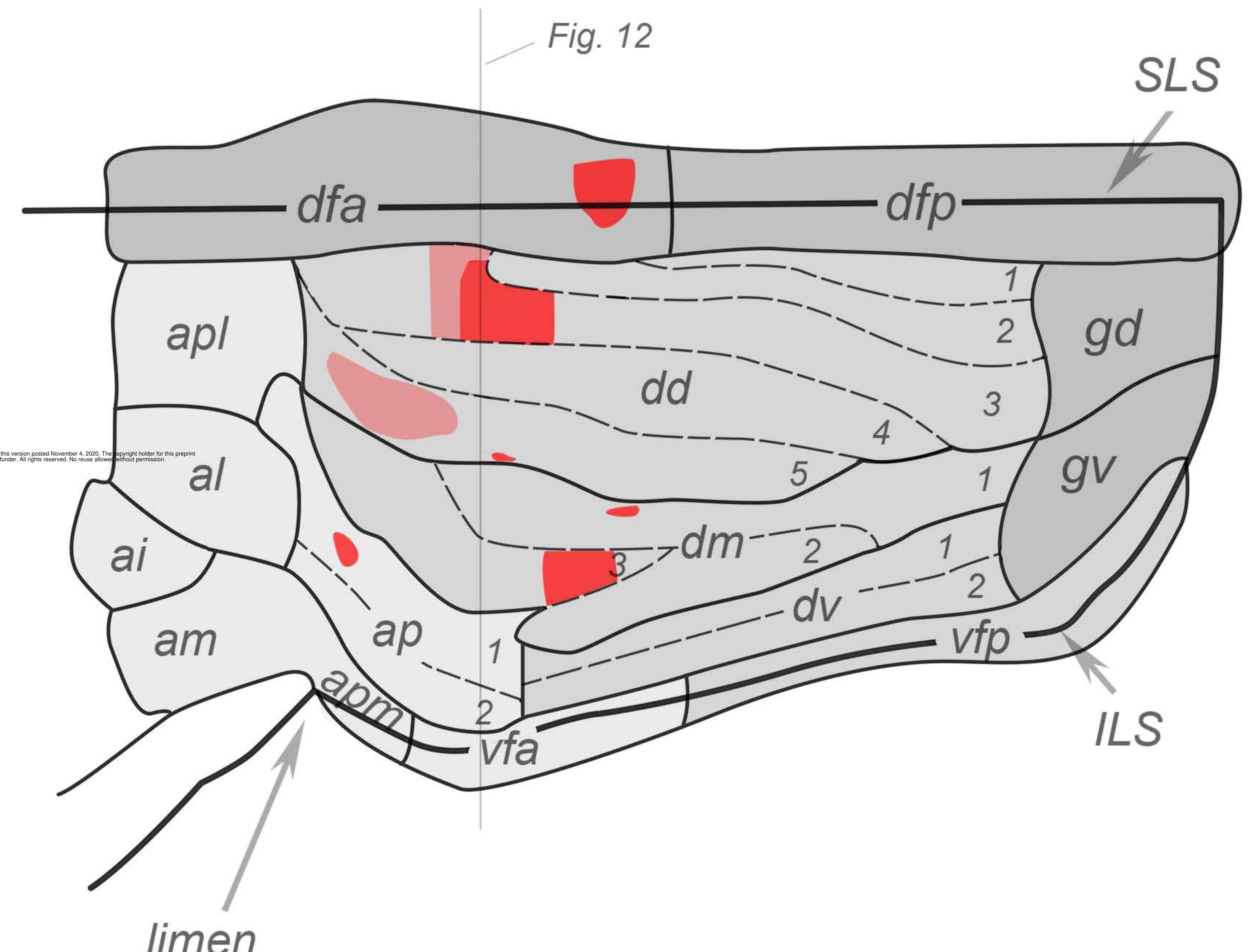



F



SLS

$\mathrm{H}$

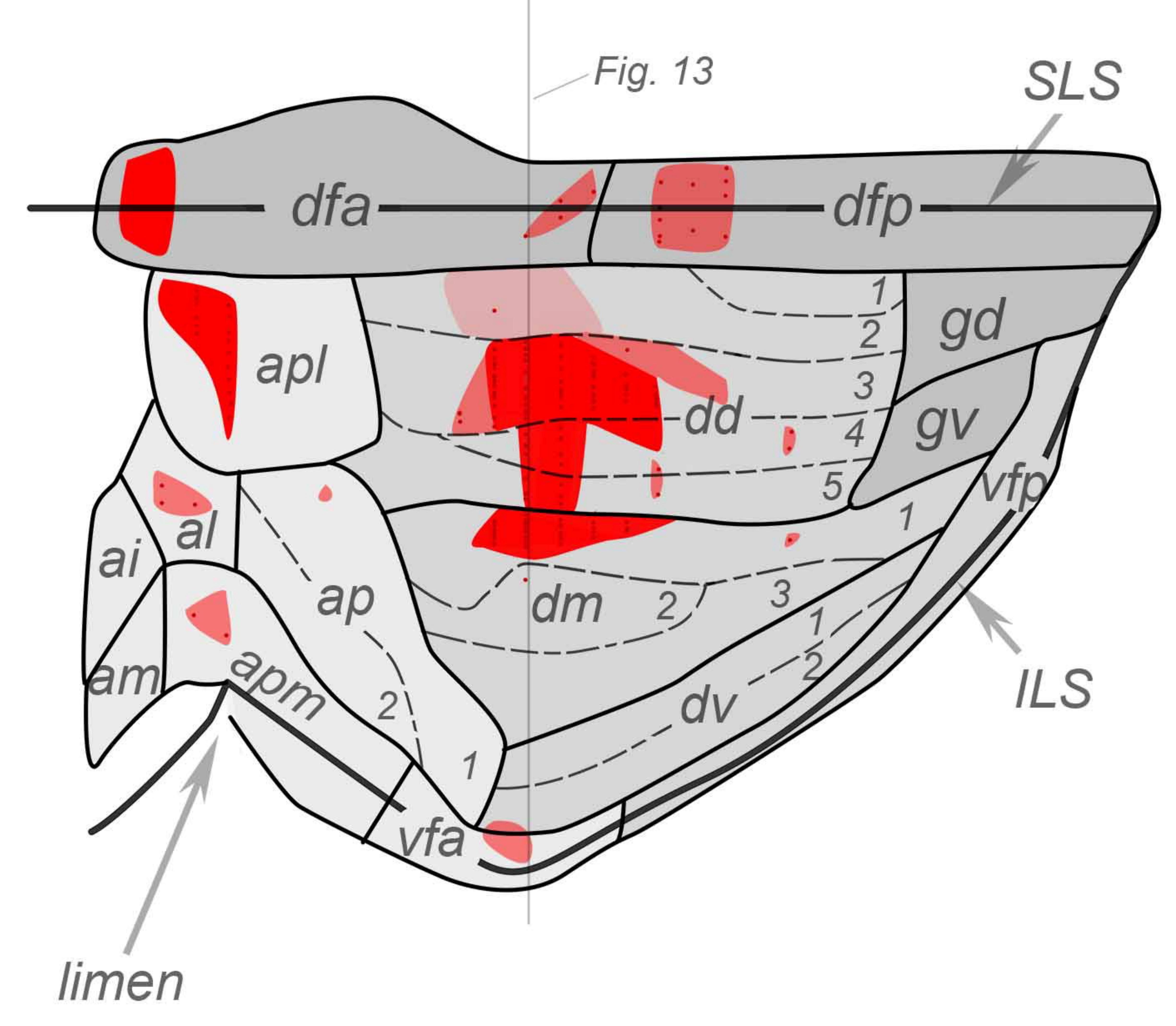




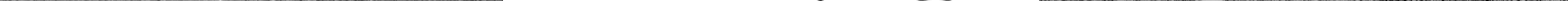




\section{$I d f p$}

\section{Idd2 I Idd}

\section{Idd 4}

B

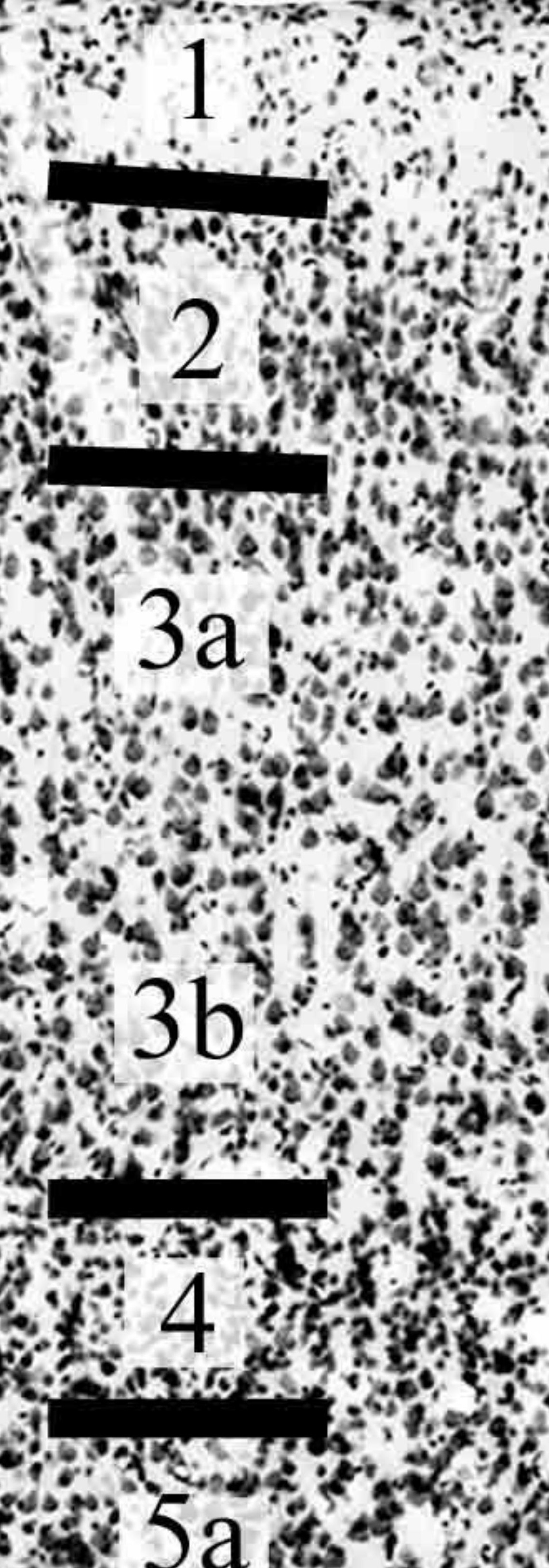

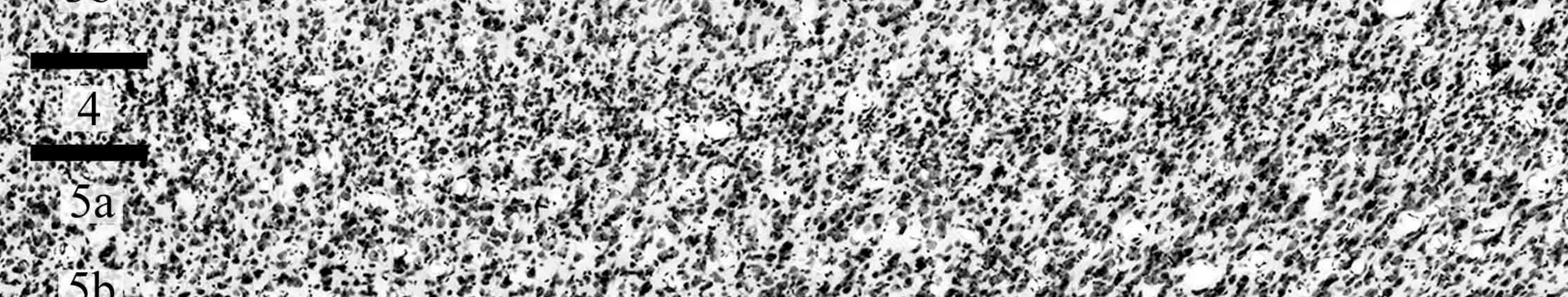

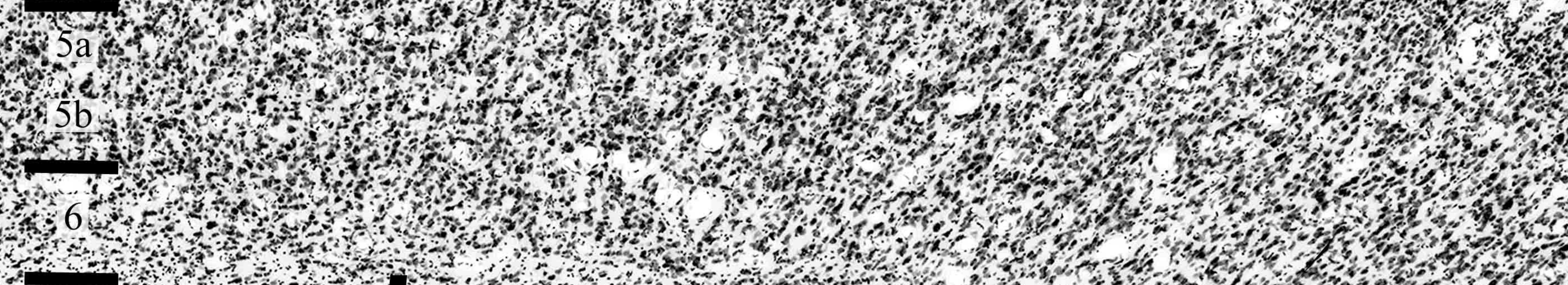

Idd 4

Idd 5

\section{$I d m I$}

\section{Idm 2}

dety

is $x$

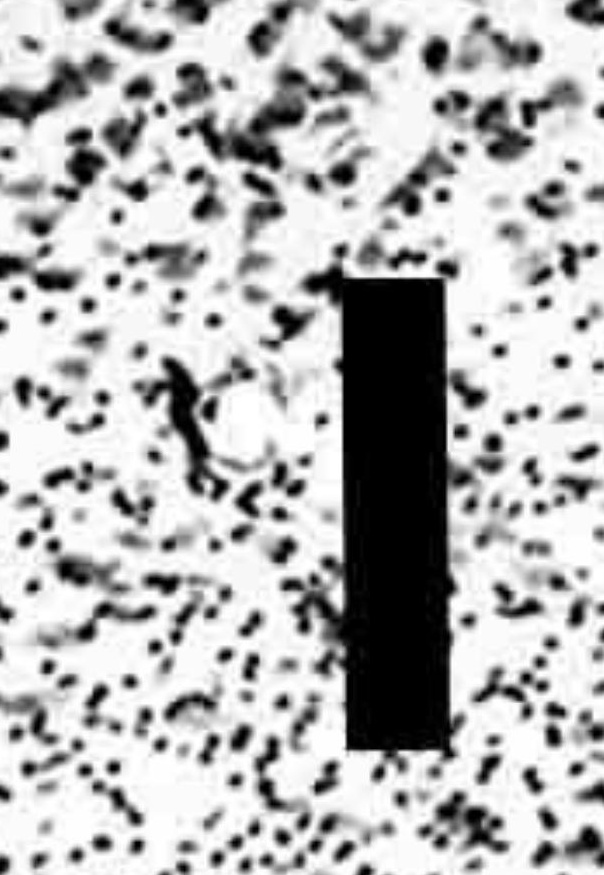


A

$I d f p$

B

$I d f p$

C

$I d f p$

$D$

$I d f p$
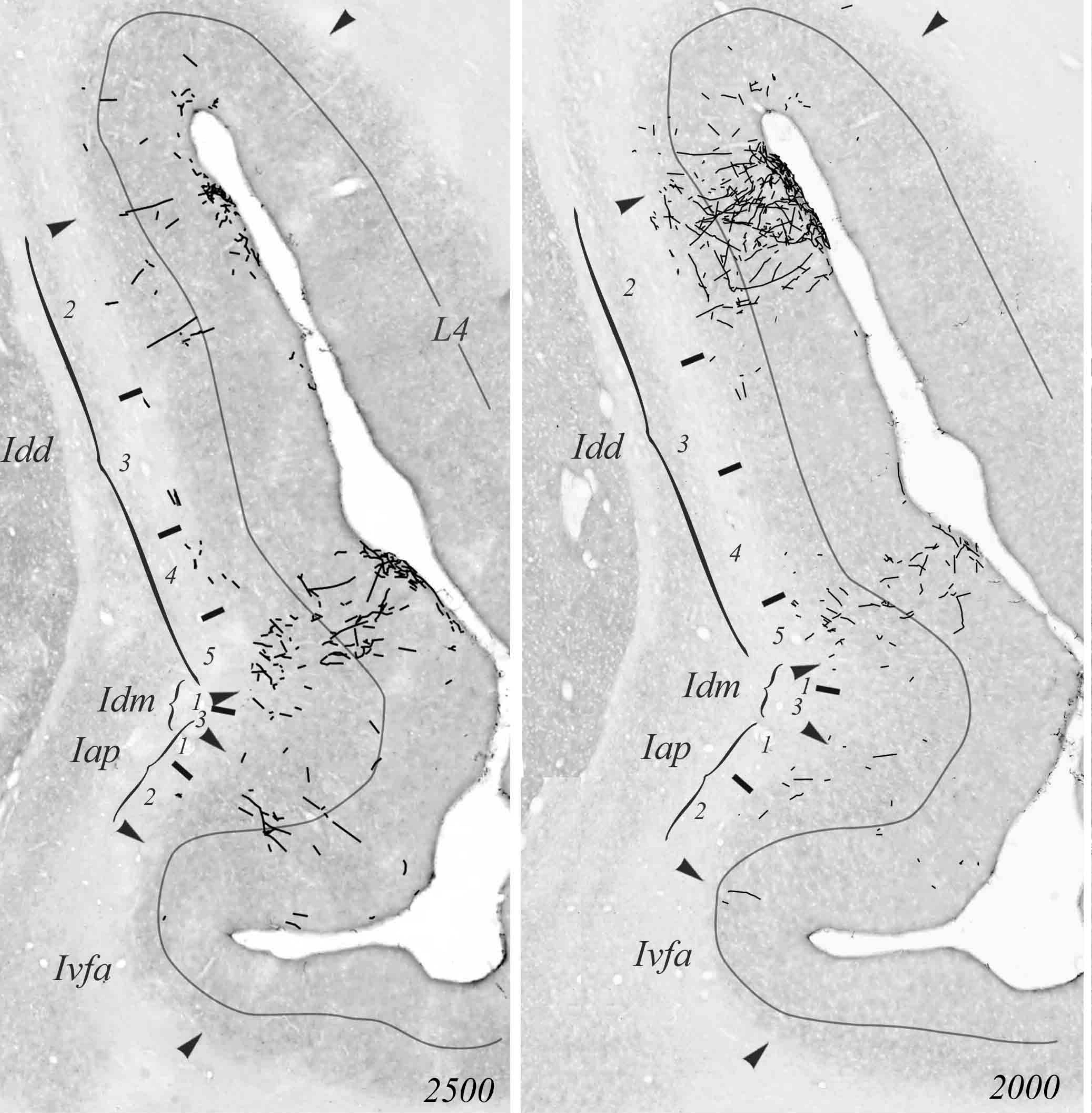

A

$I d f p$

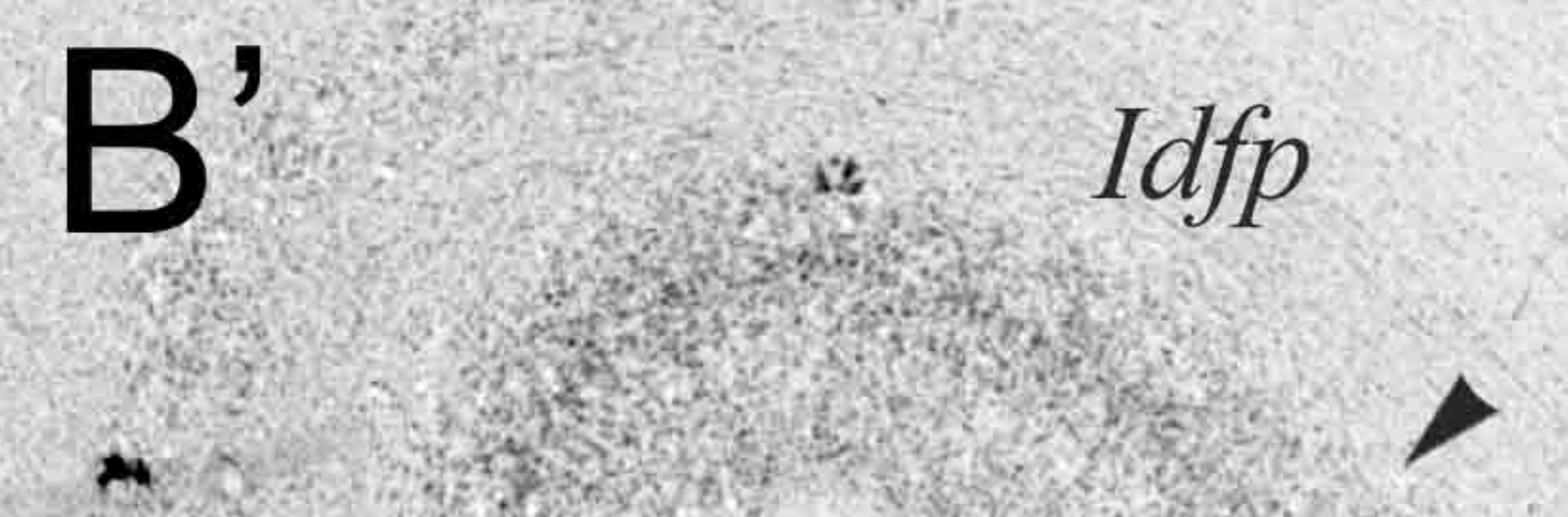

$I d f p$

(4. 1

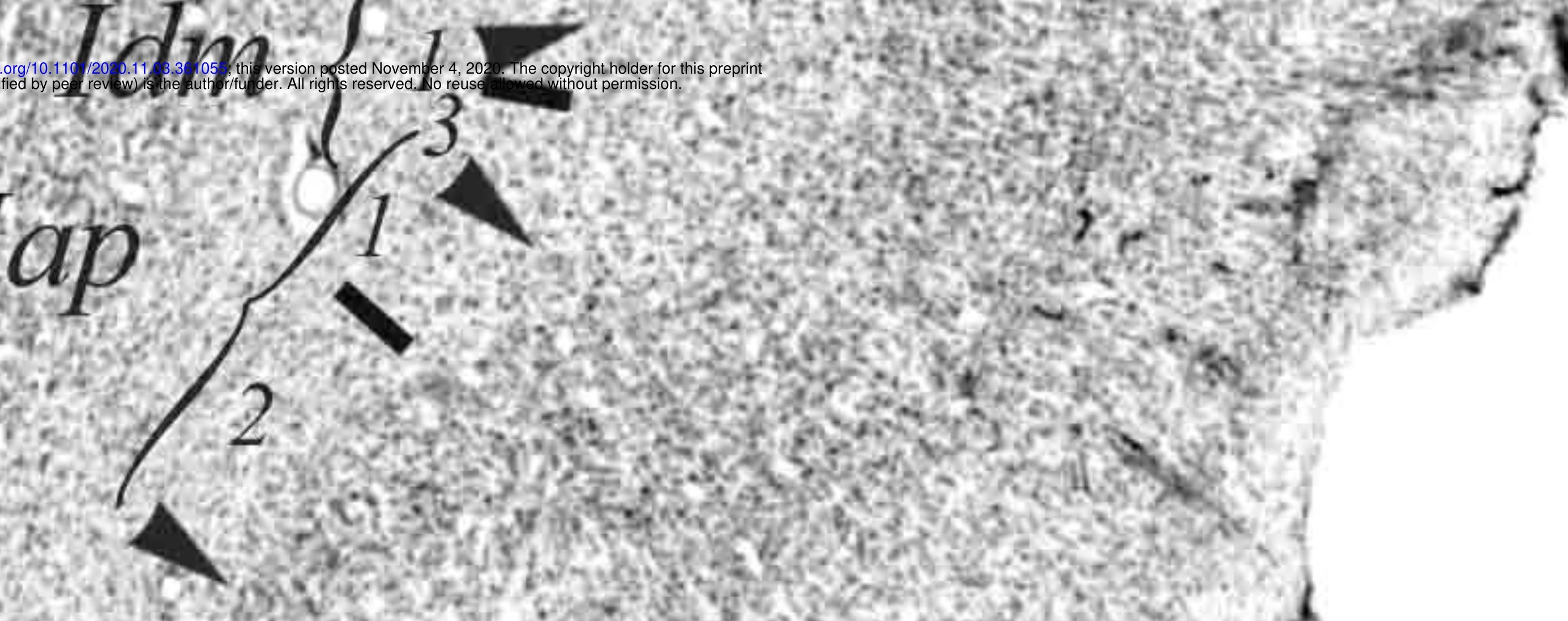

lifa
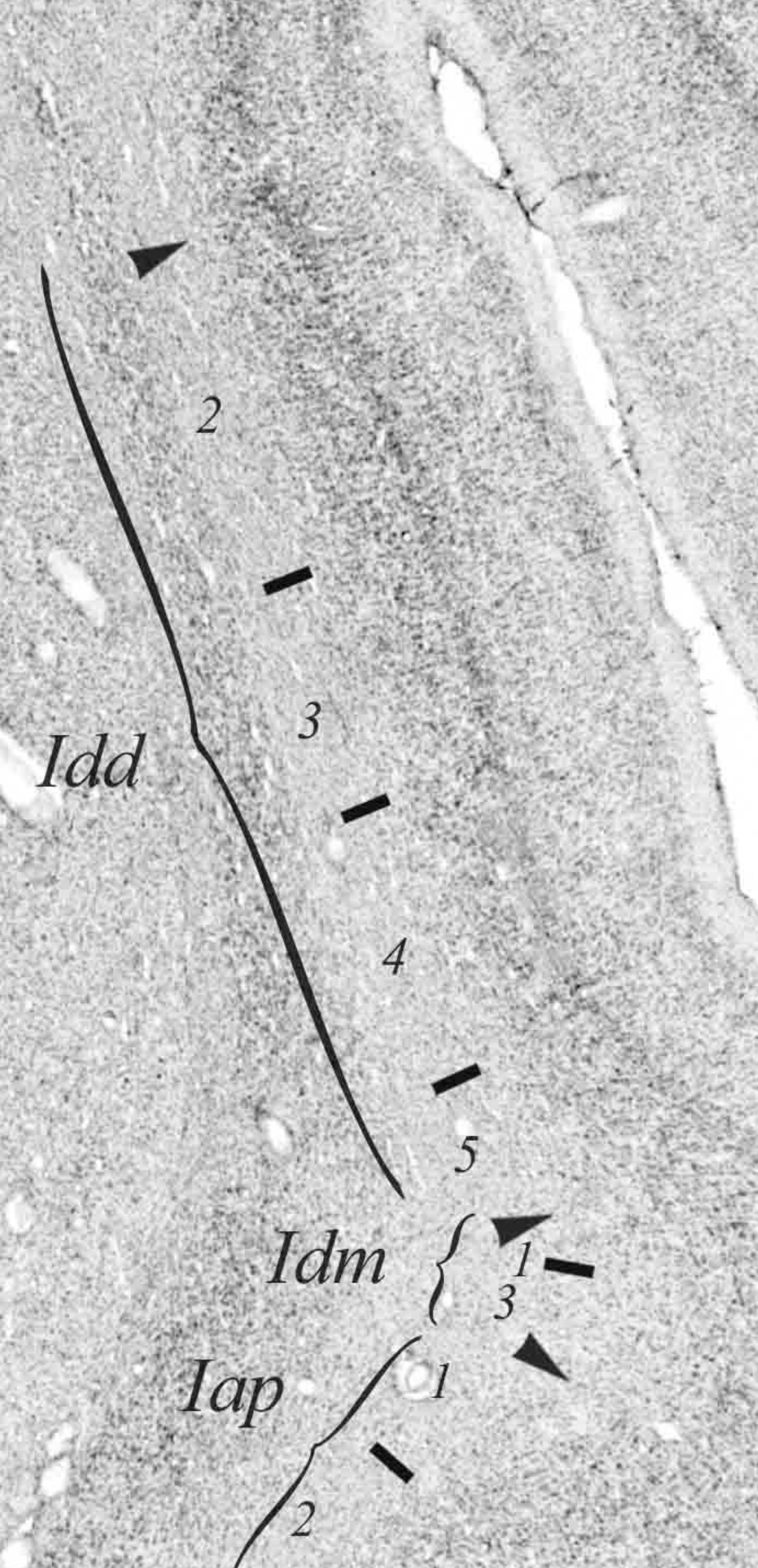

Ivfa
C

1500

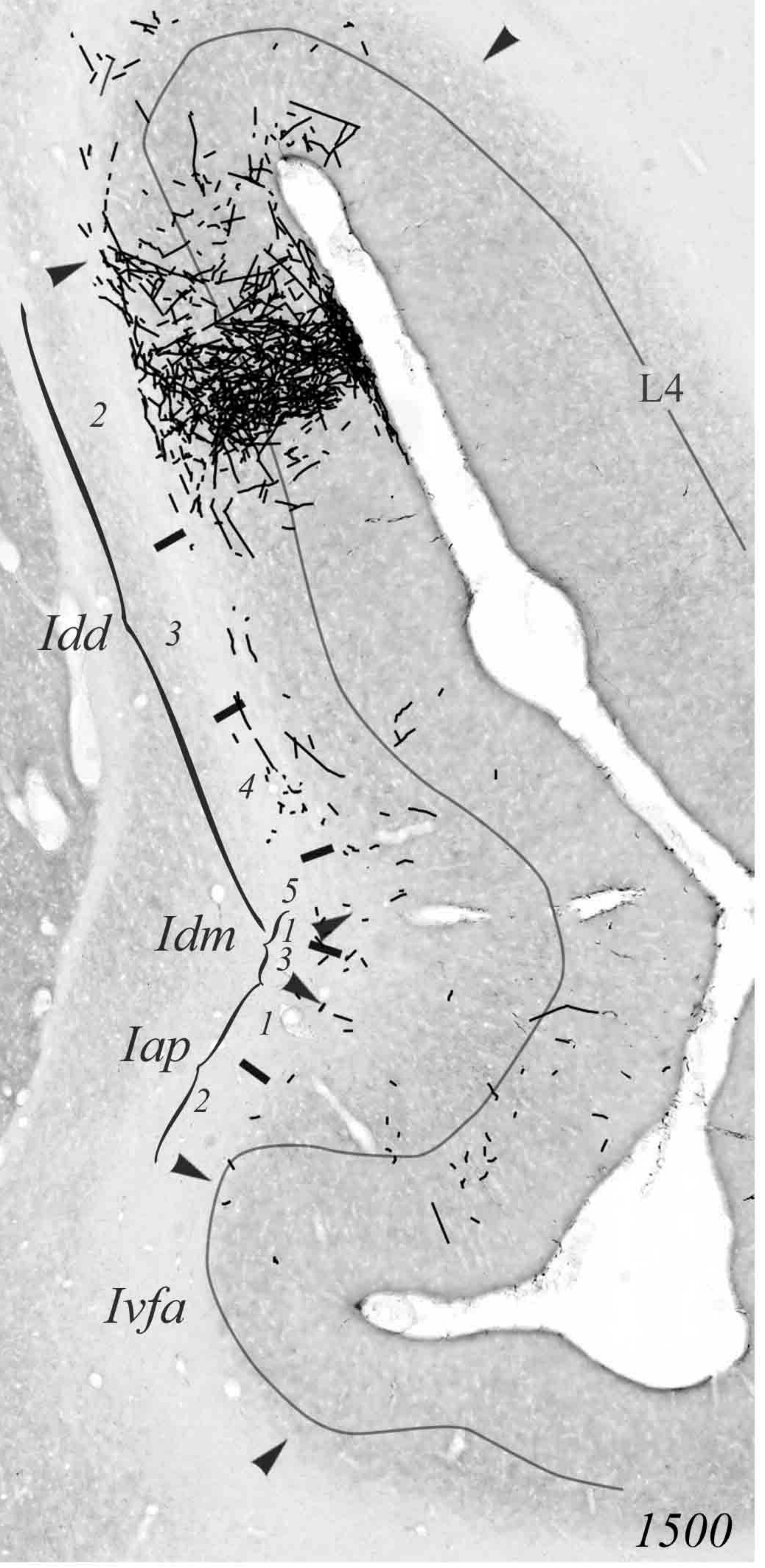

$I d f p$



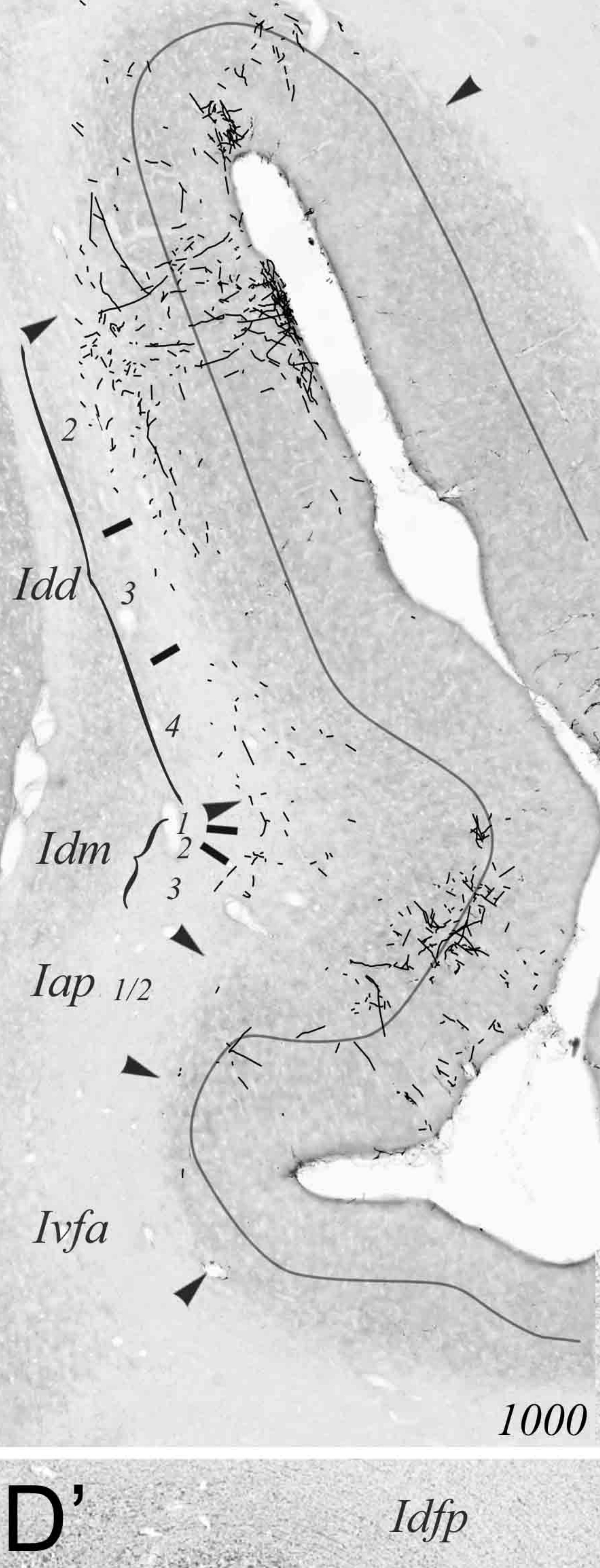

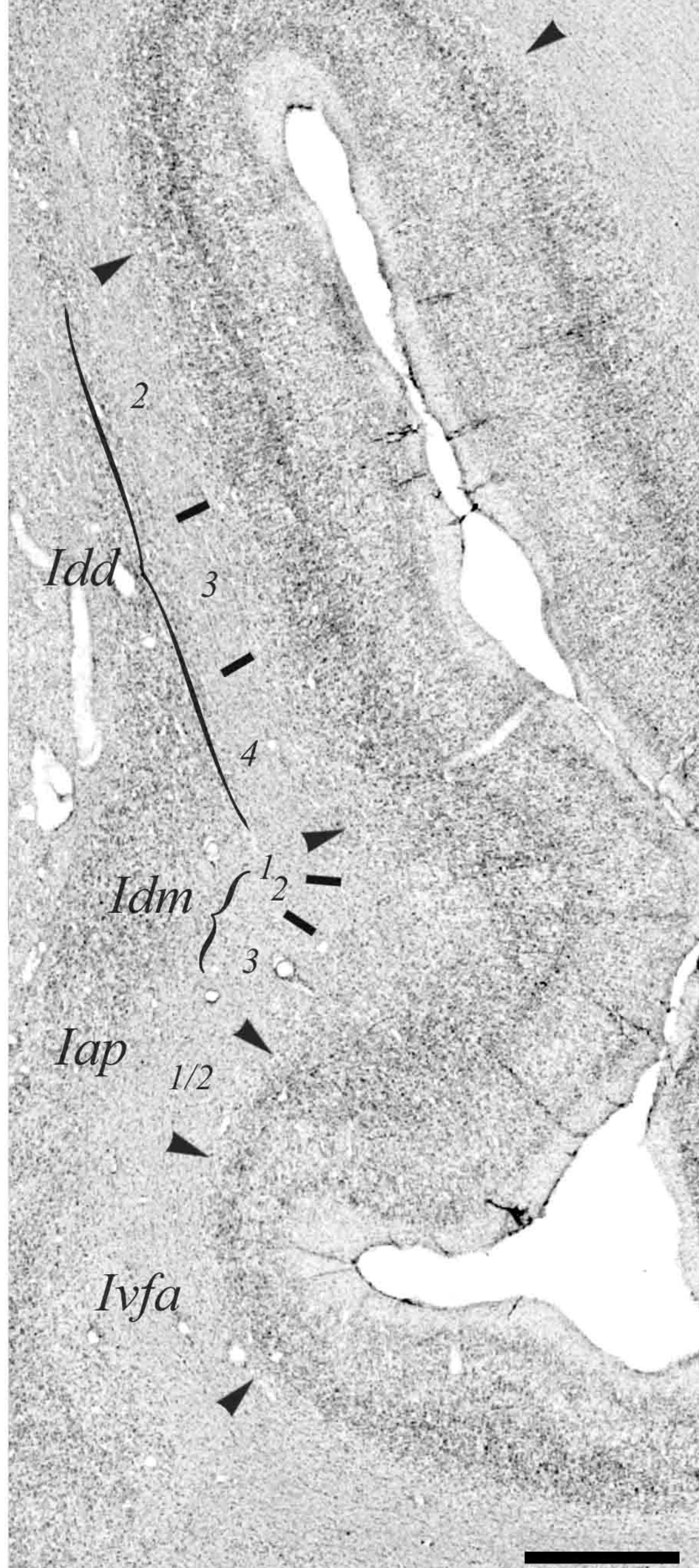

Ivfa 
.

(2)

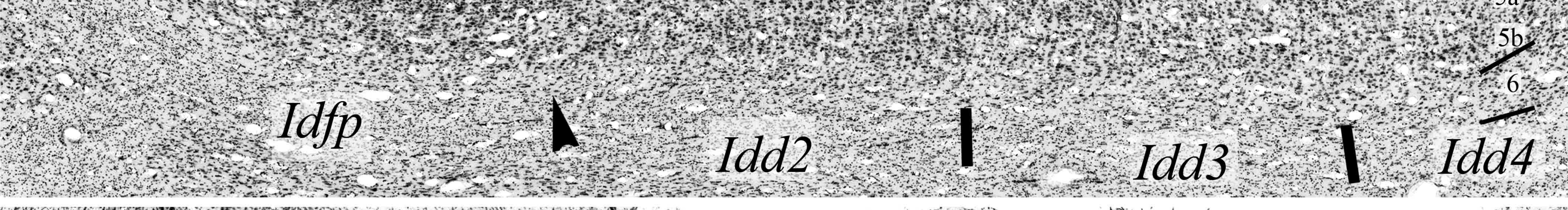

B

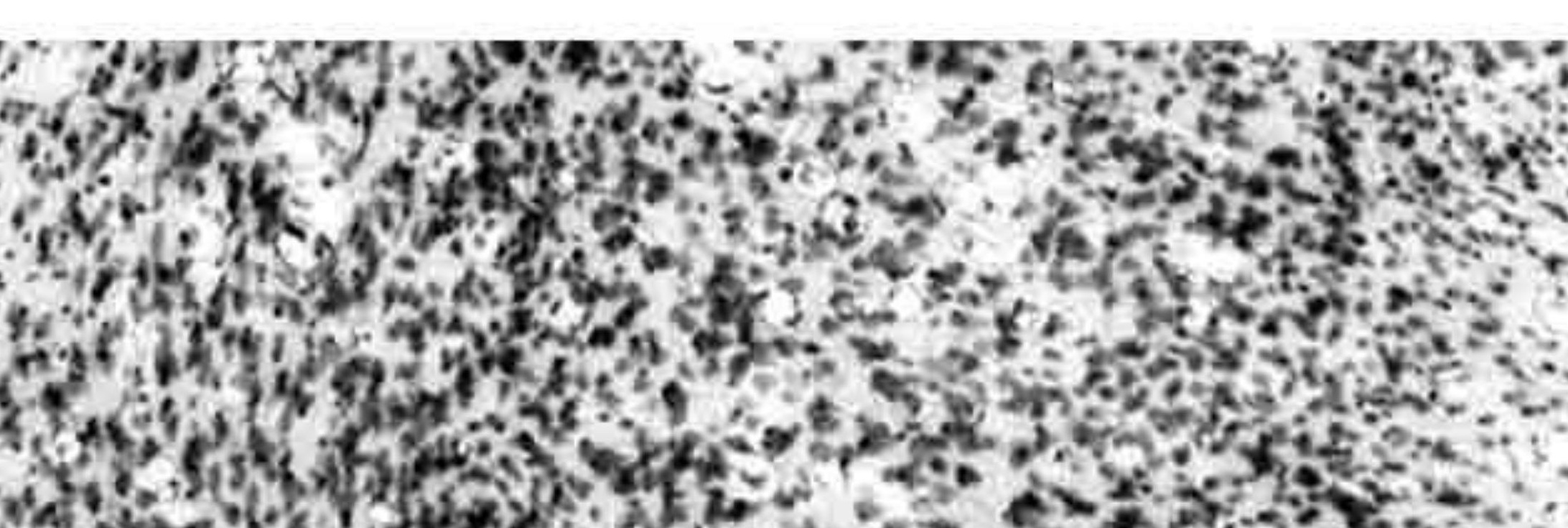

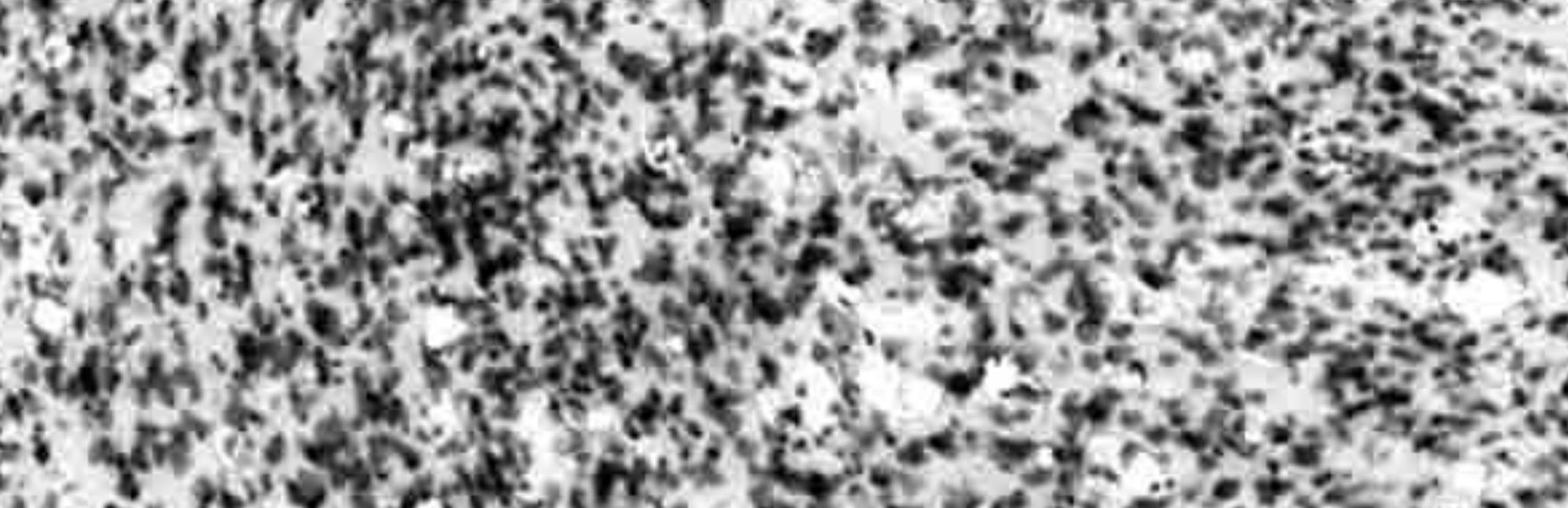

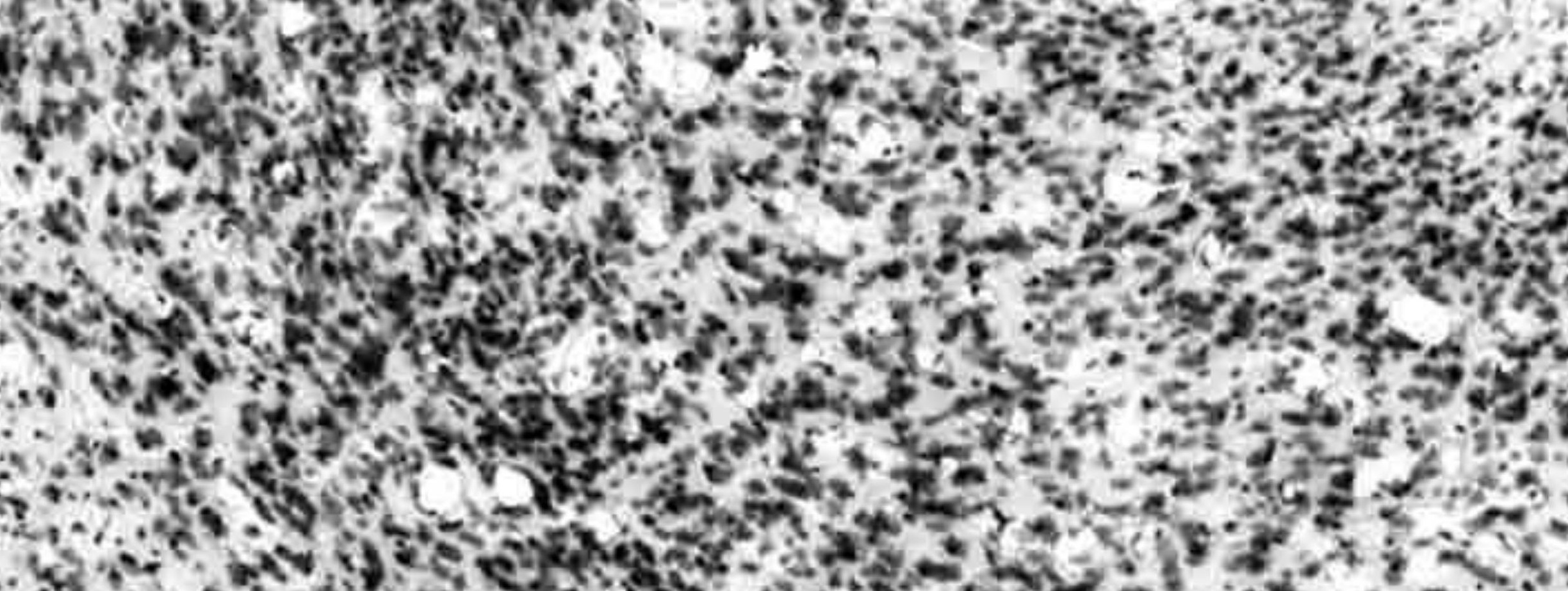

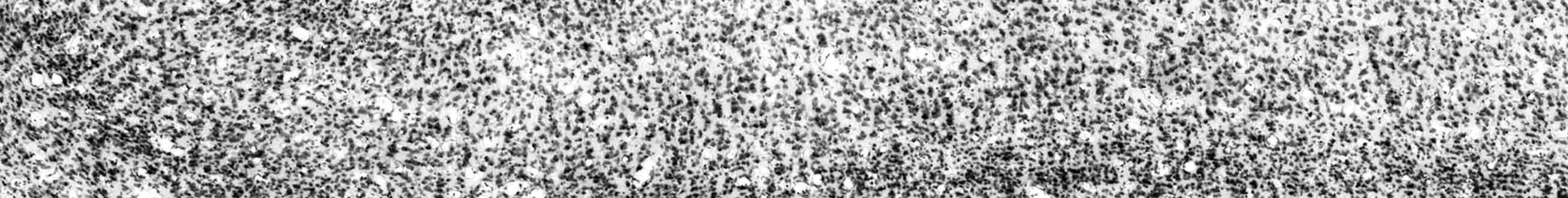

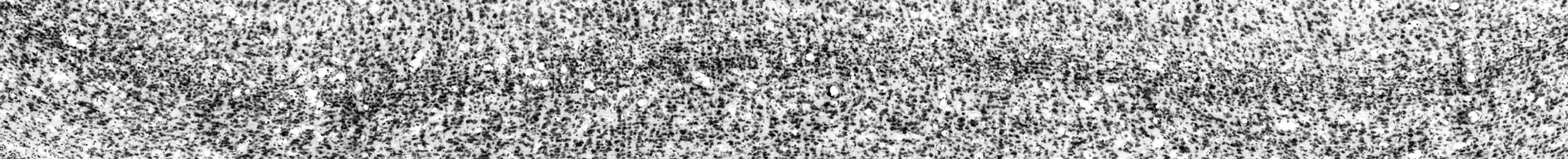

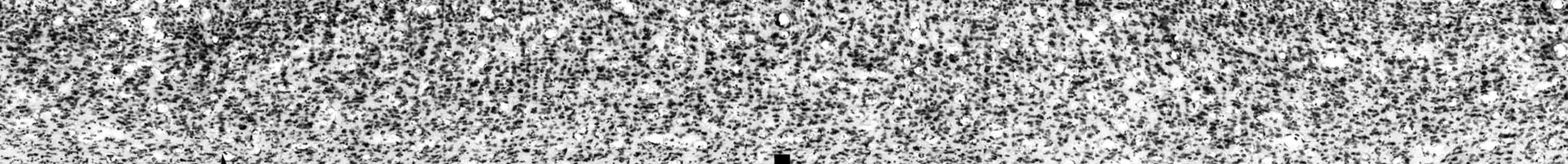

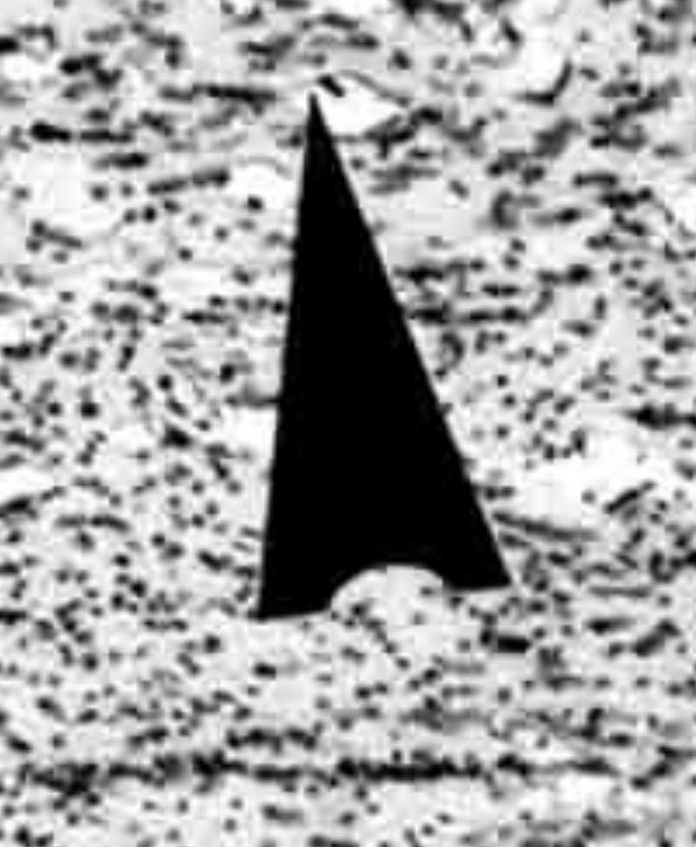

1

C

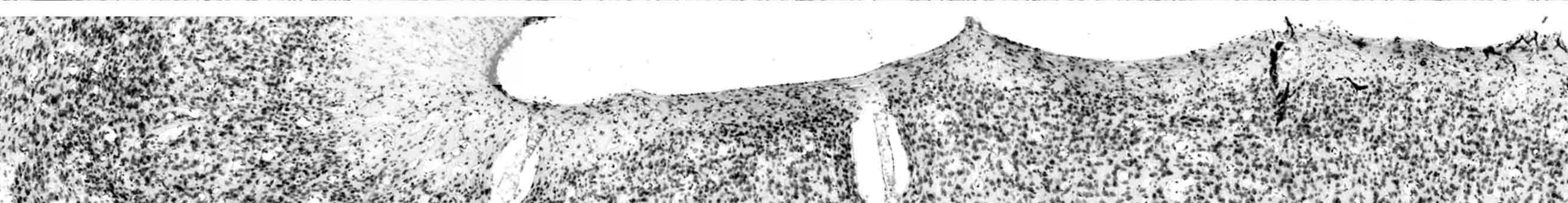

4.

\begin{tabular}{lll}
\hline \\
\hline
\end{tabular}

\section{$I d f p$}

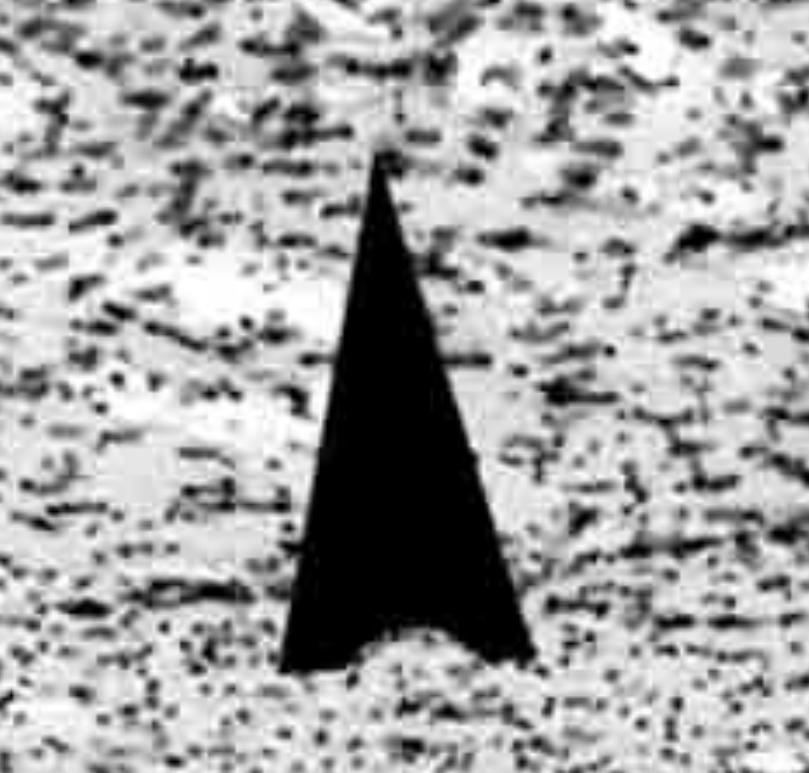

$$
I d d 2
$$

$I d d 4$

D
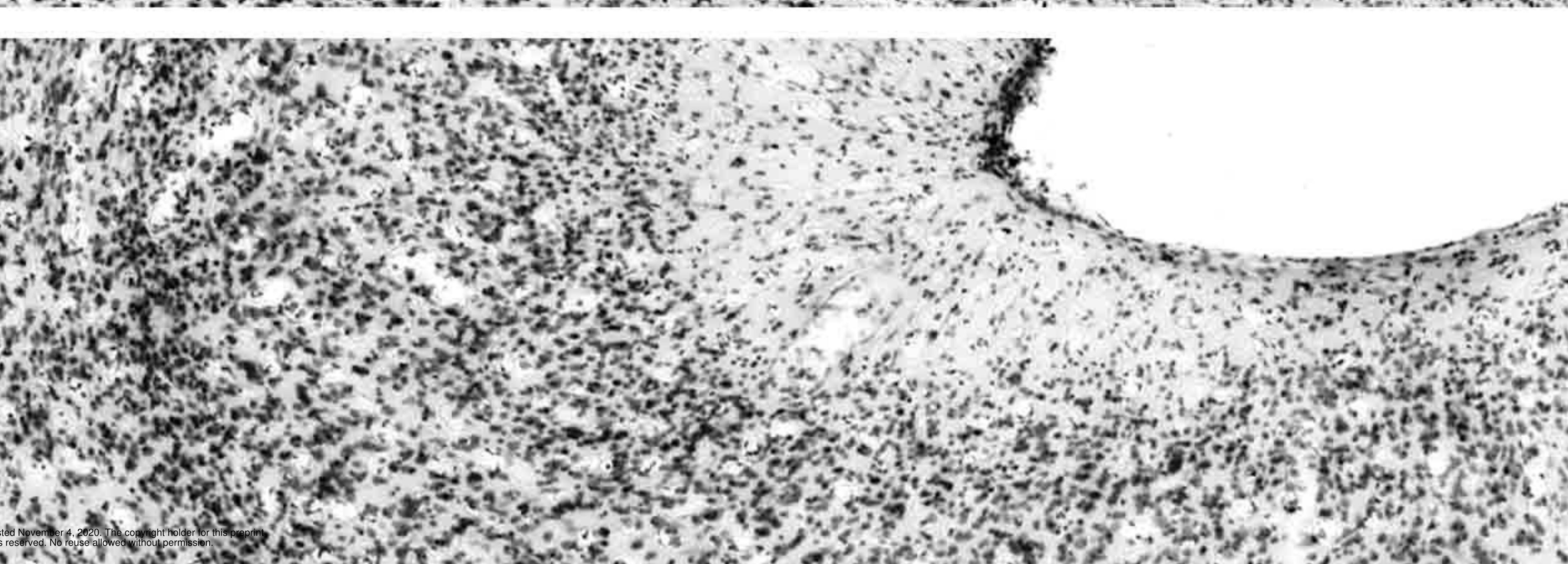

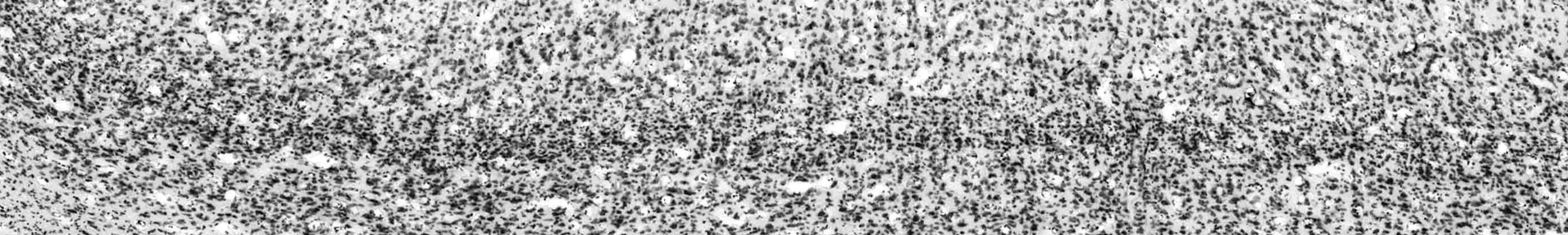

3.x. H.

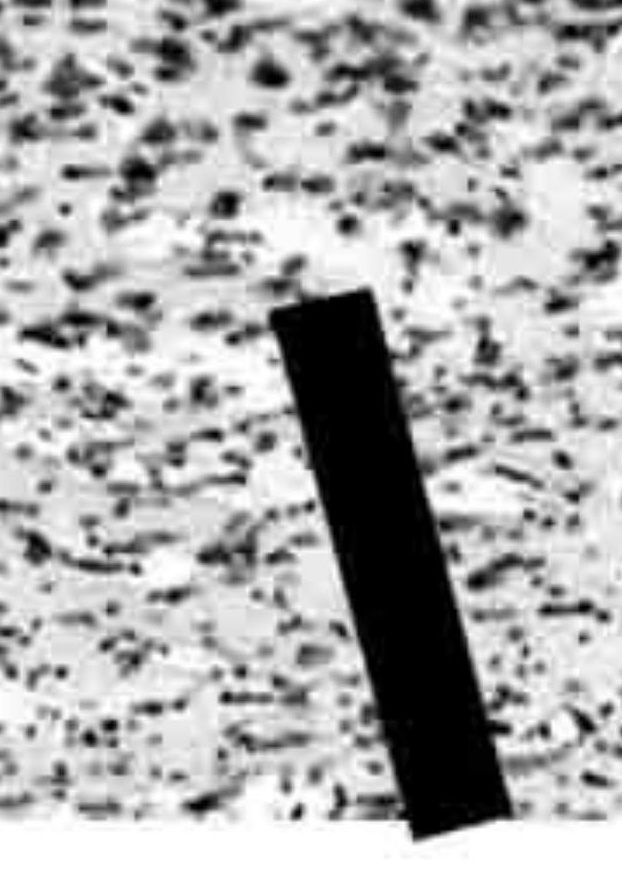

1

tro

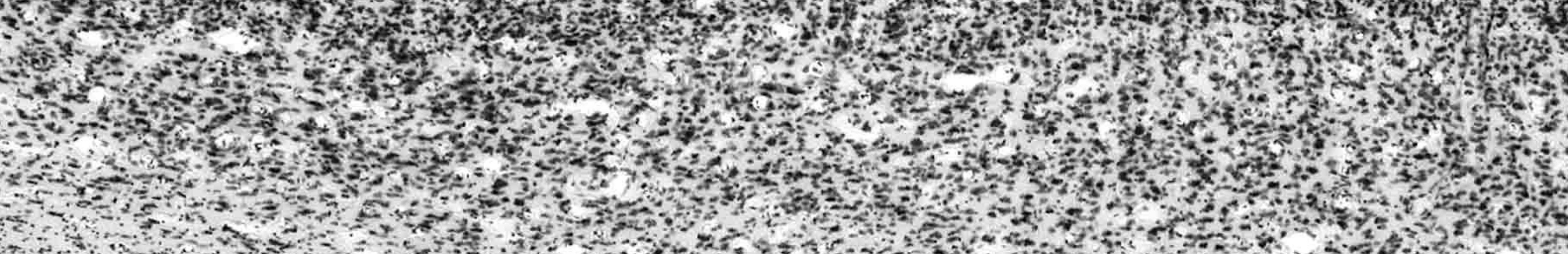

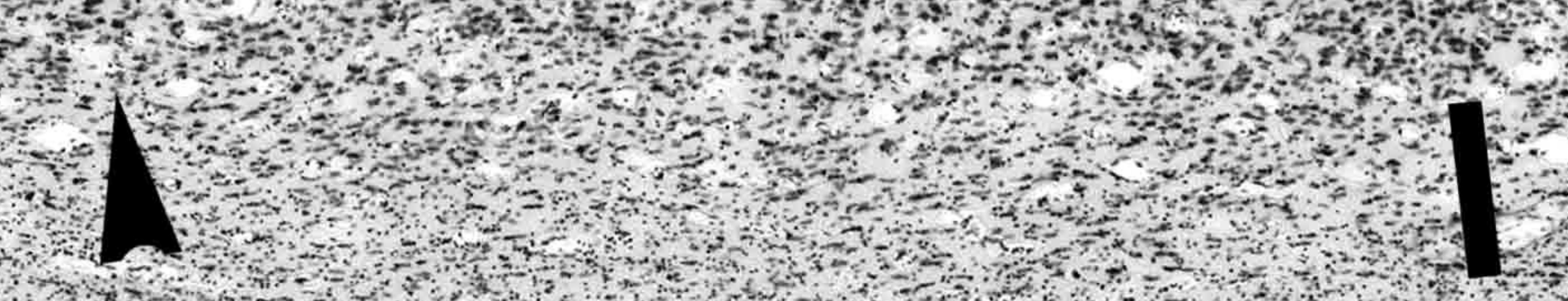

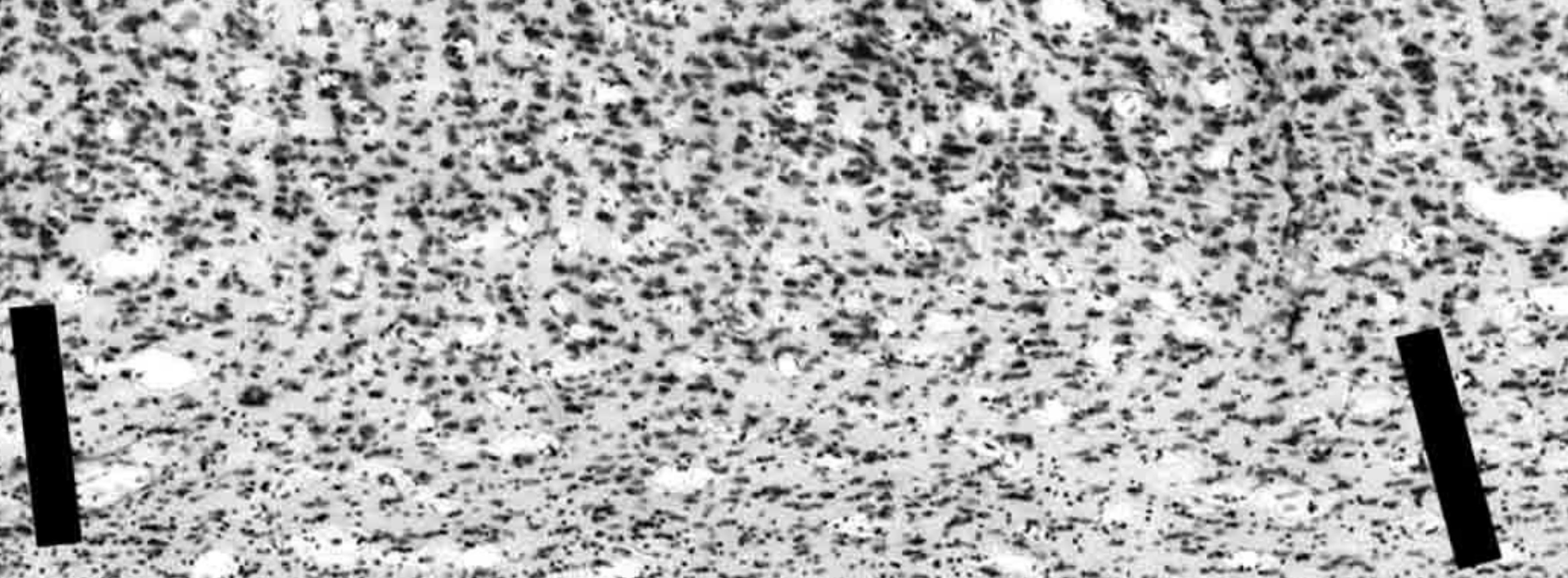




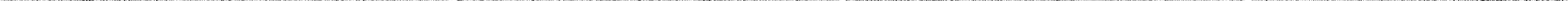




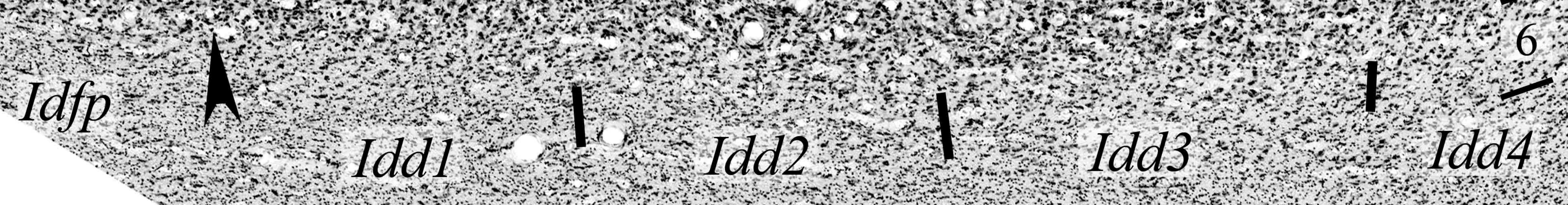

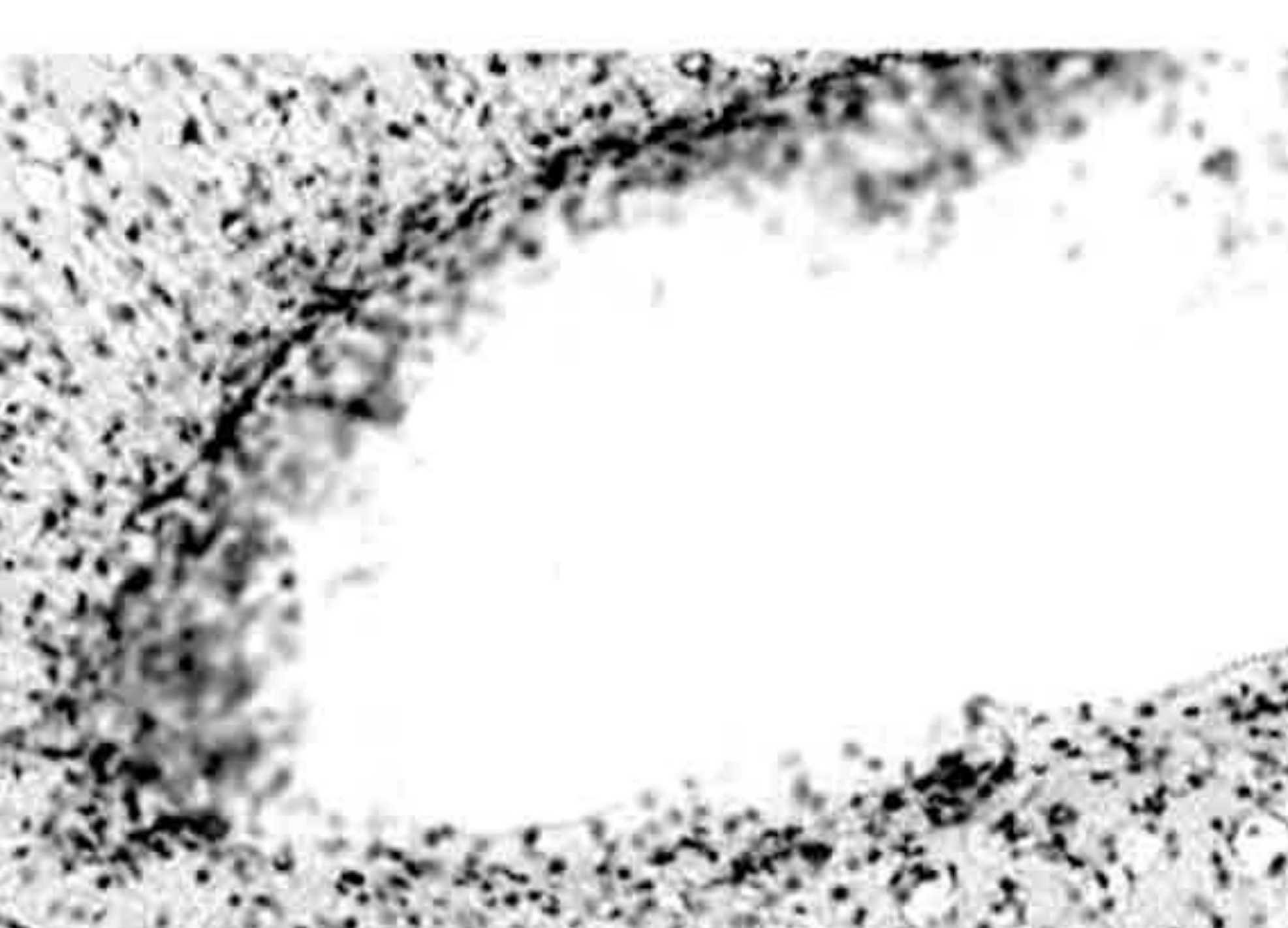

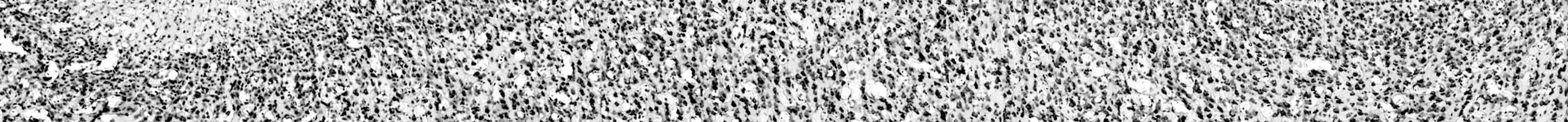

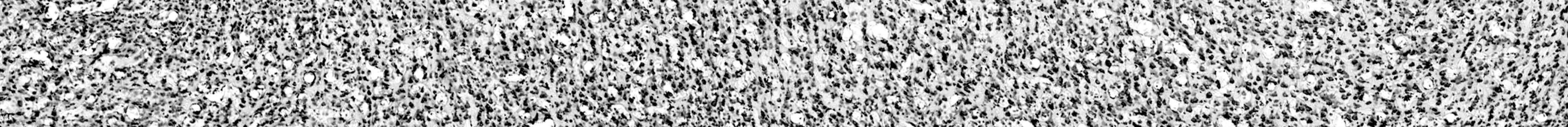

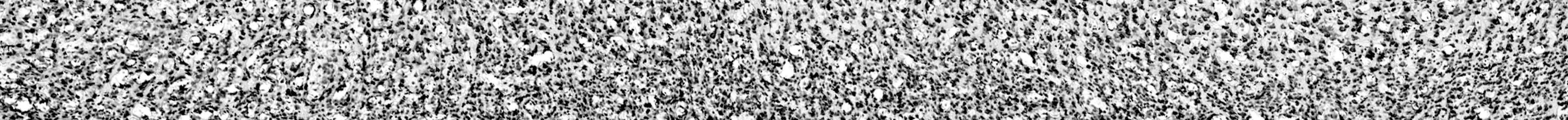

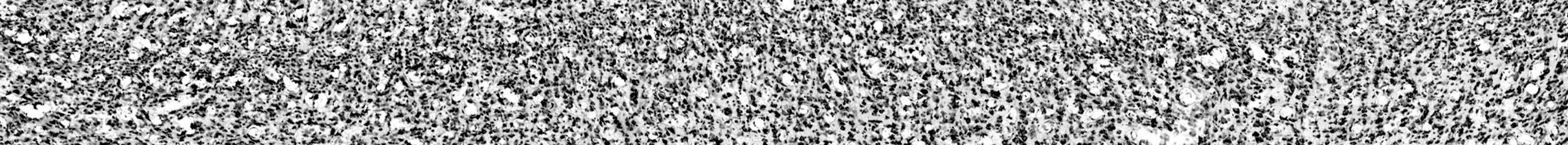

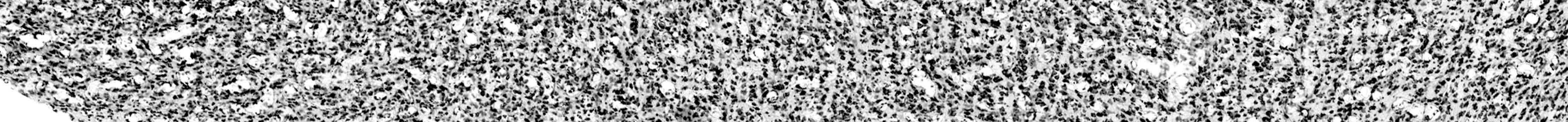
$I d f p$

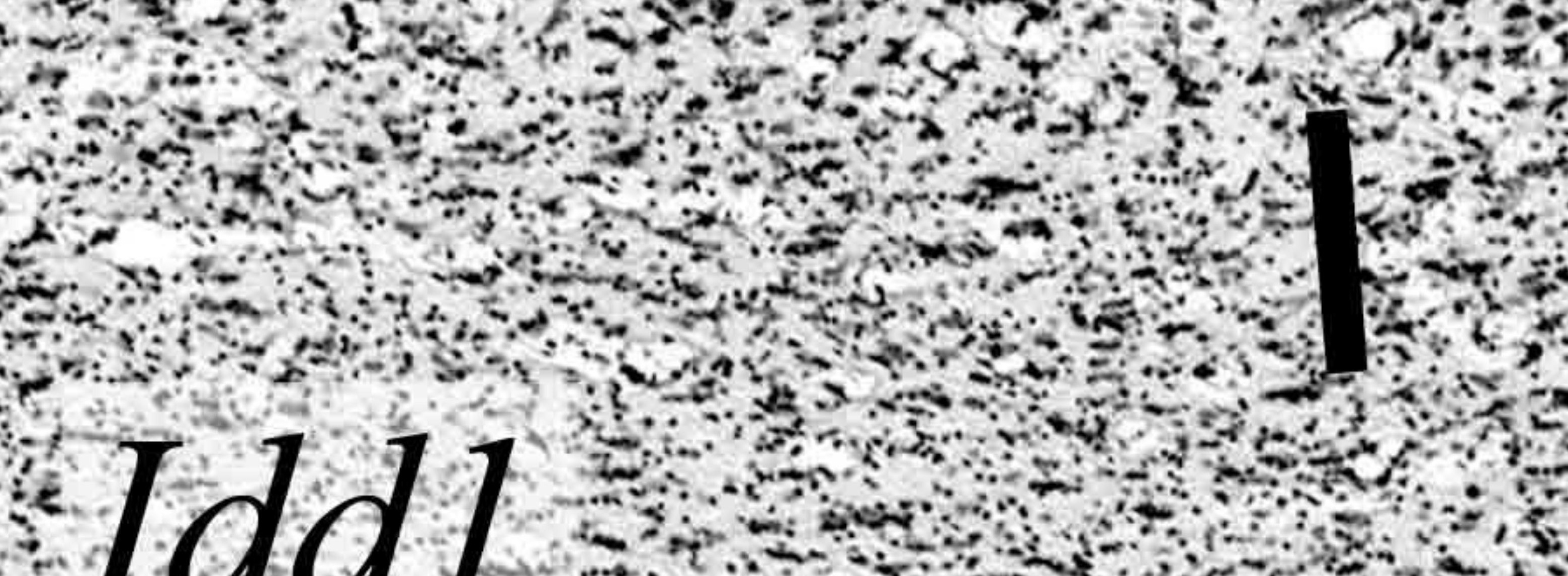
1.

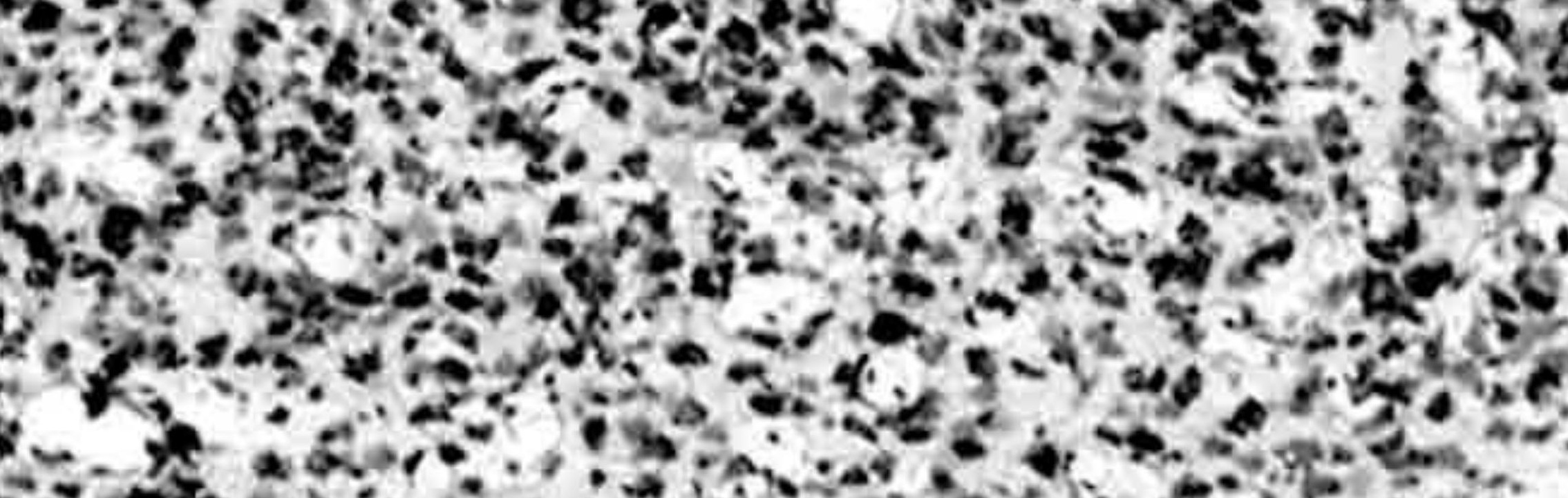

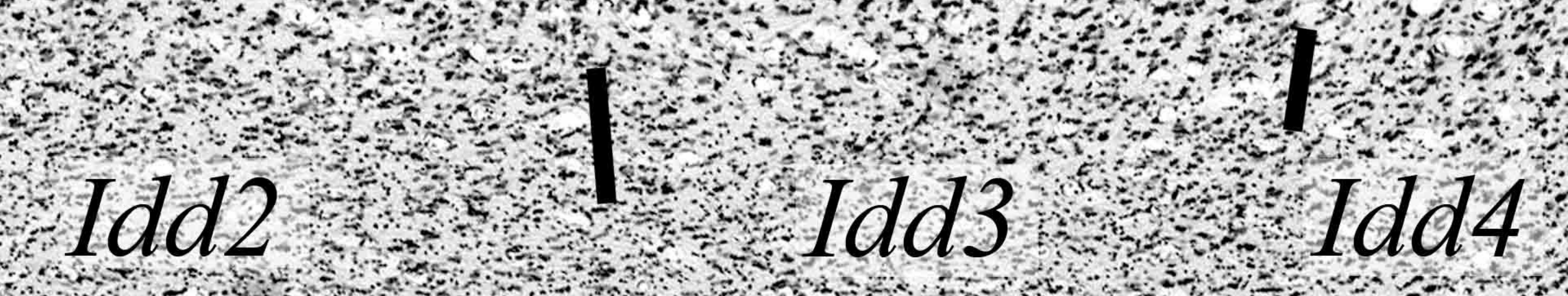

C

(2.2.

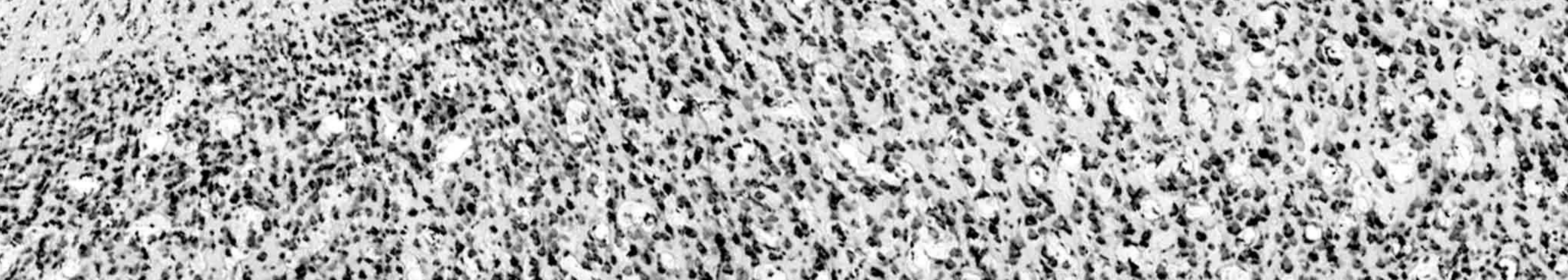

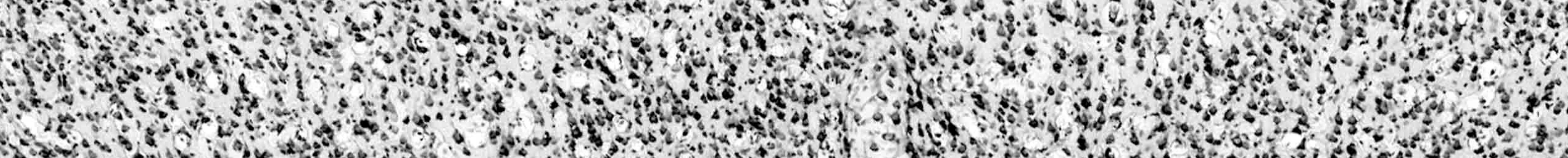

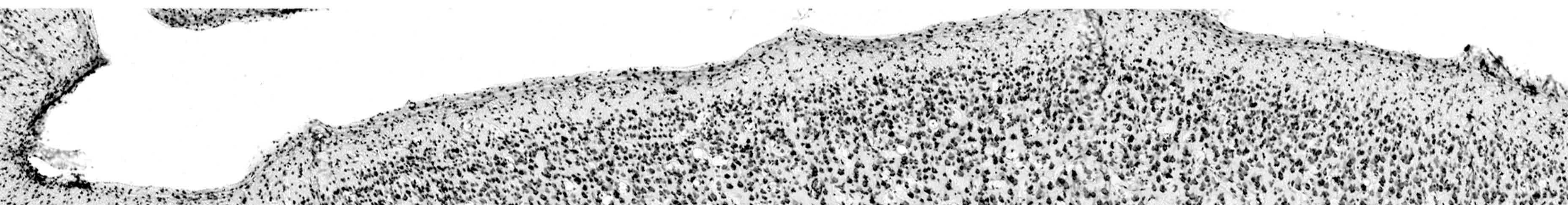



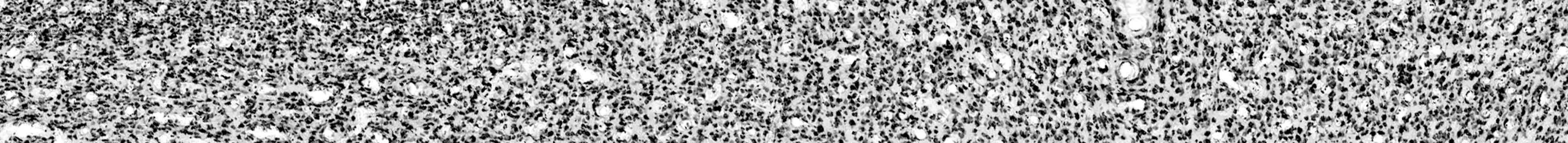

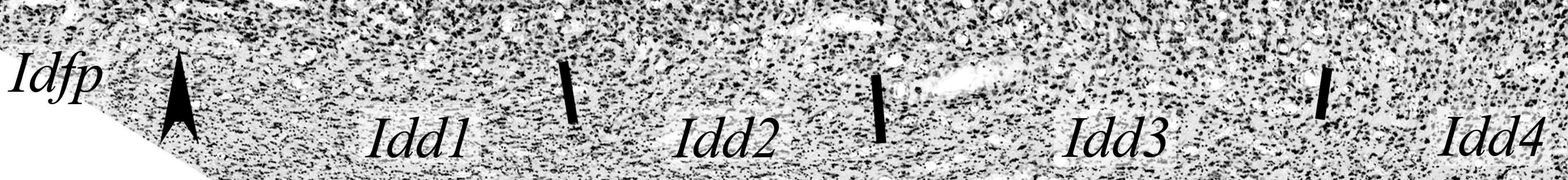




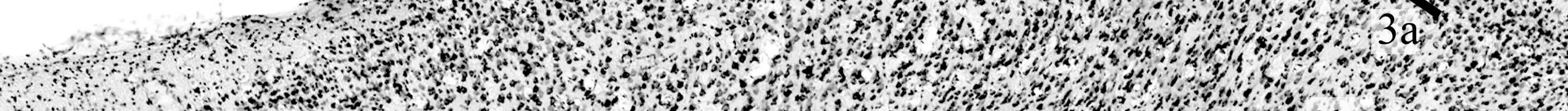

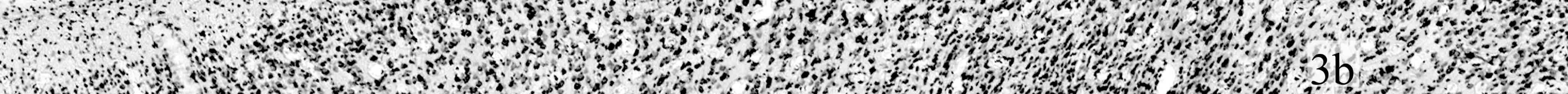

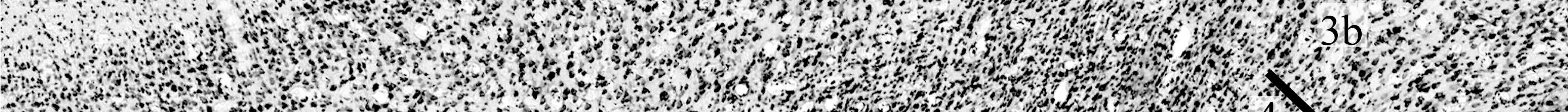

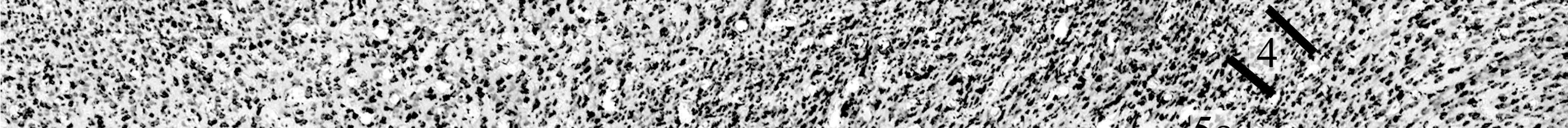

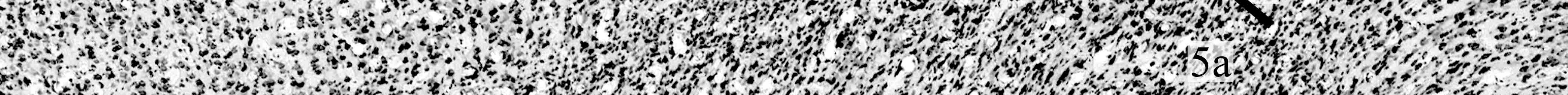
4.

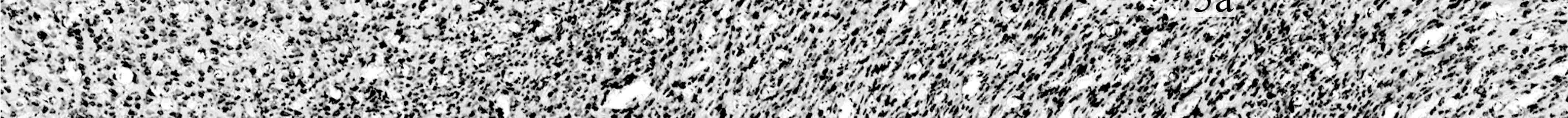
(3)

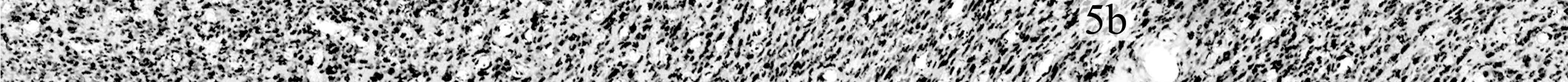
(3)

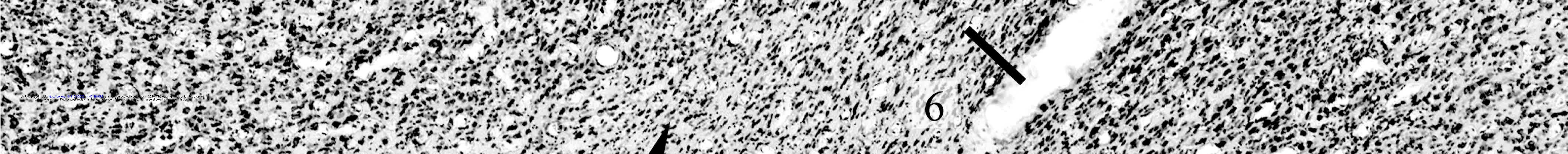

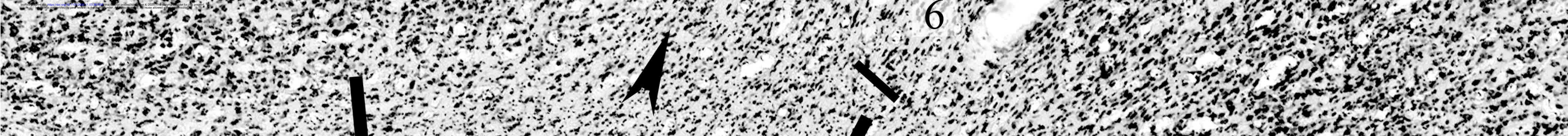

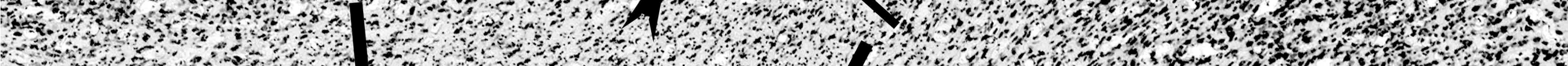

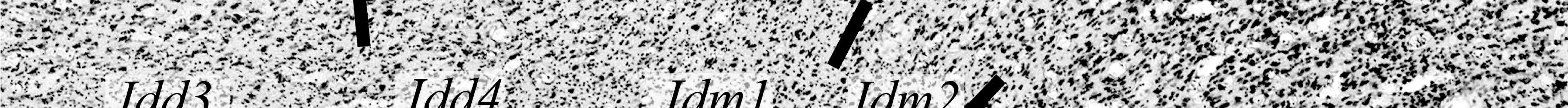

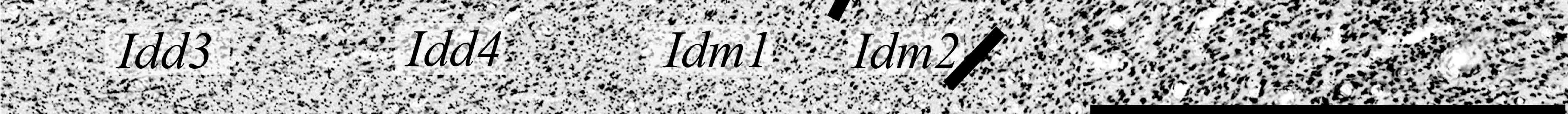




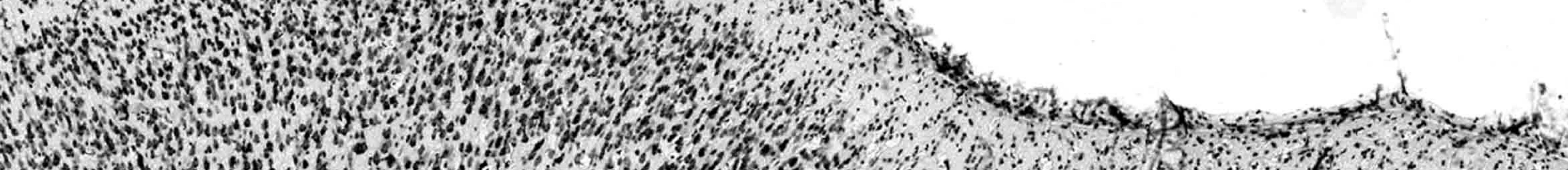

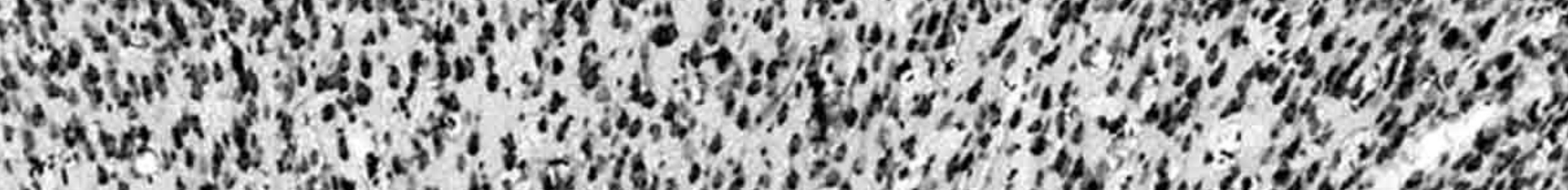

$\begin{aligned} 3 & \end{aligned}$

i.t.

Ning

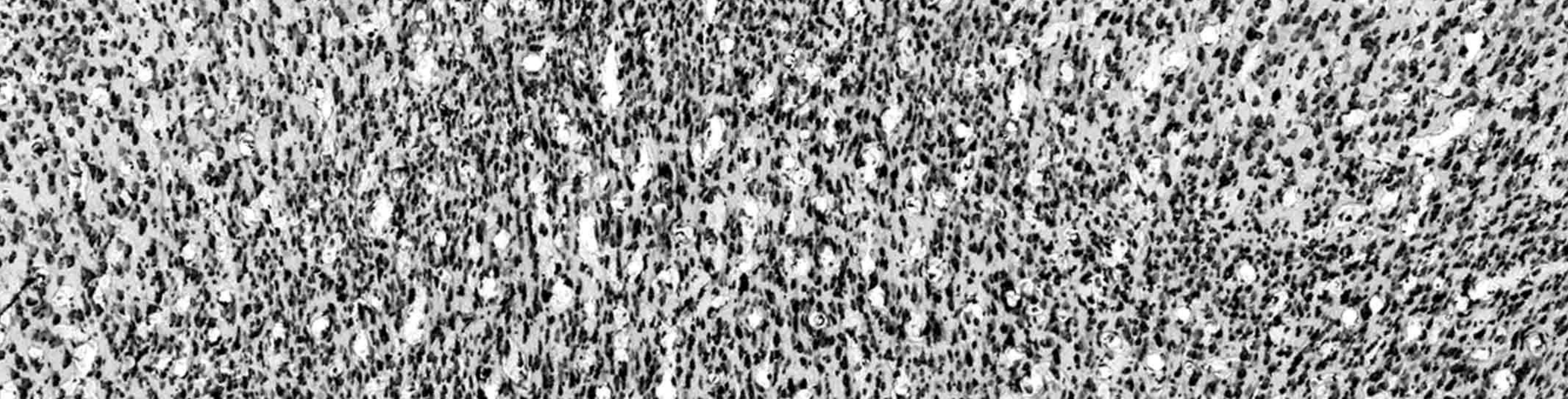

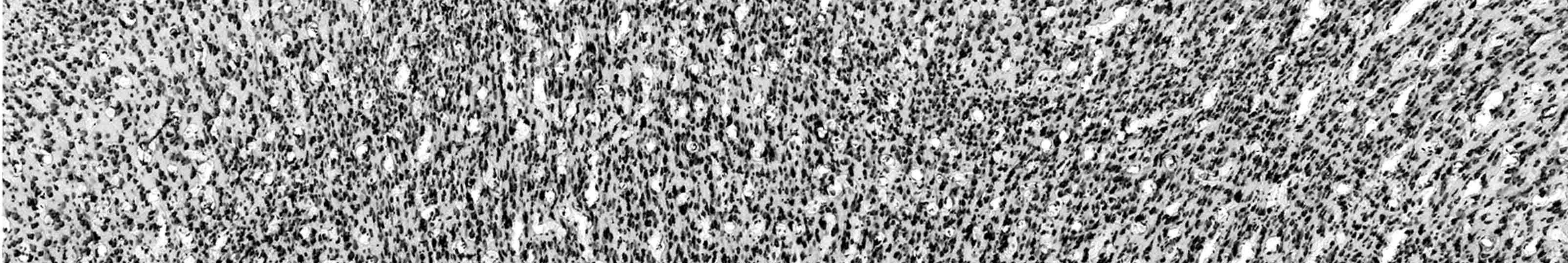



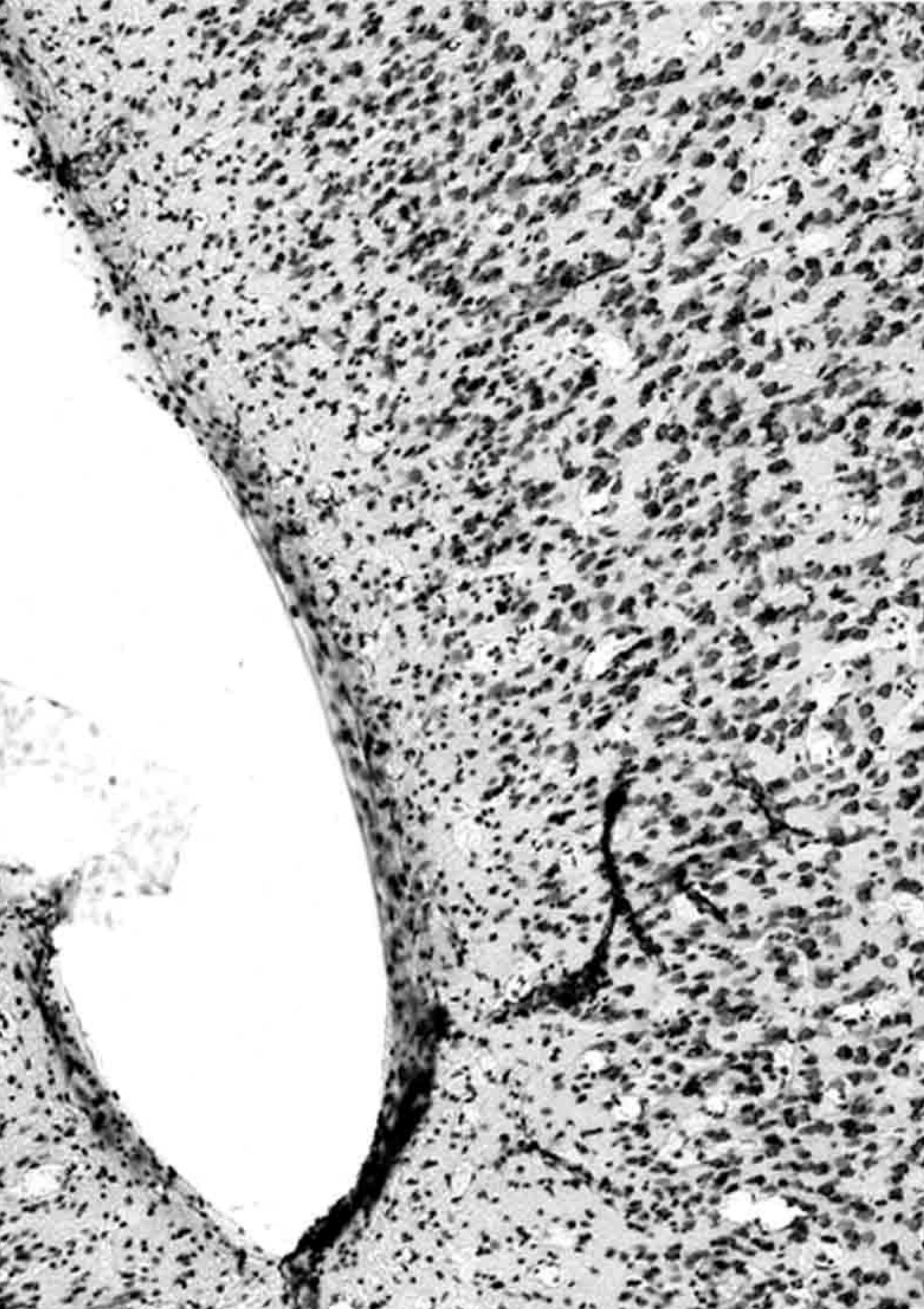

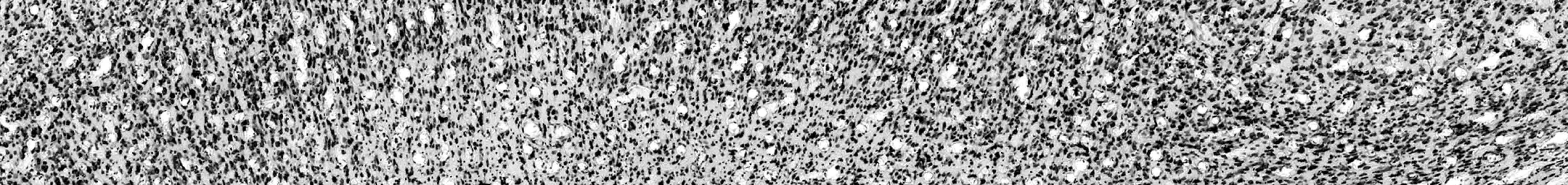

D
1dm 1. 1 Idm 3
Idd 5
Id 2

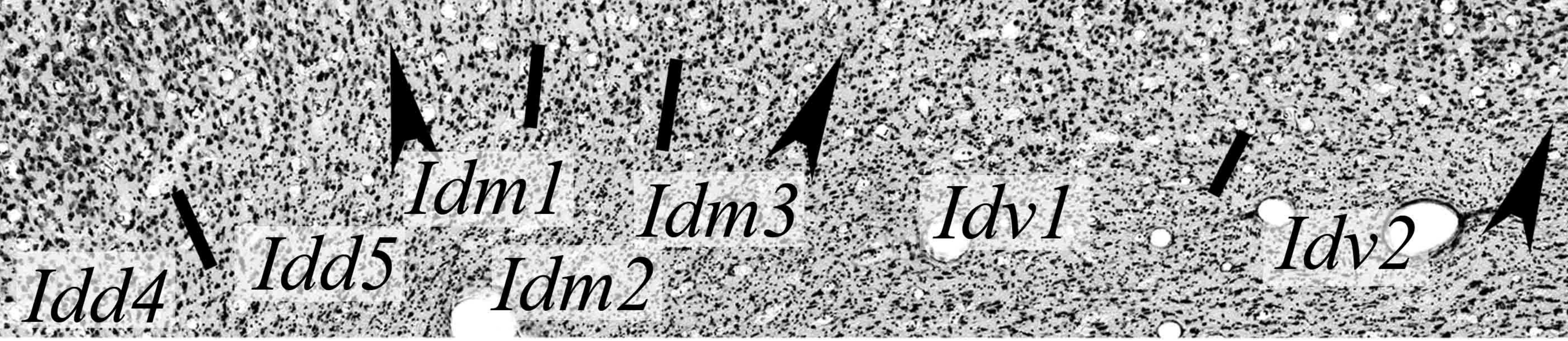


A

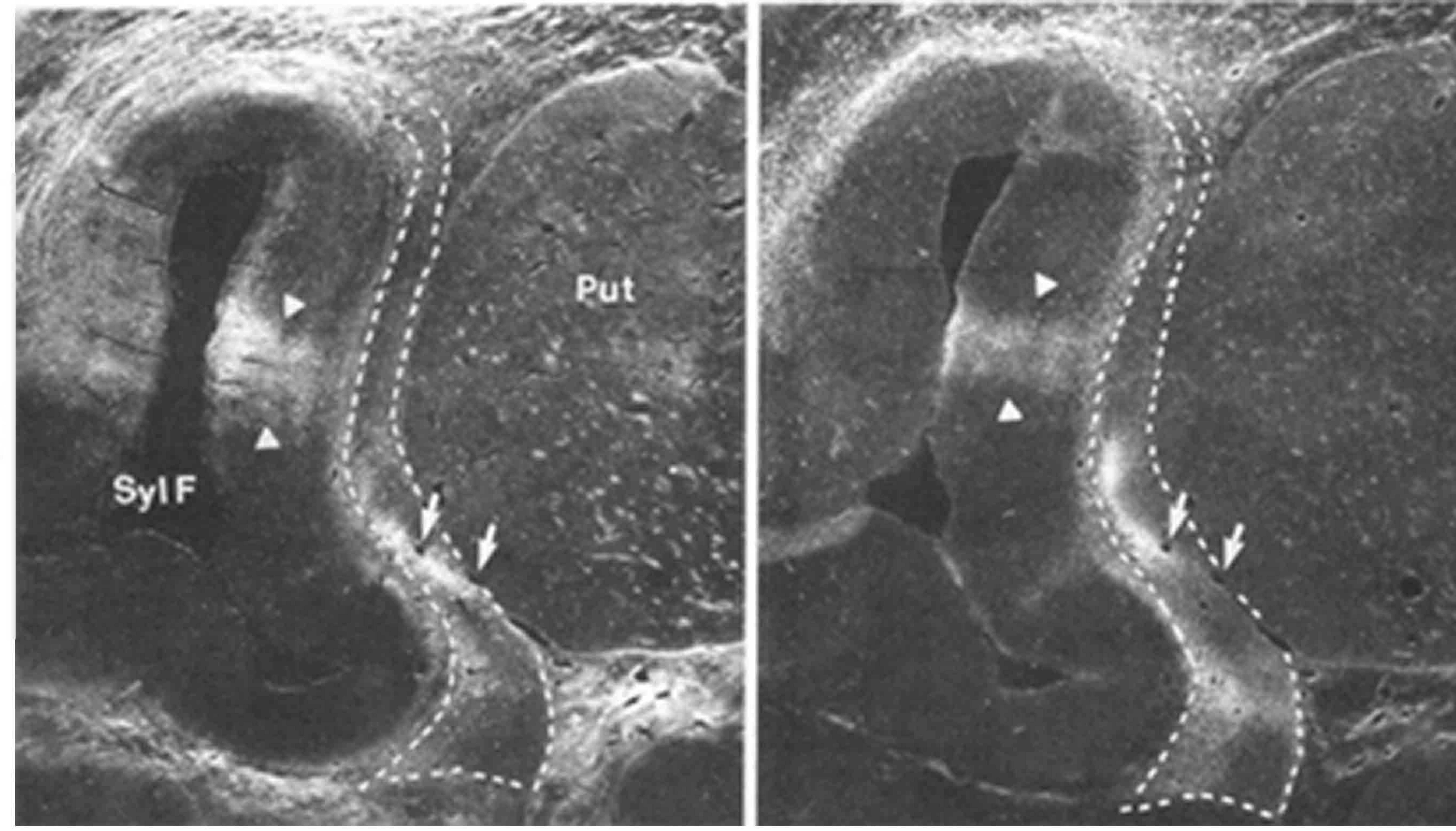

B

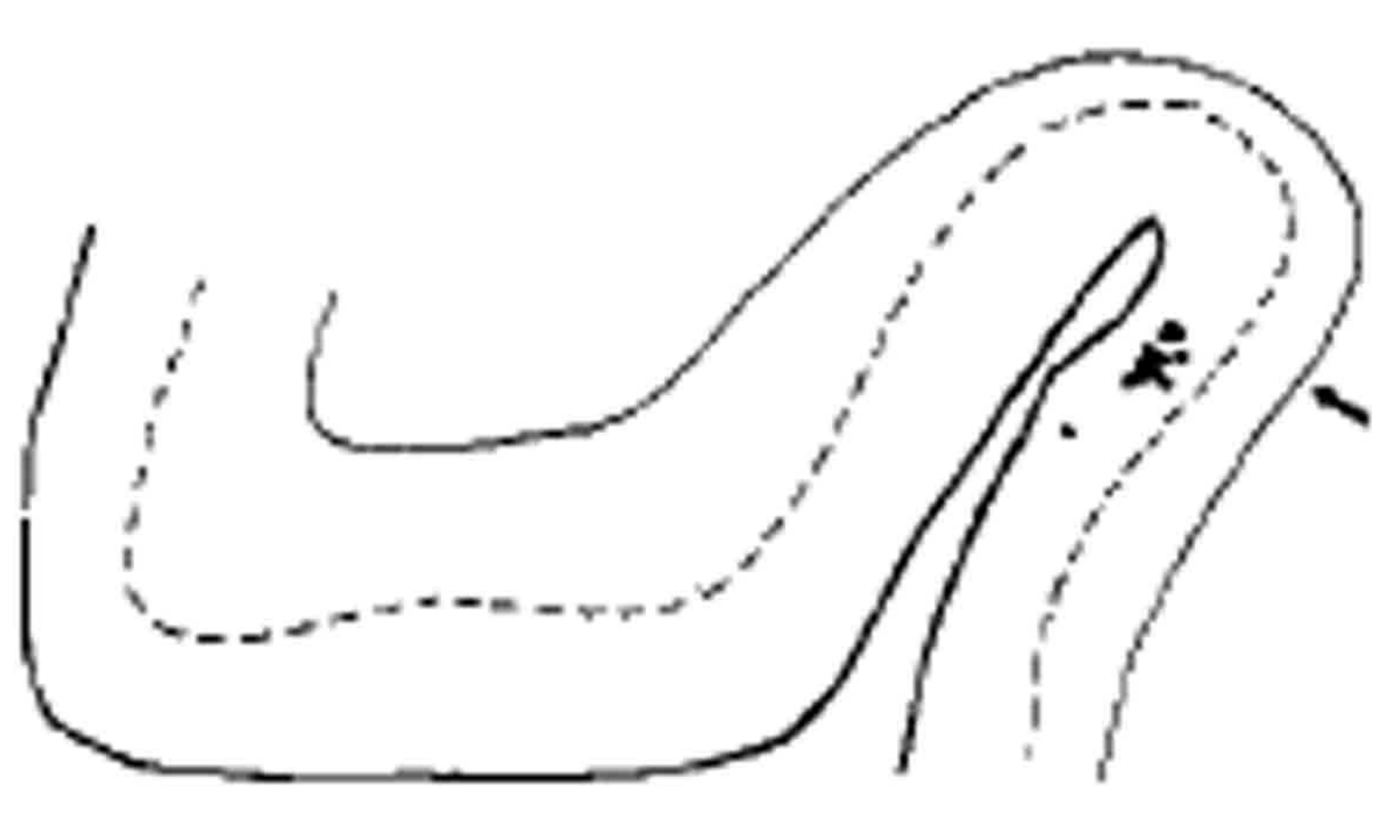

6800

C

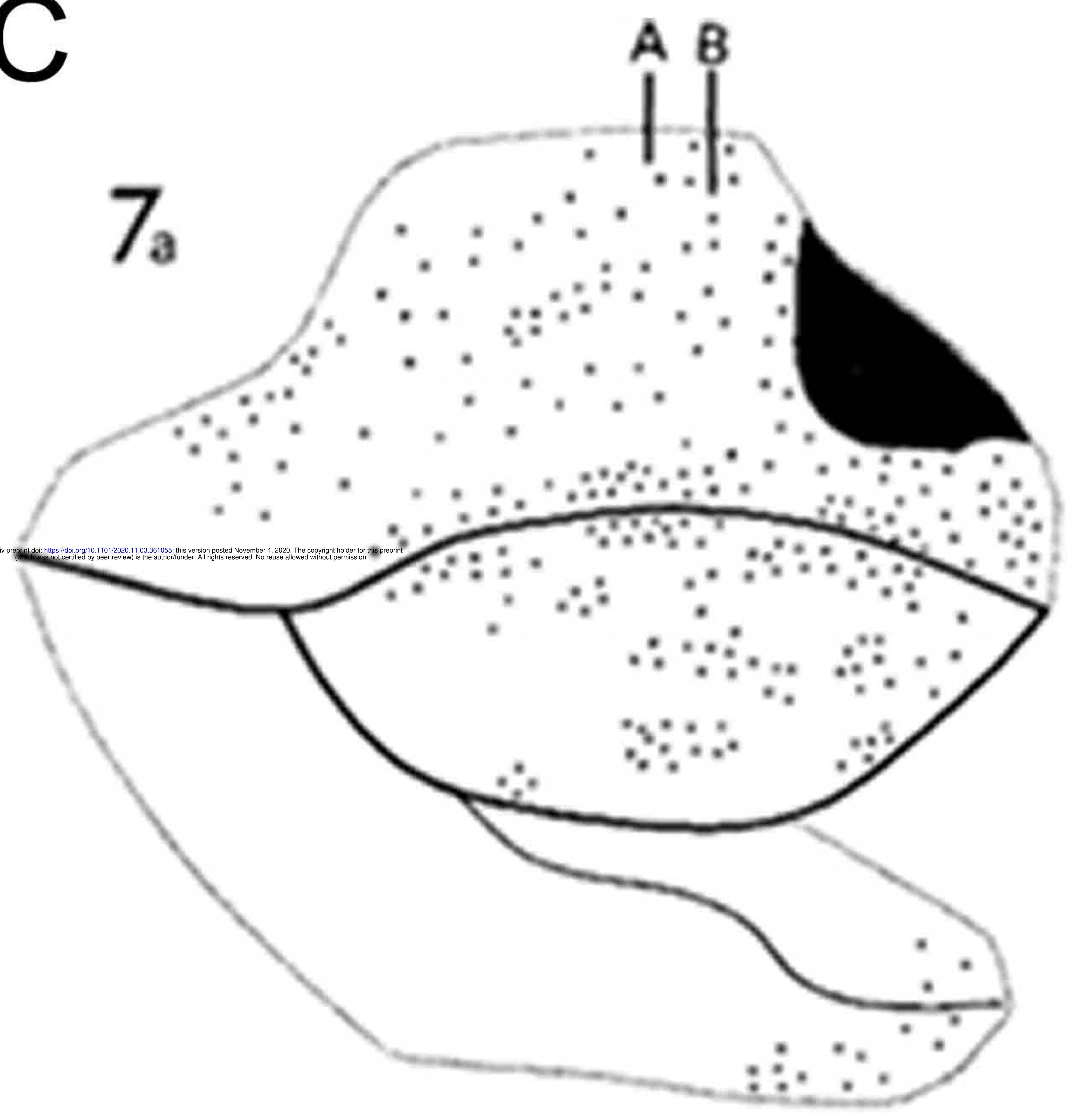

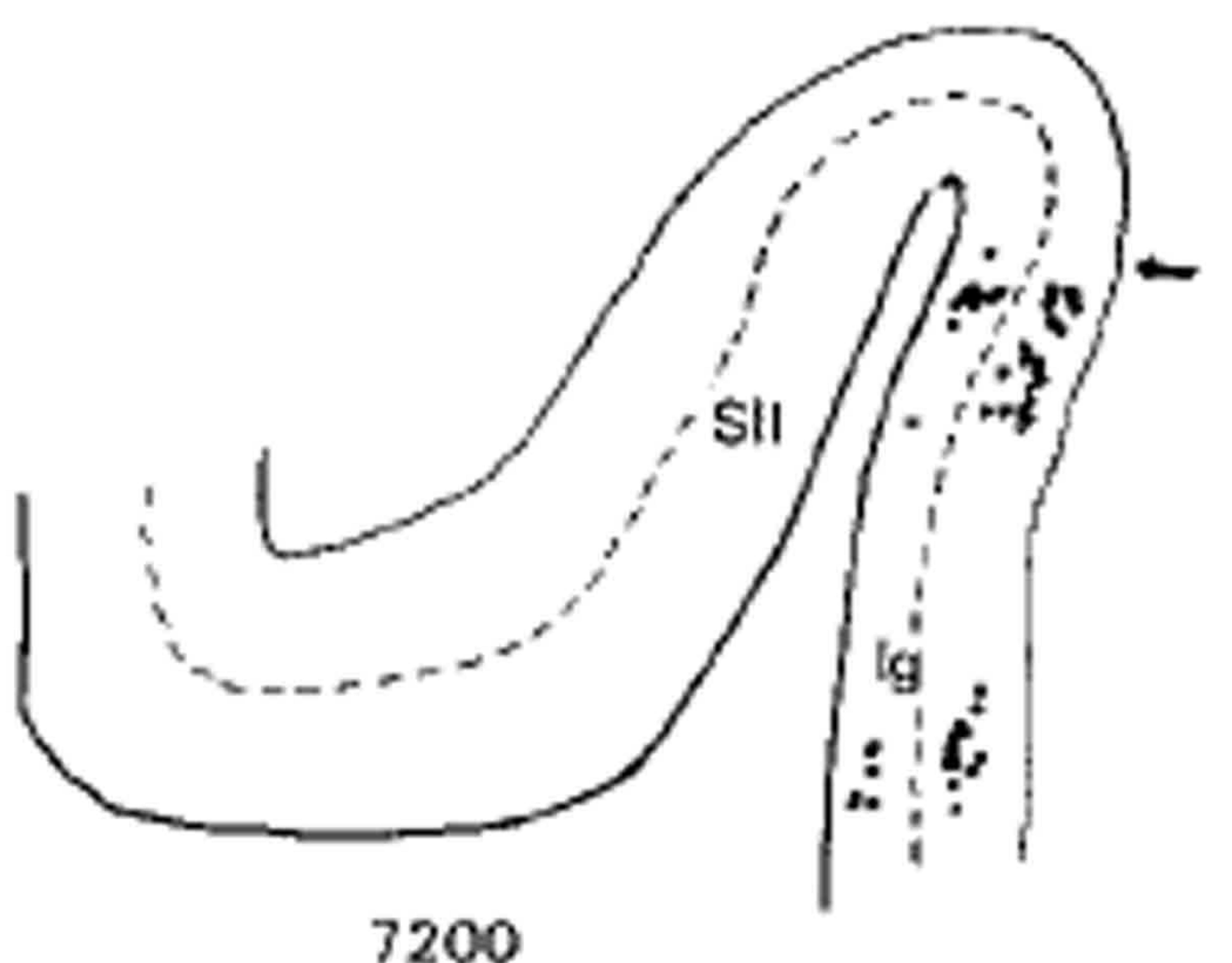

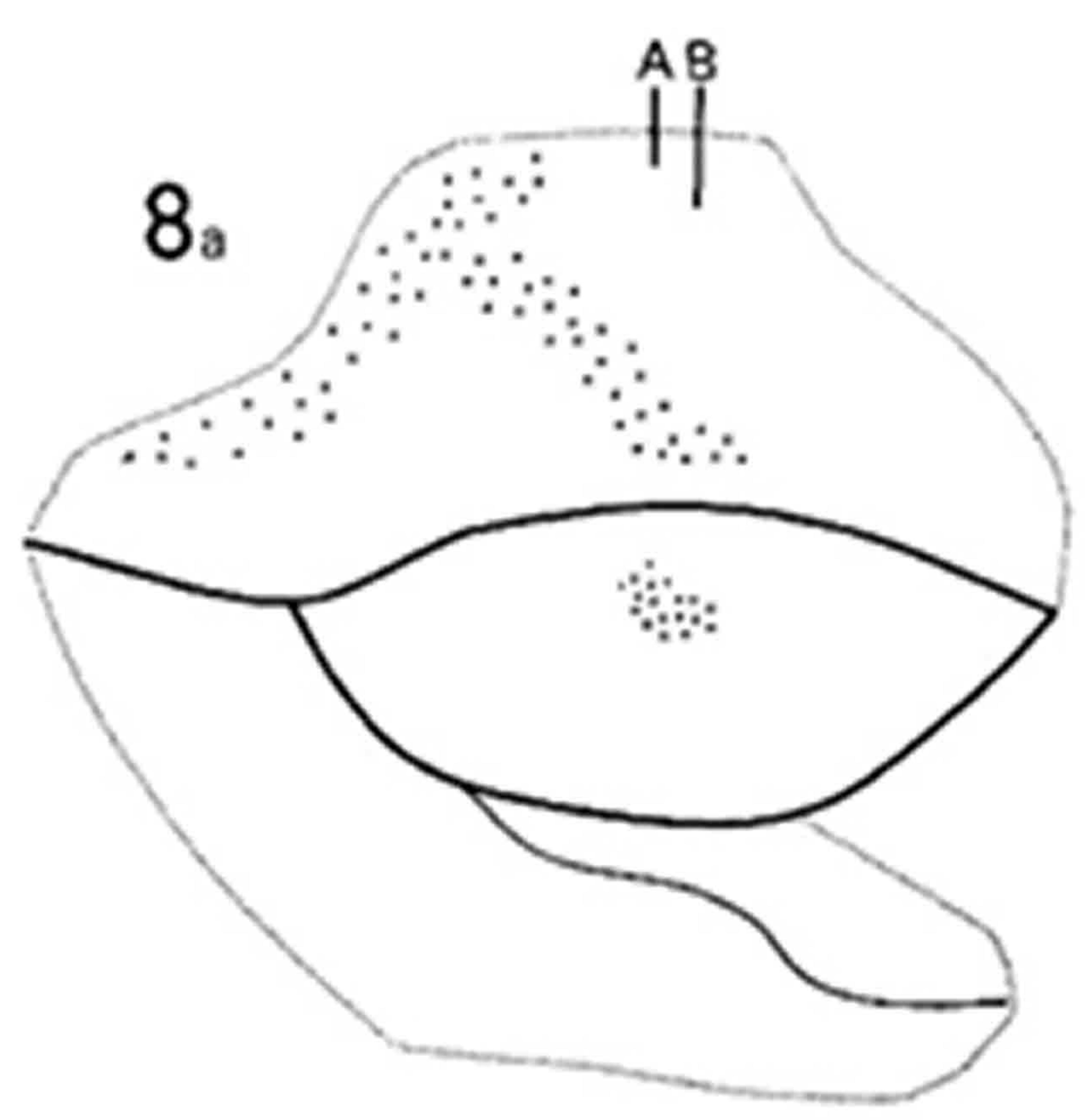

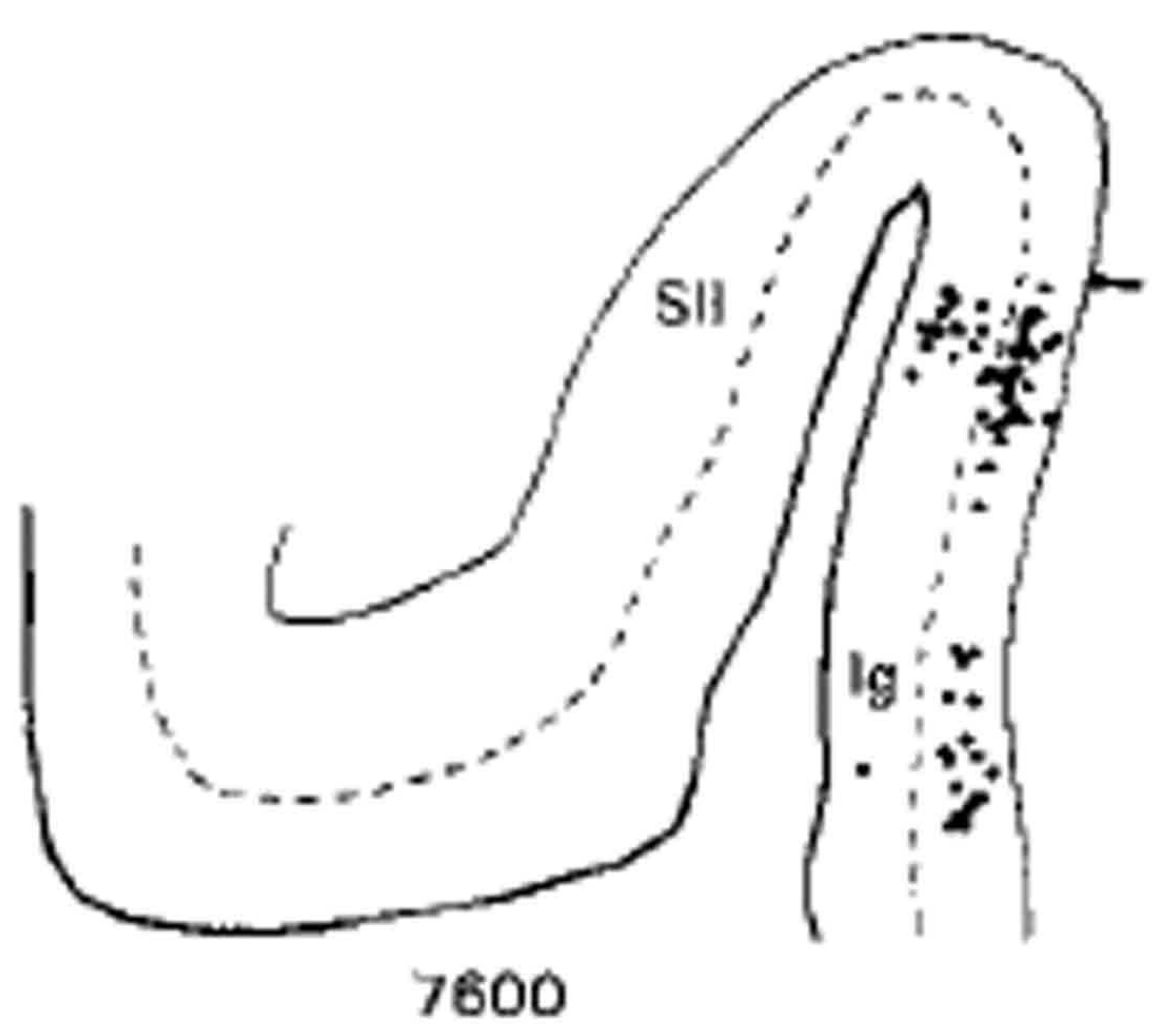



